\title{
PERIODIC ORBITS ABOUT AN OBLATE SPHEROID
}

\author{
BY \\ WILLIAM DUNCAN MACMILLAN
}

$\S 1$. Introduction.

The orbit of a particle about an oblate spheroid is not in general closed geometrically. The motion of the particle is not, therefore, in general, periodic from a geometric point of view. But if we consider the orbits as described by the particle in a revolving meridian plane which passes constantly through the particle several classes of closed orbits can be found in which the motion is periodic. The failure of these orbits to close in space arises from the incommensurability of the period of rotation of the line of nodes with the period of motion in the revolving plane. When these periods happen to be commensurable the orbits are closed in space and the motion is therefore periodic, though the period may be very great. Indeed, it seems that most of the difficulty in giving mathematical expressions for the orbits about an oblate spheroid rests upon the question of incommensurability of periods. The difficulty arising from the motion of the node can be overcome by the use of the revolving plane, but other incommensurabilities, such as that introduced by the eccentricity, can not be eliminated in this manner.

Orbits closed in the revolving plane are considered most conveniently in two general classes: (1) Those which reënter after one revolution, (2) those which reënter after many revolutions. The existence of both classes is established in this paper and convenient methods for constructing the solutions are given. Orbits which reënter after the first revolution are naturally the simpler and will be considered in the first part of the paper. Those lying in the equatorial plane of the spheroid become straight lines in the revolving plane, and it is shown that within the realm of convergence of the series employed all orbits in the equatorial plane are periodic. When the orbits do not lie in the equatorial plane there exists one, and only one, orbit for any arbitrarily assigned values of the inclination and the mean distance. These orbits reduce to circles with the vanishing of the oblateness of the spheroid.

In considering orbits which reënter only after many revolutions the differential equations are found to be very complex, and one would despair of proving the existence of these orbits by the ordinary direct computation of the necessary coefficients. However, a proof of their existence and a method for the construc- 
tion of the solutions are given by the aid of certain theorems, which are here established, on the character of the solutions of non-homogeneous linear differential equations with periodic coefficients.

These periodic orbits of many revolutions involve five constants, four of which are entirely arbitrary, and the fifth subject only to the limitation that it shall satisfy certain commensurability conditions. One constant, only, is missing for a complete integration of the differential equations. These orbits are all symmetric with respect to the equatorial plane.

\section{§ 2. The differential equations.}

The differential equations of motion of a particle about an oblate spheroid are *

$$
\begin{aligned}
& \frac{d^{2} x}{d t^{2}}=-\frac{k^{2} M x}{R^{3}}\left[1+\frac{3}{10} b^{2} \frac{x^{2}+y^{2}-4 z^{2}}{R^{4}} \mu^{2}+\cdots\right]=\frac{\partial V}{\partial x}, \\
& \frac{d^{2} y}{d t^{2}}=-\frac{k^{2} M y}{R^{3}}\left[1+\frac{3}{10} b^{2} \frac{x^{2}+y^{2}-4 z^{2}}{R^{4}} \mu^{2}+\cdots\right]=\frac{\partial V}{\partial y}, \\
& \frac{d^{2} z}{d t^{2}}=-\frac{k^{2} M z}{R^{3}}\left[1+\frac{3}{10} b^{2} \frac{3\left(x^{2}+y^{2}\right)-2 z^{2}}{R^{4}} \mu^{2}+\cdots\right]=\frac{\partial V}{\partial z} \text {. }
\end{aligned}
$$

The symbols employed are defined as follows: $x, y, z$ are rectangular coördinates, the origin being at the center of the spheroid and the $x y$-plane the plane of the equator, $k$ is the Gaussian constant, $M$ is the mass of the spheroid, $b$ is the polar radius of the spheroid, $\mu$ is the eccentricity of the spheroid,

Since

$$
R=\sqrt{x^{2}+y^{2}+z^{2}}, \quad V=\frac{k^{2} M}{R}\left[1+\frac{b^{2}}{10} \frac{x^{2}+y^{2}-2 z^{2}}{R^{4}} \mu^{2}+\cdots\right] .
$$

$$
\frac{1}{x} \frac{\partial V}{\partial x}=\frac{1}{y} \frac{\partial V}{\partial y},
$$

we obtain one integral of areas, namely,

$$
x \frac{d y}{d t}-y \frac{d x}{d t}=c_{1} \text {. }
$$

That is, the projection of the area described by the radius vector upon the equa: torial plane is proportional to the time. We have also the vis viva integral

$$
\left(\frac{d x}{d t}\right)^{2}+\left(\begin{array}{c}
d y \\
d t
\end{array}\right)^{2}+\left(\frac{d z}{d t}\right)^{2}=2 V+c_{2}
$$

For further integration we are compelled to resort to series. Poincaré has shown in his Les méthodes nouvelles de la mécanique céleste that if certain

\footnotetext{
* Moulton, Celestial Mechanics, p. 113.
} 
conditions are fulfilled it is possible to obtain periodic orbits represented by power series in a parameter when periodic orbits are known for zero values of this parameter. These conditions are at least partially fulfilled in the present problem, for the right members of the differential equations are analytic in $x, y, z$ and the parameter $\mu$. Furthermore, for $\mu=0$, the equations reduce to the ordinary two-body problem for which periodic solutions are known. It is our purpose to show that the remaining necessary conditions also are fulfilled and that periodic solutions persist for values of $\mu \neq 0$. These periodic series are very satisfactory, for the general character of the orbits represented by them is easily obtained. The solutions thus found are rigorous, but they are not general, their existence depending upon special initial conditions.

It will be advantageous to transform the differential equations to cylindrical coördinates by the substitutions

$$
\begin{aligned}
x & =a r \cos v, & k^{2} M & =n^{2} a^{3}, \\
y & =a r \sin v, & c_{1} & =c k \sqrt{\overline{M a},} \\
z & =a q, & u t & =\tau, \\
R & =a \sqrt{r^{2}+q^{2},} & \frac{3}{10} \frac{b^{2}}{a^{2}} & =\theta_{1}^{2} .
\end{aligned}
$$

After these substitutions equations (1) become

$$
\begin{array}{rlrl}
\text { (a) } r & r^{\prime \prime}-r v^{\prime 2} & =\frac{-r}{\left(r^{2}+q^{2}\right)^{\frac{1}{2}}}\left[1+\frac{r^{2}-4 q^{2}}{\left(r^{2}+q^{2}\right)^{2}} \theta_{1}^{2} \mu^{2}+\cdots\right], \\
\text { (b) } r v^{\prime \prime}+2 r^{\prime} v^{\prime} & =0, \\
\text { (c) } r & q^{\prime \prime} & =\frac{-q}{\left(r^{2}+q^{2}\right)^{\frac{1}{3}}}\left[1+\frac{3 r^{2}-2 q^{2}}{\left(r^{2}+q^{2}\right)^{2}} \theta_{1}^{2} \mu^{2}+\cdots\right],
\end{array}
$$

the accents denoting the derivatives with respect to $\tau$.

The integral of $(b)$ is $r^{2} v^{\prime}=c$, by means of which $v^{\prime}$ can be eliminated from the first of these equations. After the elimination the equations take the form

$$
r^{\prime \prime}=\frac{c^{2}}{r^{3}}-\frac{r}{\left(r^{2}+q^{2}\right)^{\frac{3}{3}}}-\frac{r^{3}-4 r q^{2}}{\left(r^{2}+q^{2}\right)^{\frac{3}{3}}} \theta_{1}^{2} \mu^{2}+\cdots,
$$

$$
q^{\prime \prime}=-\frac{q}{\left(r^{2}+q^{2}\right)^{\frac{3}{2}}}-\frac{3 r^{2} q-q^{3}}{\left(r^{2}+q^{2}\right)^{\frac{2}{2}}} \theta_{1}^{2} \mu^{2}+\cdots,
$$

(c) $\quad v^{\prime}=\frac{c}{r^{2}}$.

The first two of these equations are indenendent of the third so that $r$ and $q$ may be considered as rectangular coördinates in a revolving plane which passes 
always through the $z$-axis and through the particle itself. The problem is thus reduced to the consideration of the motion in this plane, for, when $r$ is known, $v$ is obtained by a simple quadrature from (6c).

\section{§ 3. Surfaces of zero velocity.}

The velocity integral in the revolving plane is

$$
r^{\prime 2}+q^{\prime 2}=\frac{2}{\left(r^{2}+q^{2}\right)^{\frac{1}{2}}}+\frac{2}{3} \frac{r^{2}-2 q^{2}}{\left(r^{2}+q^{2}\right)^{\frac{5}{2}}} \theta_{1}^{2} \mu^{2}+\cdots-\frac{c^{2}}{r^{2}}+c_{2} .
$$

If we put the velocity equal to zero the resulting equation represents a twoparameter family of curves. For assigned values of the parameters $c$ and $c_{2}$ there is defined a certain curve in the revolving plane. On one side of this curve the motion is real while on the other side it is imaginary. For values of $c_{2}<0$ this curve is closed, and the motion is real on the inside. As the plane revolves this curve generates a surface of the general form of an anchor ring. For $\mu^{2}=0$ this curve belongs to the ordinary two-body problem, and its equation is

$$
\frac{2}{\left(r^{2}+q^{2}\right)^{\frac{3}{2}}}-\frac{c^{2}}{r^{2}}+c_{2}=0 \text {. }
$$

The motion is elliptic, parabolic, or hyperbolic according as $c_{2}$ is negative, zero, or positive. Putting

we find, on solving for $\rho$,

$$
r=\rho \cos \phi, \quad q=\rho \sin \phi,
$$

$$
\rho=\frac{1}{c_{2}}\left[-1 \pm \sqrt{1+\frac{c_{2} c^{2}}{\cos ^{2} \phi}}\right] .
$$

For negative values of $c_{2}$ this equation represents two closed ovals which do not enclose the origin. If $c_{2} c^{2}=-1$ the ovals shrink upon the points $\rho=-1 / c_{2}$, $\phi=0$ and $\pi$. The corresponding orbit is therefore a circle in the equatorial plane. As $c_{2}$ approaches zero the ovals open out rapidly and approach the limiting curves

$$
\rho=\frac{c^{2}}{2 \cos ^{2} \bar{\phi}}
$$

For values of $c_{2}>0$ there is but one positive value for $\rho$, which is

$$
\rho=\frac{1}{c_{2}}\left[-1+\sqrt{1+\frac{c_{2} c^{2}}{\cos ^{2} \phi}}\right] \text {. }
$$

If $c^{2} \neq 0$ none of these curves crosses the axis $\phi=90^{\circ}$. But if $c=0$ we have the circle $\rho=-2 / c_{2}$ inside of which the motion is real when $c_{2}$ is negative. 
For values of $\mu^{2} \neq 0$, but sufficiently small, we can put

$$
r=(\rho+\bar{\rho}) \cos \phi, \quad q=(\rho+\bar{\rho}) \sin \phi,
$$

and solve for $\bar{\rho}$ as a power series in $\mu^{2}$. We find

$$
\bar{\rho}=\frac{1}{3} \frac{2-3 \cos ^{2} \phi}{\rho\left(1+c_{2} \rho\right)} \theta_{1}^{2} \mu^{2}+\cdots,
$$

which is the correction to be applied to the corresponding curves in the twobody problem.

\section{PART I.}

ORBITS WHICH REËNTER AFTER ONE REVOLUTION.

\section{§ 4. Symmetry.}

Returning to the differential equations $(6 a)$ and $(6 b)$ we observe that if we change

$$
r \text { into }+r, \quad q \text { into }-q, \quad \tau \text { into }-\tau,
$$

the differential equations remain unchanged. Hence, if at some epoch, $\tau=\tau_{0}$,

$$
\begin{array}{ll}
r=\alpha, & r^{\prime}=0, \\
q=0, & q^{\prime}=\beta,
\end{array}
$$

that is, if at the epoch $\tau=\tau_{0}$, the particle crosses the $r$-axis perpendicularly, it follows from the form of the differential equations that the orbit is symmetrical with respect to the $r$-axis and with respect to the epoch $\tau=\tau_{0}$. In other words $r$ will be an even series in $\left(\tau-\tau_{0}\right)$ and $q$ will be an odd series in $\left(\tau-\tau_{0}\right)$. If now at some other epoch, $\tau=\tau_{0}+T$, the particle again crosses the $r$-axis perpendicularly the orbit will be symnetrical with respect to this epoch also. It is clear therefore that the orbit is a closed one, and that the motion in it is periodic, for, by hypothesis, at $\tau=\tau_{0}-T$ it must have been at the same point and moving with the same velocity in the same direction. The motion is therefore periodic with the period $2 T$. Hence with these initial values sufficient conditions for periodicity are that at $\tau=\tau_{0}+T$

$$
r^{\prime}=q=0 \text {. }
$$

From the integral of area, $v^{\prime}=c / r^{2}$, it follows that if $r$ is periodic $v$ will have the form

$$
v=\text { constant } \times \tau+\text { periodic terms. }
$$


§5. Existence of periodic orbits in the equatorial plane.

If $q \equiv 0$ equations (6) reduce to

$$
\text { (a) } \quad r^{\prime \prime}=\frac{c^{2}}{r^{3}}-\frac{1}{r^{2}}-\frac{\theta_{1}^{2} \mu^{2}}{r^{4}}-\frac{\theta_{2}^{2} \mu^{4}}{r^{6}}-\cdots,
$$

(b) $v^{\prime}=\frac{c}{r^{2}}$.

The first of these equations is independent of the second and can be integrated separately. It represents motion in a straight line in the revolving plane. It admits the constant solution

$$
r_{0}=1, \quad c_{0}^{2}=1+\theta_{1}^{2} \mu^{2}+\theta_{2}^{2} \mu^{4}+\cdots,
$$

which represents a point in the revolving plane, or a circle in the equatorial plane. In order to investigate the oscillations about this point let us put

$$
r=1+e \rho, \quad c^{2}=c_{0}^{2}+e \epsilon,
$$

where $\rho$ is a variable whose initial value is arbitrarily assigned, $e$ is an arbitrary parameter corresponding to the eccentricity in the two-body problem, and $\epsilon$ is a parameter to be determined so that $\rho$ shall be periodic.

On substituting these values $(8 a)$ and expanding as power series in $e$, the terms independent of $e$ cancel out and it is possible to divide through by $e$. The equation then becomes

$$
\begin{aligned}
\rho^{\prime \prime}+\left[1-\theta_{1}^{2} \mu^{2}-3 \theta_{2}^{2} \mu^{4}+\cdots\right] \rho= & \epsilon\left[1-3 \rho e+6 \rho^{2} e^{2}-10 \rho^{3} e^{3}+\cdots\right] \\
& +\rho^{2} e\left[3-4 \theta_{1}^{2} \mu^{2}-15 \theta_{2}^{2} \mu^{4}+\cdots\right] \\
& +\rho^{3} e^{2}\left[-6+10 \theta_{1}^{2} \mu^{2}+46 \theta_{2}^{2} \mu^{4}+\cdots\right] \\
& +\rho^{4} e^{3}\left[10-20 \theta_{1}^{2} \mu^{2}-111 \theta_{2}^{2} \mu^{4}+\cdots\right]
\end{aligned}
$$

We can simplify this equation somewhat by dividing through by the coefficient of $\rho$ in the left member and then substituting

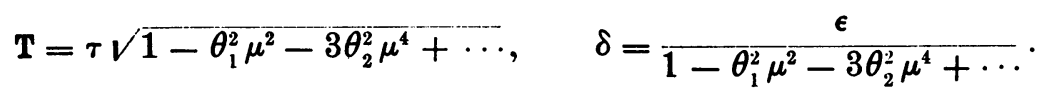

The equation then becomes

$$
\begin{aligned}
\frac{d^{2} \rho}{d \mathrm{~T}^{2}}+\rho=\delta\left[1-3 \rho e+6 \rho^{2} e^{2}-10 \rho^{3} e^{3}+\cdots\right]+\left[3+a_{1}\right] \rho^{2} e \\
+\left[-6+a_{2}\right] \rho^{3} e^{2}+\left[10+a_{3}\right] \rho^{4} e^{3}+\cdots
\end{aligned}
$$


where

$$
\begin{aligned}
& a_{1}=-3 \theta_{1}^{2} \mu^{2}-\left(\theta_{1}^{4}+6 \theta_{2}^{2}\right) \mu^{4}+\cdots, \\
& a_{2}=+4 \theta_{1}^{2} \mu^{2}+\left(4 \theta_{1}^{4}+28 \theta_{2}^{2}\right) \mu^{4}+\cdots, \\
& a_{3}=-10 \theta_{1}^{2} \mu^{2}-\left(10 \theta_{1}^{4}+81 \theta_{2}^{2}\right) \mu^{4}+\cdots
\end{aligned}
$$

Equation (10) can be integrated for $\rho$ as a power series in $\delta$ and $e$. Let us take the initial values

$$
\rho=-1, \quad \rho^{\prime}=0 .
$$

By Poincarés extension of Cauchy's theorem we know that the solution of equation (10) having the prescribed initial values exists and converges provided $\delta$ and $e$ are sufficiently small, for all values of $\mathrm{T}$ in the interval $0 \leqq \mathrm{~T} \leqq T$, where $T$ is finite, but otherwise arbitrary. The condition for periodicity is simply

$$
\rho^{\prime}=0 \text { at } \mathbf{T}=T .
$$

If we choose $T=\pi$ an inspection of equation (10) shows that for $e=0$ the solution for $\rho$ is periodic with the period $2 \pi$ whatever may be the value of $\delta$, so that equation (11) must carry $e$ as a factor. After integrating equation (10) we find that the condition (11) is

(12) $0=-\left[\frac{3}{2}+a_{1}\right] \pi \delta e-\left[\frac{3}{2}+\frac{5}{2} a_{1}+\frac{5}{12} a_{1}^{2}+\frac{3}{8} a_{2}\right] \pi e^{2}+$ higher degree terms.

Aside from the factor $e$ there remains an equation in which the linear terms in $e$ and $\delta$ are present, and which may be solved. We find

$$
\delta=(-1+\cdots) e+\cdots
$$

If this value of $\delta$ be substituted in equation (10) it will then admit periodic solutions for $\rho$ as power series in $e$ having the period $2 \pi$ for all values of $e$ sufficiently small. Furthermore the solution as a power series in $e$ with the prescribed initial conditions is unique.

\$6. Existence of periodic orbits not lying in the equatorial plane.

For $\mu^{2}=0$ the differential equations (6) admit the circular solution

$$
\begin{aligned}
& r=1, \quad q=0, \quad v=\tau, \\
& r^{\prime}=0, \quad q^{\prime}=0, \quad v^{\prime}=1, \\
& c^{2}=1 \text {. }
\end{aligned}
$$

In order to investigate the existence of orbits not lying in the equatorial plane but having the period $2 \pi$ for $\mu^{2} \neq 0$, let us put

$$
r=1+\rho, \quad q=0+\sigma, \quad c^{2}=1+\epsilon,
$$

and take the initial conditions

$$
\rho=\alpha, \quad \rho^{\prime}=0, \quad \sigma=0, \quad \sigma^{\prime}=\beta \mu .
$$


The conditions for periodicity $(\$ 4)$ are then

$$
\rho^{\prime}=\sigma=0 \text { at } \tau=\pi .
$$

We have three arbitrary constants at our disposal, $\alpha, \beta$, and $\epsilon$, and two conditions to be satisfied. Hence one constant remains undetermined. We will therefore let $\beta$ remain arbitrary and determine $\alpha$ and $\epsilon$ so as to satisfy the two conditions. After making the substitutions (15) and expanding, equations (6) become

$$
\begin{aligned}
& \rho^{\prime \prime}+\rho=\epsilon-3 \rho \epsilon+3 \rho^{2}+\frac{3}{2} \sigma^{2}-\theta_{1}^{2} \mu^{2}+6 \rho^{2} \epsilon-6 \rho^{3}-6 \rho \sigma^{2} \\
& +4 \rho \theta_{1}^{2} \mu^{2}+\text { higher degree terms, } \\
& \sigma^{\prime \prime}+\sigma=3 \rho \sigma-6 \rho^{2} \sigma+\frac{3}{2} \rho^{3}-\sigma \theta_{1}^{2} \mu^{2}+\text { higher degree terms. }
\end{aligned}
$$

In order to integrate these equations let us put

$$
\begin{aligned}
& \rho=\sum_{i, j, k=0}^{\infty} \rho_{i j k} \epsilon^{i} \alpha^{j} \mu^{k}, \\
& \sigma=\sum_{i, j, k=1}^{\infty} \sigma_{i j k} \epsilon^{i} \alpha^{j} \mu^{k} .
\end{aligned}
$$

The $\rho_{i j k}$ and $\sigma_{i j k}$ can be found by successive integrations, the constants of integration being determined so as to satisfy the initial conditions. In the series thus obtained take $\tau=\pi$. The two conditions for periodicity give us the two equations :

(a) $\rho_{\tau=\pi}^{\prime}=0=a_{1} \epsilon^{2}+a_{2} \epsilon \alpha+a_{3} \epsilon^{3}+a_{4} \epsilon^{2} \alpha+a_{5} \epsilon \alpha^{2}+a_{6} \alpha^{3}+a_{7} \epsilon \mu^{2}+a_{8} \alpha \mu^{2}+a_{9} \mu^{4}+\cdots$,

(b) $\sigma_{\tau=\pi}=0=\beta \mu\left[b_{1} \epsilon+b_{2} \epsilon^{2}+b_{3} \alpha^{2}+b_{4} \epsilon \alpha+b_{5} \mu^{2}+\cdots\right]$,

where the $a_{i}$ and $b_{i}$ are constants which have been found from the actual integrations to be distinct from zero. Equation (18a) involves only the even powers of $\mu$ while (18b) involves only the odd powers. After dividing (18b) by $\beta \mu$ we can solve it for $\epsilon$ as a power series in $\alpha$ and $\mu^{2}$ of the form

$$
\epsilon=c_{1} \alpha^{2}+c_{2} \mu^{2}+c_{3} \alpha^{3}+c_{4} \alpha \mu^{2}+c_{5} \mu^{4}+\cdots .
$$

On substituting (19) in (18a) we obtain a series of the form

$$
0=d_{1} \alpha \mu^{2}+d_{2} \alpha^{3}+d_{3} \mu^{4}+d_{4} \alpha^{2} \mu^{2}+d_{5} \alpha^{4}+\cdots
$$

If in this equation we make the substitution we obtain

$$
\alpha=\left(\gamma-\frac{d_{3}}{d_{1}}\right) \mu^{2},
$$

$$
0=\mu^{4}\left[f_{1} \gamma+f_{2} \mu^{2}+f_{3}^{\prime} \gamma \mu^{2}+\cdots\right],
$$


which can be solved uniquely for $\gamma$ as a power series in $\mu^{2}$. This solution substituted in (20b) gives $\alpha$ as a power series in $\mu^{2}$, and this value of $\alpha$ substituted in (19) gives $\epsilon$ as a power series in $\mu^{2}$. We thus have a solution

$$
\alpha=\mu^{2} P_{1}\left(\mu^{2}\right), \quad \epsilon=\mu^{2} P_{2}\left(\mu^{2}\right), \quad \beta \text { arbitrary. }
$$

Newton's parallelogram shows that equation $(20 \alpha)$ has two additional solutions, but as they are imaginary we do not stop to develop them.

\section{§7. Existence of periodic orbits in a meridian plane.}

If in equations (6) we take the area constant $c^{2}=0$ the motion of the particle is in a meridian plane; that is, the revolving plane has ceased to revolve and the orbit in this plane is the true orbit. After changing to polar coördinates by the substitution

$$
r=p \cos \phi, \quad q=p \sin \phi, \quad c^{2}=0,
$$

the differential equations become

$$
\begin{aligned}
p^{\prime \prime}-p \phi^{\prime 2}+\frac{1}{p^{2}} & =-\frac{-\frac{3}{4}+\frac{3}{2} \cos 2 \phi+\frac{1}{4} \cos 4 \phi}{p^{4}} \theta_{1}^{2} \mu^{2}+\cdots, \\
p \phi^{\prime \prime}+2 p^{\prime} \phi^{\prime} & =-\frac{\frac{1}{2} \sin 2 \phi-\frac{1}{4} \sin 4 \phi}{p^{4}} \theta_{1}^{2} \mu^{2}+\cdots
\end{aligned}
$$

For $\mu^{2}=0$ we have the periodic solution

$$
p=1, \quad \phi=\tau,
$$

that is, a circle. For $\mu^{2} \neq 0$ let us introduce $\rho$ and $\sigma$ by

$$
p=1+\rho, \quad \phi=\tau+\sigma,
$$

with the initial values

$$
\rho=\alpha, \quad \rho^{\prime}=0, \quad \sigma=0, \quad \sigma^{\prime}=\beta,
$$

where $\alpha$ and $\beta$ are two new arbitraries. By Poincaré's theorem $\rho, \rho^{\prime}, \sigma$ and $\sigma^{\prime}$ are expansible as power series in $\alpha, \beta$ and $\mu^{2}$ with $\tau$ entering the coefficients. The conditions for periodicity are that at $\tau=\pi$

$$
\rho^{\prime}=\sigma=0 \text {. }
$$

If we perform the integration and then set $\tau=\pi$, we obtain the two following conditional equations :

$$
\begin{aligned}
& \text { (a) } \sigma_{\tau=\pi}=0=a_{1} \alpha+a_{2} \beta+a_{3} \alpha^{2}+a_{4} \alpha \beta+a_{5} \beta^{2}+a_{6} \mu^{2}+\cdots, \\
& \text { (b) } \rho_{\tau=\pi}^{\prime}=0=
\end{aligned}
$$


where the $a_{i}$ and $b_{i}$ are constants which have been found from the actual integrations to be distinct from zero.

The first of these two equations can be solved for $\alpha$ as a power series in $\beta$ and $\mu^{2}$. This expression for $\alpha$ substituted in $(b)$ gives rise to an equation of the form

$$
0=c_{1} \beta \mu^{2}+c_{2} \beta^{3}+c_{3} \beta^{2} \mu^{2}+c_{4} \mu^{4}+\cdots
$$

This equation has the same form as $(20 a)$ and can be solved in the same way, giving a unique real value for $\beta$ as a power series in $\mu^{2}$ vanishing with $\mu^{2}$. This expression for $\beta$ substituted in the equation for $\alpha$ gives $\alpha$ as a power series in $\mu^{2}$, vanishing with $\mu^{2}$. Therefore real periodic orbits exist for $\mu^{2} \neq 0$ which are analytic continuations of circular orbits for $\mu^{2}=0$.

We have thus proved the existence of the three following classes of periodic orbits which have the period $2 \pi$, the generating orbits being circles :

I. Orbits lying in the equatorial plane ;

II. Orbits not lying in the equatorial plane;

III. Orbits in a meridian plane.

\section{§. Construction of periodic solutions in the equatorial plane.}

Let us first consider orbits in the equatorial plane. We retake the differential equations (8) and by means of the transformations there given we pass at once to equation (10), which is explicitly

$$
\begin{aligned}
\frac{d^{2} \rho}{d \mathrm{~T}^{2}}+\rho=\delta[1-3 \rho e & \left.+6 \rho^{2} e^{2}+\cdots\right]+\left[3-3 \theta_{1}^{2} \mu^{2}-\left(\theta_{1}^{4}+6 \theta_{2}^{2}\right) \mu^{4}+\cdots\right] \rho^{2} e \\
& +\left[-6+4 \theta_{1}^{2} \mu^{2}+\left(4 \theta_{1}^{2}+28 \theta_{2}^{2}\right) \mu^{4}+\cdots\right] \rho^{3} e^{2} \\
& +\left[10-10 \theta_{1}^{2} \mu^{2}-\left(10 \theta_{1}^{4}+81 \theta_{2}^{2}\right) \mu^{4}+\cdots\right] \rho^{4} e^{3}+\cdots
\end{aligned}
$$

It was shown in equation (13) that $\delta$ can be expanded uniquely as a power series in $e$ in such a manner that the solution for $\rho$ as a power series in $e$ will be periodic with the period $2 \pi$. Since the series is periodic with the same period for all values of $e$ sufficiently small, it follows that the coefficient of each power of $e$ is itself periodic. Since the solution exists and is unique, it must be possible to determine the $\delta$ uniquely by the condition that the solution is periodic. In the existence proof it was shown that $\delta$ vanishes with $e$. Therefore $\rho$ and $\delta$ have the form

$$
\rho=\rho_{0}+\rho_{1} e+\rho_{2} e^{2}+\rho_{3} e^{3}+\cdots, \quad \delta=\delta_{1} e+\delta_{2} e^{2}+\delta_{3} e^{3}+\cdots
$$

The $\rho_{j}$ are to be determined by the integration of equation (23) and by the initial values

$$
\rho=-1, \quad \frac{d \rho}{d \mathbf{T}}=0 \text { at } \mathbf{T}=0 .
$$

The $\delta_{j}$ are to be determined in such a manner that the $\rho_{j}$ shall be periodic. 
Substituting (24) in (23) and equating the coefficients, we find:

(a) $\frac{d^{2} \rho_{0}}{d \mathrm{~T}^{2}}+\rho_{0}=0$

(b) $\frac{d^{2} \rho_{1}}{d \mathbf{T}^{2}}+\rho_{1}=\delta_{1}+\left\lfloor 3-3 \theta_{1}^{2} \mu^{2}-\left(\theta_{1}^{4}+6 \theta_{2}^{2}\right) \mu^{4}+\cdots\right] \rho_{0}^{2}$,

(c) $\frac{d^{2} \rho_{2}}{d \mathbf{T}^{2}}+\rho_{2}=\delta_{2}-3 \rho_{0} \delta_{1}+\left[3-3 \theta_{1}^{2} \mu^{2}-\left(\theta_{1}^{4}+6 \theta_{2}^{2}\right) \mu^{4}+\cdots\right] 2 \rho_{0} \rho_{1}$ $+\left[-6+4 \theta_{1}^{2} \mu^{2}+\left(4 \theta_{1}^{4}+28 \theta_{2}^{2}\right) \mu^{4}+\cdots\right] \rho_{0}^{3}$,

(d) $\frac{d^{2} \rho_{n}}{d \mathrm{~T}^{2}}+\rho_{n}=\delta_{n}-3 \rho_{0} \delta_{n-1}+\left[3-3 \theta_{1}^{2} \mu^{2}-\left(\theta_{1}^{4}+6 \theta_{2}^{2}\right) \mu^{4}+\cdots\right] 2 \rho_{0} \rho_{n-1}$ $+f_{n}\left(\rho_{0}, \cdots, \rho_{n-2}\right)$,

These equations can be integrated in succession. The solution of $(a)$ which satisfies the initial conditions is

$$
\rho_{0}=-\cos \mathrm{T} \text {. }
$$

Since the initial conditions are independent of $e$, every $\rho_{j}$ except $\rho_{0}$ must vanish at $\mathbf{T}=0$. Substituting (26) in $(25 b)$ and integrating, we have

$$
\rho_{1}=\delta_{1}(1-\cos \mathrm{T})+\left[3-3 \theta_{1}^{2} \mu^{2}-\left(\theta_{1}^{4}+6 \theta_{2}^{2}\right) \mu^{4}+\cdots\right]\left[\frac{1}{2}-\frac{1}{3} \cos \mathrm{T}-\frac{1}{6} \cos 2 \mathrm{~T}\right] .
$$

The constants of integration in equation (27) have been determined so as to satisfy the initial conditions, but the constant $\delta_{1}$ is as yet undetermined.

Substituting these values of $\rho_{0}$ and $\rho_{1}$ in $(25 c)$, we find

$$
\begin{aligned}
\frac{d^{2} \rho_{2}}{d \mathrm{~T}^{2}}+\rho_{2}= & {\left[\delta_{2}+\delta_{1}\left(3-3 \theta_{1}^{2} \mu^{2}+\cdots\right)+3-6 \theta_{1}^{2} \mu^{2}+\cdots\right] } \\
& +\left[\delta_{1}\left(-3+6 \theta_{1}^{2} \mu^{2}+\left\{2 \theta_{1}^{4}+12 \theta_{2}^{2}\right\} \mu^{4}+\cdots\right)\right. \\
& \quad+\left(-3+12 \theta_{1}^{2} \mu^{2}-\left\{\frac{11}{2} \theta_{1}^{2}-9 \theta_{2}^{2}\right\} \mu^{4}+\cdots\right] \cos \mathrm{T} \\
& +\left[\delta_{1}\left(3-3 \theta_{1}^{2} \mu^{2}+\cdots\right)+3-18 \theta_{1}^{2} \mu^{2}+\cdots\right] \cos 2 \mathrm{~T} \\
& +\left[3-4 \theta_{1}^{2} \mu^{2}+\cdots\right] \cos 3 \mathrm{~T} .
\end{aligned}
$$

In order that the solution of this equation may be periodic the coefficient of $\cos \mathrm{T}$ must be zero. This is the condition which determines $\delta_{1}$ and consequently

$$
\delta_{1}=-1+2 \theta_{1}^{2} \mu^{2}+\left(\frac{3}{2} \theta_{1}^{+}-\theta_{2}^{2}\right) \mu^{4}+\cdots
$$


With this value of $\delta_{1}$ equation (28) becomes

$\frac{d^{2} \rho_{2}}{d \mathrm{~T}^{2}}+\rho_{2}=\left[\delta_{2}+3 \theta_{1}^{2} \mu^{2}+\cdots\right]+\left[-9 \theta_{1}^{2} \mu^{2}+\cdots\right] \cos 2 \mathrm{~T}+\left[3-4 \theta_{1}^{2} \mu^{2}+\cdots\right] \cos 3 \mathrm{~T}$.

The solution of this equation which satisfies the initial conditions is

$$
\begin{aligned}
\rho_{2}=\delta_{2}(1-\cos \mathbf{T})+\left[3 \theta_{1}^{2} \mu^{2}+\cdots\right]+\left[\frac{3}{8}-\frac{13}{2} \theta_{1}^{2} \mu^{2}+\cdots\right] \cos \mathbf{T} \\
+\left[3 \theta_{1}^{2} \mu^{2}+\cdots\right] \cos 2 \mathrm{~T}+\left[-\frac{3}{8}+\frac{1}{2} \theta_{1}^{2} \mu^{2}+\cdots\right] \cos 3 \mathrm{~T}
\end{aligned}
$$

The constant $\delta_{2}$ is as yet undetermined. It is determined by the periodicity condition for $\rho_{3}$ in the same manner that $\delta_{1}$ was determined by the periodicity condition of $\rho_{2}$. Without giving the details of the computation its value is found to be

$$
\delta_{2}=-6 \theta_{1}^{2} \mu^{2}+\cdots
$$

This method of integration can be carried as far as may be desired. In orderto show this let us suppose that $\rho_{0}, \cdots, \rho_{n-1}$ have been computed and all the constants determined with the exception of $\delta_{n-1}$. From (25d) we have

$$
\frac{d^{2} \rho_{n}}{d \mathbf{T}^{2}}+\rho_{n}=\delta_{n}-3 \rho_{0} \delta_{n-1}+\left[3-3 \theta_{1}^{2} \mu^{2}+\cdots\right] 2 \rho_{0} \rho_{n-1}+f_{n}\left(\rho_{0}, \cdots, \rho_{n-2}\right) .
$$

Here $f_{n}\left(\rho_{0}, \cdots, \rho_{n-2}\right)$ is a polynomial in the $\rho_{j}$ and contains only known terms. $\rho_{n-1}$ depends upon $\delta_{n-1}$ in the following way :

$$
\rho_{n-1}=\delta_{n-1}(1-\cos \mathrm{T})+\text { known terms. }
$$

Equation (30) can therefore be written

$$
\begin{array}{r}
\frac{d^{2} \rho_{n}}{d \mathbf{T}^{2}}+\rho_{n}=\delta_{n}+\left[3-3 \theta_{1}^{2} \mu^{2}+\cdots\right] \delta_{n-1}+\left[-3+6 \theta_{1}^{2} \mu^{2}+\cdots\right] \delta_{n-1} \cos \mathbf{T} \\
+\left[3-3 \theta_{1}^{2} \mu^{2}+\cdots\right] \cos 2 \mathrm{~T}+\text { known terms. }
\end{array}
$$

In order that the solution of this equation shall be periodic the coefficient of $\cos \mathrm{T}$ must be zero. This condition determines $\delta_{n-1}$. The equation can then be integrated and the constants of integration will be determined by the conditions that

$$
\rho_{n}=\frac{d \rho_{n}}{d \mathbf{T}}=0 \text { at } \mathbf{T}=0 .
$$

Everything is then determined with the exception of $\delta_{n}$, and we have then

$$
\rho_{n}=(1-\cos \mathrm{T}) \delta_{n}+\text { known terms. }
$$

Substituting the values of $\delta_{1}$ and $\delta_{2}$ in the solution as far as it has been com- 
puted, we find

$$
\begin{aligned}
& \rho_{0}=-\cos \mathbf{T}, \\
& \begin{aligned}
\rho_{1}= & {\left[\frac{1}{2}+\frac{1}{2} \theta_{1}^{2} \mu^{2}+\left(\theta_{1}^{4}-4 \theta_{2}^{2}\right) \mu^{4} \cdots\right]+\left[-\theta_{1}^{2} \mu^{2}+\left(-\frac{7}{6} \theta_{1}^{4}+3 \theta_{2}^{2}\right) \mu^{4}+\cdots\right] \cos \mathbf{T} } \\
\quad & \quad\left[-\frac{1}{2}+\frac{1}{2} \theta_{1}^{2} \mu^{2}+\left(\frac{1}{6} \theta_{1}^{4}+\theta_{2}^{2}\right) \mu^{4} \cdots\right] \cos 2 \mathrm{~T},
\end{aligned} \\
& \begin{array}{c}
\rho_{2}=\left[-3 \theta_{1}^{2} \mu^{2}+\cdots\right]+\left[\frac{3}{8}-\frac{1}{2} \theta_{1}^{2} \mu^{2}+\cdots\right] \cos \mathrm{T}+\left[3 \theta_{1}^{2} \mu^{2}+\cdots\right] \cos 2 \mathrm{~T} \\
\quad+\left[-\frac{3}{8}+\frac{1}{2} \theta_{1}^{2} \mu^{2}+\cdots\right] \cos 3 \mathrm{~T},
\end{array} \\
& \delta_{1}=-1+2 \theta_{1}^{2} \mu^{2}+\left(\frac{3}{2} \theta_{1}^{4}-\theta_{2}^{2}\right) \mu^{4}+\cdots, \\
& \delta_{2}=-6 \theta_{1}^{2} \mu^{2}+\cdots .
\end{aligned}
$$

From these expressions we have the following series for $r=1+e \rho$ :

(a) $r=1-e \cos \mathrm{T}+\left\{\left[\frac{1}{2}+\frac{1}{2} \theta_{1}^{2} \mu^{2}+\left(\theta_{1}^{4}-4 \theta_{2}^{2}\right) \mu^{4}+\cdots\right]\right.$

$$
\begin{aligned}
& +\left[-\theta_{1}^{2} \mu^{2}+\left(-{ }_{6}^{7} \theta_{1}^{4}+3 \theta_{2}^{2}\right) \mu^{4}+\cdots\right] \cos \mathrm{T} \\
& \left.+\left[-\frac{1}{2}+\frac{1}{2} \theta_{1}^{2} \mu^{2}+\left(\frac{1}{6} \theta_{1}^{4}+\theta_{2}^{2}\right) \mu^{4}+\cdots\right] \cos 2 \mathrm{~T}\right\} e^{2} \\
& +\left\{\left[-3 \theta_{1}^{2} \mu^{2}+\cdots\right]+\left[\frac{3}{8}-\frac{1}{2} \theta_{1}^{2} \mu^{2}+\cdots\right] \cos \mathrm{T}\right. \\
& \left.+\left[3 \theta_{1}^{2} \mu^{2}+\cdots\right] \cos 2 \mathrm{~T}+\left[-\frac{3}{8}+\frac{1}{2} \theta_{1}^{2} \mu^{2}+\cdots\right] \cos 3 \mathrm{~T}\right\} e^{3}+\cdots
\end{aligned}
$$

Substituting this value of $r$ in the equation (8b)

$$
r^{2} \frac{d v}{d \mathrm{~T}}=\sqrt{\frac{c_{0}^{2}+e \epsilon}{1-\theta_{1}^{2} \mu^{2}-3 \theta_{2}^{2} \mu^{4} \ldots}},
$$

and integrating, we find

$$
\begin{aligned}
(b) v-v_{0}= & \left\{\left[1+\theta_{1}^{2} \mu^{2}+\left(\frac{1}{2} \theta_{1}^{4}+2 \theta_{2}^{2}\right) \mu^{4}+\cdots\right]\right. \\
& \left.+\left[\theta_{1}^{2} \mu^{2}+\left(-\frac{9}{4} \theta_{1}^{4}+\frac{19}{2} \theta_{2}^{2}\right) \mu^{4}+\cdots\right] e^{2}+\cdots\right\} \mathbf{T} \\
& +\left\{\left[2+2 \theta_{1}^{2} \mu^{2}+\left(\theta_{1}^{4}+\theta_{2}^{2}\right) \mu^{4}+\cdots\right] \sin \mathbf{T}\right\} e \\
& +\left\{\left[2 \theta_{1}^{2} \mu^{2}+\left(\frac{13}{3} \theta_{1}^{4}-6 \theta_{2}^{2}\right) \mu^{4}+\cdots\right] \sin \mathbf{T}\right. \\
& \left.+\left[\frac{5}{4}+\frac{3}{4} \theta_{1}^{2} \mu^{2}+\left(-\frac{1}{2} \theta_{1}^{4}+\frac{3}{2} \theta_{2}^{2}\right) \mu^{4}+\cdots\right] \sin 2 \mathbf{T}\right\} e^{2}+\cdots
\end{aligned}
$$

Equations (31a) and (31b) are the periodic solutions sought. If we return to the symbols defined in the original differential equations by means of equations (4), with the additional notation

$$
n V \overline{1-\theta_{1}^{2} \mu^{2}-3 \theta_{2}^{2}} \overline{\mu^{4}}+\cdots=\nu,
$$


we have the following expressions for the polar coördinates at any time $t$ :

$$
\begin{aligned}
R= & a\left\{1-e \cos \nu t+\left[\frac{1}{2}-\frac{1}{2} \cos 2 \nu t\right] e^{2}+\left[\frac{3}{8} \cos \nu t-\frac{3}{8} \cos 3 \nu t\right] e^{3}+\cdots\right. \\
& +\frac{b^{2}}{a^{2}} \mu^{2}\left[\left(\frac{3}{20}-\frac{3}{10} \cos \nu t+\frac{3}{20} \cos 2 \nu t\right) e^{2}+\left(-\frac{9}{10}-\frac{3}{20} \cos \nu t\right.\right. \\
& \left.\left.\left.+\frac{9}{10} \cos 2 \nu t+\frac{3}{20} \cos 3 \nu t\right) e^{3}+\cdots\right]+\frac{b^{4}}{a^{4}} \mu^{4}[\cdots]+\cdots\right\} \\
v-v_{0}= & \nu t+2 \sin \nu t \cdot e+\frac{5}{4} \sin 2 \nu t \cdot e^{2}+\cdots+\frac{b^{2}}{a^{2}} \mu^{2}\left[\left(\frac{3}{10}+\frac{3}{10} e^{2}+\cdots\right) \nu t\right. \\
& \left.+\left(\frac{3}{5} \sin \nu t\right) e^{2}+\left(\frac{3}{5} \sin \nu t+\frac{9}{40} \sin 2 \nu t\right) e^{2}+\cdots\right]+\frac{b^{4}}{a^{4}} \mu^{4}[\cdots]+\cdots
\end{aligned}
$$

These equations contain four arbitrary constants, $a, e, v_{0}$ and $t_{0}$. Since the differential equations of motion in the equatorial plane were of the fourth order these series, within the realm of their convergence, represent the general solution. The expression for the radius vector, $R$, is always periodic with the period $2 \pi / \nu$. At the expiration of this period $v$ has increased by the quantity

$$
2 \pi\left[\frac{b^{2}}{a^{2}} \mu^{2}\left(\frac{-3}{1} \sigma+\frac{3}{10} e^{2}+\cdots\right)+\cdots\right]=2 \pi \Theta
$$

in excess of $2 \pi$; that is, the line of apsides has rotated forwarded by this amount. If $\Theta$ is commensurable with unity the orbit is eventually closed geometrically. If $\Theta=I / J$, where $I$ and $J$ are integers relatively prime, then, at $t=2 J \pi / \nu, v=2(I+J) \pi$, and the particle is at its initial position with its initial components of velocity. The particle has completed $I+J$ revolutions and the line of apsides has completed $I$ revolutions. The mean sidereal period is

$$
P=\frac{2 \pi}{\nu(1+\Theta)} .
$$

This formula for the rotation of the line of apsides has an interesting application in the case of Jupiter's fifth satellite. On the hypothesis that Jupiter is homogeneous and taking its equatorial diameter as 90,190 miles and its polar diameter as 84,570 miles, the mean distance of the satellite as 112,500 miles, the eccentricity of its orbit as .006 and its mean sidereal period as $11^{\mathrm{h}} 0^{\mathrm{m}} 23^{\mathrm{s}}$, the above formula gives for the rotation of the line of apsides $1440^{\circ}$ per year. The values derived from observations are somewhat discordant but are in the neighborhood of $880^{\circ}$ per year. If we still keep the hypothesis that Jupiter is homogeneous in density and of the same oblateness as before, we are compelled, in order to relieve the discrepancy between theory and observation, to suppose that the value adopted for its polar radius was about 9,000 miles too 
great. From the large reduction required it is clear that the hypothesis of homogeneity is very much in error.

\section{§9. Construction of periodic solutions not lying in the equatorial plane.}

By means of the area integral the problem has been reduced to the two equations

$$
\begin{aligned}
& r^{\prime \prime}=\frac{c^{2}}{r^{3}}-\frac{r}{\left(r^{2}+q^{2}\right)^{\frac{3}{2}}}-\frac{r^{3}-4 r q^{2}}{\left(r^{2}+q^{2}\right)^{\frac{7}{2}}} \theta_{1}^{2} \mu^{2}+\cdots, \\
& q^{\prime \prime}=-\frac{q}{\left(r^{2}+q^{2}\right)^{\frac{3}{2}}}-\frac{3 r^{2} q-2 q^{3}}{\left(r^{2}+q^{2}\right)^{\frac{7}{2}}} \theta_{1}^{2} \mu^{2}+\cdots
\end{aligned}
$$

After the solution of these equations has been obtained the third coördinate is found from the equation

$$
v^{\prime}=\frac{c}{r^{2}}
$$

We have already proved [equations (14) to (20)] the existence of periodic solutions of these equations of the following type:

$$
\begin{aligned}
r & =1+\rho_{2} \mu^{2}+\rho_{4} \mu^{4}+\cdots, \\
q & =q_{1} \mu+q_{3} \mu^{3}+q_{5} \mu^{5}+\cdots, \\
c^{2} & =1+c_{2} \mu^{2}+c_{4} \mu^{4}+\cdots,
\end{aligned}
$$

with the initial conditions

$$
r^{\prime}(0)=q(0)=0, \quad q^{\prime}(0)=\beta \mu,
$$

$\beta$ being arbitrary. We can therefore integrate equations (35) so as to satisfy these initial conditions and determine the $c_{j}$ in such a manner as to render the solution periodic.

Substituting (36) in (35) gives the equations

$$
\begin{array}{r}
\begin{array}{r}
0=\left[\rho_{2}^{\prime \prime}+\rho_{2}-\frac{3}{2} q_{1}^{2}-c_{2}+\theta_{1}^{2}\right] \mu^{2}+\left[\rho_{4}^{\prime \prime}+\rho_{4}-3 \rho_{2}^{2}-3 q_{1} q_{3}+6 \rho_{2} q_{1}^{2}\right. \\
\left.+\frac{15}{8} q_{1}^{4}+\left(3 c_{2}-4 \theta_{1}^{2}\right) \rho_{2}-\frac{15}{2} \theta_{1}^{2} q_{1}^{2}-c_{4}\right] \mu^{4}+\cdots,
\end{array} \\
\begin{array}{r}
0=\left[q_{1}^{\prime \prime}+q_{1}\right] \mu+\left[q_{3}^{\prime \prime}+q_{3}-3 \rho_{2} q_{1}-\frac{3}{2} q_{1}^{3}+3 \theta_{1}^{2} q_{1}\right] \mu^{3}+\left[q_{5}^{\prime \prime}+q_{5}-3 \rho_{2} q_{3}-\frac{9}{2} q_{1}^{2} q_{3}\right. \\
\left.+6 \rho_{2}^{2} q_{1}-3 \rho_{4} q_{1}+\frac{15}{2} \rho_{2} q_{1}^{3}+\frac{15}{8} q_{1}^{5}+3 \theta_{1}^{2} q_{3}-15 \theta_{1}^{2} \rho_{2} q_{1}-\frac{25}{2} \theta_{1}^{2} q_{1}^{3}\right] \mu^{5}+\cdots
\end{array}
\end{array}
$$

Equation (37) contains only even powers of $\mu$ while equation (38) contains only odd powers. For the integration we have :

Coefficient of $\mu$.

$$
q_{1}^{\prime \prime}+q_{1}=0
$$


The solution of this equation satisfying the initial conditions is

Coefficient of $\mu^{2}$.

$$
q_{1}=\beta \sin \tau \text {. }
$$

$$
\begin{aligned}
\rho_{2}^{\prime \prime}+\rho_{2} & =\frac{3}{2} q_{1}^{2}+c_{2}-\theta_{1}^{2}, \\
& =\left(\frac{3}{4} \beta^{2}+c_{2}-\theta_{1}^{2}\right)-\frac{3}{4} \beta^{2} \cos 2 \tau .
\end{aligned}
$$

The solution of this equation which satisfies the assigned initial conditions is

$$
\rho_{2}=\left(\frac{3}{4} \beta^{2}+c_{2}-\theta_{1}^{2}\right)+\alpha_{2} \cos \tau+\frac{1}{4} \beta^{2} \cos 2 \tau .
$$

The constant $c_{2}$ will be determined by the periodicity condition on $q_{3}$ to be

$$
c_{2}=2 \theta_{1}^{2}-\beta^{2},
$$

and $\alpha_{2}$ will be determined by the periodicity condition on $\rho_{4}$ to be

$$
\alpha_{2}=0 \text {. }
$$

If we anticipate these determinations we have

$$
\rho_{2}=\left(\theta_{1}^{2}-\frac{1}{4} \beta^{2}\right)+\frac{1}{4} \beta^{2} \cos 2 \tau \text {. }
$$

Coefficient of $\mu^{3}$.

$$
\begin{aligned}
q_{3}^{\prime \prime}+q_{3} & =q_{1}\left(3 \rho_{2}+\frac{3}{2} q_{1}^{2}-3 \theta_{1}^{2}\right), \\
& =\left(3 \beta^{2}+3 c_{2}-6 \theta_{1}^{2}\right) \beta \sin \tau+\frac{3}{4} \alpha_{2} \beta \sin 2 \tau .
\end{aligned}
$$

In order that the solution shall be periodic it is necessary that the coefficient of $\sin \tau$ shall be zero. Therefore

$$
c_{2}=2 \theta_{1}^{2}-\beta^{2} .
$$

Substituting this value and integrating, we find

$$
q_{3}=\beta_{3} \sin \tau-\frac{1}{4} \alpha_{2} \beta \sin 2 \tau \text {. }
$$

From the initial conditions we must have $q_{3}^{\prime}(0)=0$ and therefore

$$
\beta_{3}=\frac{1}{2} \alpha_{2} \beta \text {. }
$$

But it will be shown in the next step that $\alpha_{2}=0$, and consequently

Coefficient of $\mu^{4}$.

$$
q_{3} \equiv 0 \text {. }
$$

$$
\rho_{4}^{\prime \prime}+\rho_{4}=3 \rho_{2}^{2}+3 q_{1} q_{3}-6 \rho_{2} q_{1}^{2}-\frac{15}{8} q^{4}+\left(4 \theta_{1}^{2}-3 c_{2}\right) \rho_{2}+\frac{15}{2} \theta_{1}^{2} q_{1}^{2}+c_{4} .
$$

Before expanding the right member of this equation we will avoid useless labor by first examining the coefficient of $\cos \tau$ which we know must be zero from the periodicity condition. We notice first that terms in the coefficient of $\cos \tau$ can 
arise only through terms involving $\rho_{2}$ and $q_{3}$, and second that all such terms carry $\alpha_{2}$ as a factor. Since no other arbitrary enters the coefficient, we must take $\alpha_{2}=0$. It can be shown by induction that the constant of integration $\alpha_{2 i}$ (the coefficient of $\cos \tau$ ) which arises in the integration of $\rho_{2 i}$ is determined by the periodicity condition on $\rho_{2(i+1)}$, and further that its value is always zero. The proof is omitted for the sake of brevity.

Substituting the value $\alpha_{2}=0$ in $\rho_{2}$ and $q_{3}$ and expanding the right member of (42), we find

$\rho_{4}^{\prime \prime}+\rho_{4}=\left[c_{4}+\theta_{1}^{2}+\frac{11}{4} \theta_{1}^{2} \beta^{2}-\frac{3}{6} \frac{3}{4} \beta^{4}\right]+\left[\frac{1}{4} \theta_{1}^{2} \beta^{2}-\frac{3}{16} \beta^{4}\right] \cos 2 \tau+\frac{15}{6} \frac{5}{4} \beta^{4} \cos 4 \tau$.

The solution of this equation satisfying the initial conditions and the properties just mentioned is

$\rho_{4}=\left[c_{4}+\theta_{1}^{2}+\frac{11}{4} \theta_{1}^{2} \beta^{2}-\frac{3}{64} \beta^{4}\right]+\left[-\frac{1}{12} \theta_{1}^{2} \beta^{2}+\frac{1}{16} \beta^{4}\right] \cos 2 \tau-\frac{1}{64} \beta^{4} \cos 4 \tau$.

Anticipating the value of $c_{4}$ found below, we have

$\rho_{4}=\left[-3 \theta_{1}^{4}-\frac{11}{12} \theta_{1}^{2} \beta^{2}-\frac{3}{64} \beta^{4}\right]+\left[-\frac{1}{12} \theta_{1}^{2} \beta^{2}+\frac{1}{16} \beta^{2}\right] \cos 2 \tau-\frac{1}{64} \beta^{4} \cos 4 \tau$.

Coefficient of $\mu^{5}$.

$$
q_{5}^{\prime \prime}+q_{5}=\grave{s} \rho_{2} q_{3}+\frac{9}{2} q_{1}^{2} q_{3}-6 \rho_{2}^{2} q_{1}+3 \rho_{4} q_{1}-\frac{15}{2} \rho_{2} q_{1}^{2}-\frac{15}{8} q_{1}^{5}
$$

$$
\begin{aligned}
& -3 \theta_{1}^{2} q_{3}+15 \theta_{1}^{2} \rho_{2} q_{1}+\frac{25}{2} \theta_{1}^{2} q_{1}^{3}, \\
& =\left[3 c_{1}+12 \theta_{1}^{4}+11 \theta_{1}^{2} \beta^{2}\right] \beta \sin \tau-\theta_{1}^{2} \beta^{3} \sin 3 \tau .
\end{aligned}
$$

From the periodicity condition we have

$$
c_{4}=-4 \theta_{1}^{4}-\frac{11}{3} \theta_{1}^{2} \beta^{2} .
$$

Integrating and imposing the initial conditions, we find

$$
q_{5}=-\frac{3}{8} \theta_{1}^{2} \beta^{3} \sin \tau+\frac{1}{8} \theta_{1}^{2} \beta^{3} \sin 3 \tau .
$$

This is sufficient to make evident the general character of the series. The $r$ equation contains only even multiples of $\tau$, and the $q$-equation contains only odd multiples. The $r$-equation contains only even powers of $\mu$ and of $\tau$. The $q$ equation is odd in both these respects. The series are therefore triply even and odd.

Collecting the various coefficients we have the following series:

$$
\begin{array}{r}
r=1+\left[\left(\theta_{1}^{2}-\frac{1}{4} \beta^{2}\right)+\frac{1}{4} \beta^{2} \cos 2 \tau\right] \mu^{2}+\left[\left(-3 \theta_{1}^{4}-\frac{1}{1} \frac{1}{2} \theta_{1}^{2} \beta^{2}-\frac{3}{64} \beta^{4}\right)\right. \\
\left.+\left(-\frac{1}{12} \theta_{1}^{2} \beta^{2}+\frac{1}{16} \beta^{4}\right) \cos 2 \tau-\frac{1}{6} \frac{1}{4} \beta^{4} \cos 4 \tau\right] \mu^{4}+\cdots,
\end{array}
$$

(b) $q=[\beta \sin \tau] \mu+[0] \mu^{3}+\left[-\frac{3}{8} \theta_{1}^{2} \beta^{3} \sin \tau+\frac{1}{8} \theta_{1}^{2} \beta^{3} \sin 3 \tau\right] \mu^{5}+\cdots$,

(c) $v-v_{0}=\left[1-\theta_{1}^{2} \mu^{2}-\left(\frac{3}{2} \theta_{1}^{4}+\frac{1}{6} \theta_{1}^{2} \beta^{2}\right) \mu^{4}+\cdots\right] \tau+\left[-\frac{1}{4} \beta^{2} \sin 2 \tau\right] \mu^{2}$

$$
+\left[\left(\frac{1}{1} \frac{1}{2} \theta_{1}^{2} \beta^{2}-\frac{1}{8} \beta^{4}\right) \sin 2 \tau+\frac{1}{64} \beta^{4} \sin 4 \tau\right] \mu^{4}+\cdots,
$$

(d) $\quad c^{2}=1+\left[2 \theta_{1}^{2}-\beta^{2}\right] \mu^{2}+\left[-4 \theta_{1}^{4}-\frac{11}{3} \theta_{1}^{2} \beta^{2}\right] \mu^{4}+\cdots$ 
This solution contains four arbitrary constants, $a, \beta, v_{0}$ and $\tau_{0}$. As is shown by equation (44c) the nodes regress, the rate of regression being

$$
\left[\theta_{1}^{2} \mu^{2}+\left({ }_{2}^{3} \theta_{1}^{4}+\frac{1}{6} \theta_{1}^{2} \beta^{2}\right) \mu^{4}+\cdots\right] .
$$

The generating orbit of these solutions is a circle in the equatorial plane. A circle having any assigned inclination might have been used, e. g.,

$$
r=\sqrt{1-s^{2} \sin ^{2} \tau}, \quad q=s \sin \tau, \quad v=\tan ^{-1}\left[\gamma^{\prime} 1-s^{2} \tan \tau\right],
$$

where $s$ is the sine of the inclination. The solution thus obtained would have been identical with (44). If we expand (45) as power series in $s$ and then put $s=\beta \mu$, it will be found that the terms thus obtained are identical with the terms independent of $\theta_{1}^{2}$ in the solution which has been worked out. It might therefore be of assistance in the physical interpretation of the constants to put $\beta \mu=s$ in the series (44).

\section{\$10. Construction of periodic solutions in a meridian plane.}

When the constant $c$ is zero the motion is in a meridian plane. The equations of motion are (21)

$$
\begin{aligned}
p^{\prime \prime}-p \phi^{\prime 2}+\frac{1}{p^{2}} & =-\frac{-\frac{3}{4}+\frac{3}{2} \cos 2 \phi+\frac{1}{4} \cos 4 \phi}{p^{4}} \theta_{1}^{2} \mu^{2}+\cdots, \\
p \phi^{\prime \prime}+2 p^{\prime} \phi^{\prime} & =-\frac{\frac{1}{2} \sin 2 \phi-\frac{1}{4} \sin 4 \phi}{p^{4}} \theta_{1}^{2} \mu^{2}+\cdots
\end{aligned}
$$

We have already shown the existence of periodic solutions of these equations as power series in $\mu^{2}$, which for $\mu=0$ reduce to the circle $p=1, \phi=\tau$. Let us put then

$$
p=1+p_{2} \mu^{2}+p_{4} \mu^{4}+\cdots, \quad \phi=\tau+\phi_{2} \mu^{2}+\phi_{4} \mu^{4}+\cdots
$$

Substituting these expressions in (46), expanding and collecting the coefficients of the various powers of $\mu$, we find:

$$
\begin{gathered}
0=\left[p_{2}^{\prime \prime}-3 p_{2}-2 \phi_{2}^{\prime}-\frac{3}{4} \theta_{1}^{2}+\frac{3}{2} \theta_{1}^{2} \cos 2 \tau+\frac{1}{4} \theta_{1}^{2} \cos 4 \tau\right] \mu^{2}+\left[p_{4}^{\prime \prime}-3 p_{4}-2 \phi_{4}^{\prime}-2 p_{2} \phi_{2}^{\prime}\right. \\
\left.-\phi_{2}^{\prime 2}+3 p_{2}^{2}+(3-6 \cos 2 \tau-\cos 4 \tau) \theta_{1}^{2} p_{2}+(-3 \sin 2 \tau-\sin 4 \tau) \theta_{1}^{2} \phi_{2}\right] \mu^{4}+\cdots \\
0=\left[\phi_{2}^{\prime \prime}+2 p_{2}^{\prime}+\frac{1}{2} \theta_{1}^{2} \sin 2 \tau-\frac{1}{4} \theta_{1}^{2} \sin 4 \tau\right] \mu^{2}+\left[\phi_{4}^{\prime \prime}+2 p_{4}^{\prime}+\phi_{2}^{\prime \prime} p_{2}+2 p_{2}^{\prime} \phi_{2}^{\prime}\right. \\
\left.+(-2 \sin 2 \tau+\sin 4 \tau) \theta_{1}^{2} p_{2}+(\cos 2 \tau-\cos 4 \tau) \theta_{1}^{2} \phi_{2}\right] \mu^{4}+\cdots
\end{gathered}
$$

The initial conditions are

$$
p^{\prime}(0)=\phi(0)=0
$$


Proceeding to the integration we have :

Coefficient of $\mu^{2}$.

(a) $p_{2}^{\prime \prime}-3 p_{2}-2 \phi_{2}^{\prime}=\frac{3}{4} \theta_{1}^{2}-\frac{3}{2} \theta_{1}^{2} \cos 2 \tau-\frac{1}{4} \theta_{1}^{2} \cos 4 \tau$,

(b) $\quad \phi_{2}^{\prime \prime}+2 p_{2}^{\prime}=-\frac{1}{2} \theta_{1}^{2} \sin 2 \tau+\frac{1}{4} \theta_{1}^{2} \sin 4 \tau$.

By integrating $(b)$ once we have

$$
\phi_{2}^{\prime}=-2 p_{2}+\frac{1}{4} \theta_{1}^{2} \cos 2 \tau-\frac{1}{16} \theta_{1}^{2} \cos 4 \tau+c_{1} .
$$

Substituting this value of $\phi_{2}^{\prime}$ in $(a)$, we find

$$
p_{2}^{\prime \prime}+p_{2}=\left(2 c_{1}+\frac{3}{4} \theta_{1}^{2}\right)-\theta_{1}^{2} \cos 2 \tau-\frac{3}{8} \theta_{1}^{2} \cos 4 \tau .
$$

The integration of this equation gives

$$
p_{2}=\left(2 c_{1}+\frac{3}{4} \theta_{1}^{2}\right)+c_{2} \sin \tau+c_{3} \cos \tau+\frac{1}{3} \theta_{1}^{2} \cos 2 \tau+\frac{1}{40} \theta_{1}^{2} \cos 4 \tau .
$$

Since $p_{2}^{\prime}=0$ at $\tau=0$ we must take $c_{2}=0$. Then substituting this value of $p_{2}$ in $(c)$ and integrating, we get

$$
\text { (f) } \phi_{2}=\left(-3 c_{1}-\frac{3}{2} \theta_{1}^{2}\right) \tau-2 c_{3} \sin \tau-\frac{5}{24} \theta_{1}^{2} \sin 2 \tau-\frac{9}{32} \theta_{1}^{2} \sin 4 \tau+c_{4} \text {. }
$$

From the initial conditions $\phi_{2}$ must be zero when $\tau=0$. Therefore $c_{4}=0$. Since it must also be periodic, $c_{1}=-\frac{1}{2} \theta_{1}^{2}$. All of the constants of integration are now determined except $c_{3}$ which will be determined by the periodicity condition on $p_{4}$.

The differential equations for $p_{4}$ and $\phi_{4}$ are the same as for $p_{2}$ and $\phi_{2}$ except in the right members. The process of integration is therefore the same. In the right members only even multiples of $\tau$ occur except terms carrying the undetermined $c_{3}$ as a factor. In the equation corresponding to $(48 d)$ there will be a term in $\cos \tau$ carrying $c_{3}$ as a factor. But the integration of this term will be non-periodic unless $c_{3}=0$. Put then $c_{3}=0$; the integration proceeds just as before and the constants are determined in the same manner. This argument will be repeated in the coefficients of $\mu^{6}$ and so on for all higher powers.* Therefore no odd multiples of $\tau$ can occur in the solution. We have, therefore,

$$
\begin{aligned}
& p=1+\left[-\frac{1}{4}+\frac{1}{3} \cos 2 \tau+\frac{1}{40} \cos 4 \tau\right] \theta_{1}^{2} \mu^{2}+\cdots, \\
& q=\tau+\left[-\frac{5}{24} \sin 2 \tau-\frac{9}{320} \sin 4 \tau\right] \theta_{1}^{2} \mu^{2}+\cdots .
\end{aligned}
$$

\footnotetext{
* The differential equations arising at the successive steps are of the same type as the first two equations of (32), p. 555, in Professor Moulton's paper on periodic solutions of the problem of three bodies, these Transactions, vol. 7 (1906). Consequently his general formulas for the coefficients of the solutions, equations (42), loc. cit., apply here.
} 
Since the series involve only even multiples of $\tau$ the orbits are symmetrical with respect to both the $r$-axis and the $q$-axis.

This completes the formal construction of the solutions of which the existence was proved in $\S \S 5,6$ and 7 .

\section{Part II.}

\section{ORBITS REËNTRANT ONLY AFTER MANY REVOLUTIONS.}

\section{\$11. The differential equations.}

The orbits which we have previously considered have had the common property of involving only the period $2 \pi$. Since this period is independent of the oblateness of the spheroid the derivation of these orbits has been relatively simple. We shall proceed now to investigate a class of orbits which involves beside the period $2 \pi$ another period $2 \pi / \lambda$, where $\lambda$ is a function of the oblateness of the spheroid, the inclination of the orbit and the mean distance of the particle. We shall start from the solution which involves an arbitrary inclination. Into this solution four arbitrary constants were introduced, viz., inclination, mean distance, longitude of node and the epoch. Two more arbitraries are necessary for a complete solution, viz., constants corresponding to the eccentricity and to the longitude of perihelion from the node. In what follows we shall introduce the constant corresponding to the eccentricity, but we shall still project the particle from an apse at the node.

We have found for the differential equations a certain solution (44) which we may write

$$
r=\phi(\beta, \mu ; \tau), \quad q=\psi(\beta, \mu ; \tau), \quad c^{2}=c_{0}^{2},
$$

which is symmetric with respect to the equatorial plane. That is to say, at $\tau=0$ the particle is in the equatorial plane and its motion is perpendicular to the radius vector. Its initial distance is $\phi(0)$. Suppose now we change the initial distance slightly and also the initial velocity so that at $\tau=0$

$$
\begin{aligned}
& r(0)=\phi(0)+\alpha, \quad q(0)=0, \\
& r^{\prime}(0)=0, \quad q^{\prime}(0)=\psi^{\prime}(0)+\gamma,
\end{aligned}
$$

and give an increment to the constant of areas so that $c^{2}=c_{0}^{2}+\epsilon$. Can we determine these three constants, $\alpha, \gamma$ and $\epsilon$, in such a manner that the series for $r$ and $q$ shall be periodic? These series can be expressed by

$$
r=\phi(\beta, \mu ; \tau)+\rho, \quad q=\psi(\beta, \mu ; \tau)+\sigma, \quad c^{2}=c_{0}^{2}+\epsilon .
$$

If now we substitute these expressions in the differential equations (6) all the terms independent of $\rho, \sigma$ and $\epsilon$ will drop out, and there will remain the fol- 
lowing differential equations for $\rho$ and $\sigma$ :

(a) $\rho^{\prime \prime}+\left\{1+\left[\left(-4 \theta_{1}^{2}+\frac{3}{2} \beta^{2}\right)-{ }_{2}^{9} \beta^{2} \cos 2 \tau\right] \mu^{2}+\left[\left(20 \theta_{1}^{4}+6 \theta_{1}^{2} \beta^{2}+\frac{3}{8} \beta^{4}\right)\right.\right.$

$\left.\left.+\left(\theta_{1}^{2} \beta^{2}-\frac{3}{2} \beta^{4}\right) \cos 2 \tau+\frac{9}{8} \beta^{4} \cos 4 \tau\right] \mu^{4}+\cdots\right\} \rho+\{-3 \beta \mu \sin \tau$

$\left.+\left[\left(-3 \theta_{1}^{2} \beta+\frac{9}{8} \beta^{3}\right) \sin \tau-\frac{3}{8} \beta^{3} \sin 3 \tau\right] \mu^{3}+\cdots\right\} \sigma$

$+\epsilon\left\{\left\{1+\left[\left(-3 \theta_{1}^{2}+\frac{3}{4} \beta^{2}\right)-\frac{3}{4} \hat{\beta}^{2} \cos 2 \tau\right] \mu^{2}+\left[\left(15 \theta_{1}^{4}-\frac{1}{4} \theta_{1}^{2} \beta^{2}+\frac{4}{6} \frac{5}{4} \beta^{4}\right)\right.\right.\right.$

$\left.\left.+\left(\frac{18}{4} \theta_{1}^{2} \beta^{2}-\frac{15}{16} \beta^{4}\right) \cos 2 \tau+\frac{15}{4} \beta^{4} \cos 4 \tau\right] \mu^{4}+\cdots\right\}$

$\left.+\left\{-3+\left[\left(12 \theta_{1}^{2}-3 \beta^{2}\right)+3 \beta^{2} \cos 2 \tau\right] \mu^{2}+\cdots\right\} \rho+\cdots\right\}$

$=\left\{3+\left[\left(-16 \theta_{1}^{2}+6 \beta^{2}\right)-12 \beta^{2} \cos 2 \tau\right] \mu^{2}+\left[\left(90 \theta_{1}^{4}+\frac{59}{4}-\theta_{1}^{2} \beta^{2}+\frac{69}{6} \frac{9}{4} \beta^{4}\right)\right.\right.$

(50)

$\left.\left.+\left(\frac{1}{4} \theta_{1}^{2} \beta^{2}-\frac{6}{16} \frac{3}{6} \beta^{4}\right) \cos 2 \tau+\frac{183}{64} \beta^{4} \cos 4 \tau\right] \mu^{4}+\cdots\right\} \rho^{2}+\{-12 \beta \mu \sin \tau$

$\left.+\left[\left(-90 \theta_{1}^{2} \beta+\frac{45}{4} \beta^{3}\right) \sin \tau-\frac{15}{4} \beta^{3} \sin 3 \tau\right] \mu^{3}+\cdots\right\} \rho \sigma$

$+\left\{\frac{3}{2}+\left[\left(\frac{3}{2} \theta_{1}^{2}-\frac{33}{8} \beta^{2}\right)+\frac{33}{8} \beta^{2} \cos 2 \tau\right] \mu^{2}+\cdots\right\} \sigma^{2}+$

(b) $\sigma^{\prime \prime}+\left\{1+\left[-\frac{3}{2} \beta^{2}+\frac{3}{2} \beta^{2} \cos 2 \tau\right] \mu^{2}+\left[-4 \theta_{1}^{2} \beta^{2}+7 \theta_{1}^{2} \beta^{2} \cos 2 \tau\right] \mu^{4}+\cdots\right\} \sigma$ $+\left\{-3 \beta \mu \sin \tau+\left[\left(-3 \theta_{1}^{2} \beta+\frac{9}{8} \beta^{3}\right) \sin \tau-\frac{3}{8} \beta^{3} \sin 3 \tau\right] \mu^{3}+\cdots\right\} \rho$ $=\left\{-6 \beta \mu \sin \tau+\left[\left(-21 \theta_{1}^{2} \beta+\frac{63}{8} \beta^{3}\right) \sin \tau-\frac{21}{8} \beta^{3} \sin 3 \tau\right] \mu^{3}+\cdots\right\} \rho^{2}$ $+\left\{3+\left[\left(3 \theta_{1}^{2}-\frac{33}{4} \beta^{2}\right)+\frac{33}{4} \beta^{2} \cos 2 \tau\right] \mu^{2}+\cdots\right\} \rho \sigma+\left\{\frac{9}{2} \beta \mu \sin \tau+\cdots\right\} \sigma^{2}+$

In the first of these equations the coefficients of all terms containing odd powers of $\sigma$ involve only odd powers of $\mu$ and sines of odd multiples of $\tau$. All other coefficients involve only even powers of $\mu$ and cosines of even multiples of $\tau$. In the second equation the coefficient of every odd power of $\sigma$ involves only even powers of $\mu$ and cosines of even multiples of $\tau$. All other coefficients involve only odd powers of $\mu$ and sines of odd multiples of $\tau$. These properties play an important rôle throughout the entire discussion.

\section{$\S 12$. The equations of variation.}

Considering merely the linear terms of the differential equations $(50)$ for $\rho$ and $\sigma$ we have the equations of variation:

(a) $\rho^{\prime \prime}+\left\{1+\left[\left(-4 \theta_{1}^{2}+\frac{3}{2} \beta^{2}\right)-\frac{9}{2} \beta^{2} \cos 2 \tau\right] \mu^{2}\right.$

$$
\left.+\left[\left(20 \theta_{1}^{4}+6 \theta_{1}^{2} \beta^{2}+\frac{3}{8} \beta^{4}\right)+\left(\theta_{1}^{2} \beta^{2}-\frac{3}{2} \beta^{4}\right) \cos 2 \tau+\frac{9}{8} \beta^{4} \cos 4 \tau\right] \mu^{4}+\cdots\right\} \rho
$$

$$
+\left\{-3 \beta \mu \sin \tau+\left[\left(-3 \theta_{1}^{2} \beta+\frac{9}{8} \beta^{3}\right) \sin \tau-\frac{3}{8} \beta^{3} \sin 3 \tau\right] \mu^{3}+\cdots\right\} \sigma=0 \text {. }
$$

(b) $\sigma^{\prime \prime}+\left\{1+\left[-\frac{3}{2} \beta^{2}+\frac{3}{2} \beta^{2} \cos 2 \tau\right] \mu^{2}+\cdots\right\} \sigma$

$$
+\left\{-3 \beta \mu \sin \tau+\left[\left(-3 \theta_{1}^{2} \beta+\frac{9}{8} \beta^{3}\right) \sin \tau-\frac{3}{8} \beta^{3} \sin 3 \tau\right] \mu^{3}+\cdots\right\} \rho=0 .
$$


These equations admit a solution of the form *

$$
\begin{aligned}
& \rho_{j}=A_{j} e^{\lambda, \tau} \phi_{j}(\tau), \\
& \sigma_{i}=A_{j} e^{\lambda_{j} \tau} \psi_{i}(\tau)
\end{aligned}
$$$$
(j=1, \cdots, 4)
$$

where $\lambda_{j}$ is a root of the fundamental equation and where $\phi_{j}(\tau)$ and $\psi_{j}(\tau)$ are periodic functions of $\tau$ with the period $2 \pi$. The four values of the $\lambda_{j}$ (real or imaginary) are associated in pairs, equal in value but of opposite sign. $\dagger$ From the fact that $\tau$ does not occur explicitly in the original equations (1) it is known $\grave{a}$ priori that one pair of the $\lambda_{j}$ has the value $0, \ddagger$ and consequently, if we choose the notation so that $\lambda_{3}=\lambda_{4}=0$, the two corresponding solutions will have the forms

$$
\begin{array}{ll}
\rho_{3}=A_{3} \phi_{3}(\tau), & \rho_{4}=A_{4}\left[\phi_{4}(\tau)+\tau \phi_{3}(\tau)\right], \\
\sigma_{3}=A_{3} \psi_{3}(\tau), & \sigma_{4}=A_{4}\left[\psi_{4}(\tau)+\tau \psi_{3}(\tau)\right] .
\end{array}
$$

We shall consider first the two solutions in which the $\lambda_{j}$ are not zero. Let us first substitute in (51) the forms

$$
\begin{array}{ll}
\rho=e^{i \lambda \tau} \phi(\tau) & (i=\sqrt{-1}), \\
\sigma & =e^{i \lambda \tau} \psi(\tau) .
\end{array}
$$

After dividing out the exponential there remains

$$
\begin{aligned}
& \phi^{\prime \prime}+2 i \lambda \phi^{\prime}+\left[1-\lambda^{2}+a_{2} \mu^{2}+a_{4} \mu^{4}+\cdots\right] \phi+\left[a_{1} \mu+a_{3} \mu^{3}+\cdots\right] \psi=0, \\
& \psi^{\prime \prime}+2 i \lambda \psi^{\prime}+\left[1-\lambda^{2}+b_{2} \mu^{2}+b_{4} \mu^{4}+\cdots\right] \psi+\left[a_{1} \mu+a_{3} \mu^{3}+\cdots\right] \phi=0,
\end{aligned}
$$

where

$$
\begin{aligned}
& a_{1}=-3 \beta \sin \tau, \\
& a_{3}=\left(-3 \theta_{1}^{2} \beta+\frac{9}{8} \beta^{3}\right) \sin \tau-\frac{3}{8} \beta^{3} \sin 3 \tau, \\
& a_{2}=\left(-4 \theta_{1}^{2}+\frac{3}{2} \beta^{2}\right)-\frac{9}{2} \beta^{2} \cos 2 \tau, \\
& a_{4}=\left(20 \theta_{1}^{4}+6 \theta_{1}^{2} \beta^{2}+\frac{3}{8} \beta^{4}\right)+\left(\theta_{1}^{2} \beta^{2}-\frac{3}{2} \beta^{4}\right) \cos 2 \tau+\frac{9}{8} \beta^{4} \cos 4 \tau, \\
& b_{2}=-\frac{3}{2} \beta^{2}+\frac{3}{2} \beta^{2} \cos 2 \tau, \\
& b_{4}=\text { a sum of cosines of even multiples of } \tau .
\end{aligned}
$$

With respect to equations (52) it is known that $\phi$ and $\psi$ are periodic with the

* Floquet, Annales Scientifiques de l'École Normale Supérieure, 2d series, vol. 12 (1883), p. 47.

† See Les Méthodes Nouvelles de la Mécanique Céleste, Vol. 1, p. 193.

$\ddagger$ Ibid., p. 187. 
period $2 \pi$, and that $\lambda$ vanishes with $\mu$ since the problem then reduces to the two-body problem in which the characteristic exponents are all zero. It can be shown that $\phi, \psi$ and $\lambda$ are expansible as power series in $\mu$ of the form

$$
\phi=\sum_{j=0}^{\infty} \phi_{j} \mu^{j}, \quad \psi=\sum_{j=0}^{\infty} \psi_{j} \mu^{j}, \quad \lambda=\sum_{j=1}^{\infty} \lambda_{j} \mu^{j} .
$$

Substituting these expressions in (52) we find:

Coefficient of $\mu^{\prime \prime}$.

$$
\begin{aligned}
& \phi_{0}^{\prime \prime}+\phi_{0}=0, \\
& \psi_{0}^{\prime \prime}+\psi_{0}=0 .
\end{aligned}
$$

Therefore

Coefficient of $\mu$.

$$
\begin{aligned}
& \phi_{0}=\alpha_{1}^{(0)} \cos \tau+\alpha_{2}^{(0)} \sin \tau, \\
& \psi_{0}=\gamma_{1}^{(0)} \cos \tau+\gamma_{2}^{(0)} \sin \tau .
\end{aligned}
$$

$$
\begin{aligned}
& \phi_{1}^{\prime \prime}+\phi_{1}=-2 i \lambda_{1} \phi_{0}^{\prime}-a_{1} \psi_{0}, \\
& \psi_{1}^{\prime \prime}+\psi_{1}=-2 i \lambda_{1} \psi_{0}^{\prime}-a_{1} \phi_{0} .
\end{aligned}
$$

Since the periodicity conditions demand that the coefficients of $\cos \tau$ and $\sin \tau$ in the right members be zero, we must take $\lambda_{1}=0$. We get then

$$
\begin{aligned}
& \phi_{1}^{\prime \prime}+\phi_{1}=\frac{3}{2} \beta \gamma_{2}^{(0)}-\frac{3}{2} \beta \gamma_{2}^{(0)} \cos 2 \tau+\frac{3}{2} \beta \gamma_{1}^{(0)} \sin 2 \tau, \\
& \psi_{1}^{\prime \prime}+\psi_{1}={ }_{2}^{3} \beta \alpha_{2}^{(())}-\frac{3}{2} \beta \alpha_{2}^{(0)} \cos 2 \tau+\frac{3}{2} \beta \alpha_{1}^{(0)} \sin 2 \tau .
\end{aligned}
$$

Integrating we have

$$
\begin{aligned}
& \phi_{1}=\alpha_{1}^{(1)} \cos \tau+\alpha_{2}^{(1)} \sin \tau+\frac{3}{2} \beta \gamma_{2}^{(0)}+\frac{1}{2} \beta \gamma_{2}^{(0)} \cos 2 \tau-\frac{1}{2} \beta \gamma_{1}^{(0)} \sin 2 \tau, \\
& \psi_{1}=\gamma_{1}^{(1)} \cos \tau+\gamma_{2}^{(1)} \sin \tau+\frac{3}{2} \beta \alpha_{2}^{(0)}+\frac{1}{2} \beta \alpha_{2}^{(0)} \cos 2 \tau-\frac{1}{2} \beta \alpha_{1}^{(0)} \sin 2 \tau .
\end{aligned}
$$

Coefficient of $\mu^{2}$.

or, expanded,

$$
\begin{aligned}
\phi_{2}^{\prime \prime}+\phi_{2} & =-2 i \lambda_{2} \phi_{0}^{\prime}-a_{2} \phi_{1}-a_{1} \psi_{1}, \\
\psi_{2}^{\prime \prime}+\psi_{2} & =-2 i \lambda_{2} \psi_{0}^{\prime}-b_{2} \psi_{0}-a_{1} \phi_{1}
\end{aligned}
$$

$$
\begin{gathered}
\phi_{2}^{\prime \prime}+\phi_{2}={ }_{2}^{3} \beta \gamma_{2}^{(1)}-{ }_{2}^{3} \beta \gamma_{2}^{(1)} \cos 2 \tau+{ }_{2}^{3} \gamma_{1}^{(1)} \sin 2 \tau+\left(4 \theta_{1}^{2} \alpha_{2}^{(0)}+2 i \lambda_{2} \alpha_{1}^{(0)}\right) \sin \tau \\
+\left(4 \theta_{1}^{2} \alpha_{1}^{(0)}-2 i \lambda_{2} \alpha_{2}^{(0)}\right) \cos \tau+3 \beta^{2} \alpha_{2}^{(0)} \sin 3 \tau+3 \beta^{2} \alpha_{1}^{(0)} \cos 3 \tau \\
\psi_{2}+\psi_{2}=\frac{3}{2} \beta \alpha_{2}^{(1)}+\left(\frac{15}{2}-\beta^{2} \gamma_{2}^{(0)}+2 i \lambda_{2} \gamma_{1}^{(0)}\right) \sin \tau+2 i \lambda_{2} \gamma_{2}^{(0)} \cos \tau \\
+{ }_{2}^{3} \beta \alpha_{1}^{(1)} \sin 2 \tau-{ }_{2}^{3} \beta \alpha_{2}^{(1)} \cos 2 \tau
\end{gathered}
$$


In order to satisfy the periodicity condition we must have

$$
\begin{aligned}
2 i \lambda_{2} \alpha_{1}^{(0)}+4 \theta_{1}^{2} \alpha_{2}^{(0)} & =0, & 2 i \lambda_{2} \gamma_{1}^{(0)}+{ }_{2}^{15} \beta^{2} \gamma_{2}^{(0)} & =0, \\
4 \theta_{1}^{2} \alpha_{1}^{(0)}-2 i \lambda_{2} \alpha_{2}^{(0)} & =0, & +2 i \lambda_{2} \gamma_{2}^{(0)} & =0 .
\end{aligned}
$$

The equations of the second column are satisfied by taking

$$
\gamma_{1}^{(0)}=\gamma_{2}^{(0)}=0 \text {. }
$$

Solving the other two we find

$$
\lambda_{2}= \pm 2 \theta_{1}^{2}, \quad \alpha_{1}^{(0)}-i a_{2}^{(0)}=0 .
$$

Equations (59) can also be satisfied by taking

$$
\lambda_{2}=\alpha_{1}^{(0)}=\alpha_{2}^{(0)}=\gamma_{2}^{(0)}=0, \quad \gamma_{1}^{(0)}=\text { arbitrary },
$$

but this would lead to the development of the solutions in which the characteristic exponent is zero, and these solutions will be discussed later.

It was known at the outset that there were two values of $\lambda$ equal numerically but of opposite sign. We will choose the one with the positive sign. The solution for the negative $\lambda$ can be derived from the solution for the positive $\lambda$. The condition $\alpha_{1}^{(0)}-i \alpha_{2}^{(0)}=0$ still leaves us with an arbitrary constant. Since the equations are linear this constant will enter the solution linearly and may therefore be taken equal to unity. The arbitrary constant is restored in (79) after the solutions are completely developed. We will take then $\alpha_{1}^{(0)}=1$ which makes $\alpha_{2}^{(0)}=-i$. Consequently

$$
\phi_{0}=\cos \tau-i \sin \tau, \quad \psi_{0}=0 .
$$

Integrating (58) with these values, we get

$$
\begin{array}{r}
\phi_{2}={ }_{2}^{3} \beta \gamma_{2}^{(1)}+\alpha_{1}^{(2)} \cos \tau+\alpha_{2}^{(2)} \sin \tau+\frac{1}{2} \beta \gamma_{2}^{(1)} \cos 2 \tau-\frac{1}{2} \beta \gamma_{1}^{(1)} \sin 2 \tau \\
+3 \beta^{2} \cos 3 \tau-3 \beta^{2} i \sin 3 \tau,
\end{array}
$$

$$
\psi_{2}=\frac{3}{2} \beta a_{2}^{(1)}+\gamma_{1}^{(2)} \cos \tau+\gamma_{2}^{(2)} \sin \tau+\frac{1}{2} \beta \alpha_{2}^{(1)} \cos 2 \tau-\frac{1}{2} \beta \alpha_{1}^{(1)} \sin 2 \tau .
$$

Coefficient of $\mu^{3}$.

$$
\begin{aligned}
& \phi_{3}^{\prime \prime}+\phi_{3}=\frac{3}{2} \beta \gamma_{2}^{(2)}+\left[-2 \lambda_{3}+4 \theta_{1}^{2}\left(\alpha_{1}^{(1)}-i \alpha_{2}^{(1)}\right)\right] \cos \tau \\
&+\left[2 i \lambda_{3}+4 \theta_{1}^{2}\left(\alpha_{2}^{(1)}+i \alpha_{1}^{(1)}\right)\right] \sin \tau+\frac{3}{2} \beta \gamma_{1}^{(2)} \sin 2 \tau-\frac{3}{2} \beta \gamma_{2}^{(2)} \cos 2 \tau, \\
&(62) \psi_{3}^{\prime \prime}+\psi_{3}= {\left[\frac{3}{2} \alpha_{2}^{(2)} \beta-\frac{3}{2} i \theta_{1}^{2} \beta-\frac{2}{1} \frac{1}{6} i \beta^{3}\right]+\left[-2 i \lambda_{2} \gamma_{2}^{(1)}+\frac{3}{4} \beta^{2} \gamma_{1}^{(1)}\right] \cos \tau } \\
&+\left[2 i \lambda_{2} \gamma_{1}^{(1)}+\frac{9}{4} \beta^{2} \gamma_{2}^{(1)}\right] \sin \tau+\left[-\frac{3}{2} \alpha_{2}^{(2)} \beta+\frac{1}{2} i \theta_{1}^{2} \beta+\frac{21}{16} i \beta^{3}\right] \cos 2 \tau \\
&+\left[\frac{3}{2} \alpha_{1}^{(2)} \beta+\frac{11}{2} \theta_{1}^{2} \beta-\frac{9}{16} \beta^{3}\right] \sin 2 \tau-\frac{3}{4} \beta^{2} \gamma_{1}^{(1)} \cos 3 \tau-\frac{3}{4} \beta^{2} \gamma_{2}^{(1)} \sin 3 \tau
\end{aligned}
$$


From the periodicity conditions we must have

$$
\begin{aligned}
-2 \lambda_{3}+4 \theta_{1}^{2}\left(\alpha_{1}^{(1)}-i \alpha_{2}^{(1)}\right) & =0, & & \frac{3}{4} \beta^{2} \gamma_{1}^{(1)}-2 i \lambda_{2} \gamma_{2}^{(1)}=0, \\
2 i \lambda_{3}+4 \theta_{1}^{2}\left(i \alpha_{1}^{(1)}+\alpha_{2}^{(1)}\right) & =0, & & 2 i \lambda_{2} \gamma_{1}^{(1)}+\frac{9}{4} \beta^{2} \gamma_{2}^{(1)}=0 .
\end{aligned}
$$

The last two equations can be satisfied only if $\gamma_{1}^{(1)}=\gamma_{2}^{(1)}=0$. The first two can be satisfied only if $\lambda_{3}=\left(\alpha_{1}^{(1)}-i \alpha_{2}^{(1)}\right)=0$. The condition $\left(\alpha_{1}^{(1)}-i \alpha_{2}^{(1)}\right)=0$ again gives us an arbitrary constant. Then by (56) $\phi_{1}=c(\cos \tau-i \sin \tau)$, but this is the same as $\phi_{0}$ multiplied by $c \mu$. That is, the solution is repeating itself one degree higher in $\mu$ and this, of course, should be expected since the equations are linear, so that any solution multiplied by any power of $\mu$ must satisfy them. We are at liberty then to choose the arbitrary $c=0$, which is the same as choosing $\alpha_{1}^{(1)}=\alpha_{2}^{(1)}=0$. Integrating (62) with these values, we find

$$
\begin{aligned}
\phi_{3}= & \frac{3}{2} \beta \gamma_{2}^{(2)}+\alpha_{1}^{(3)} \cos \tau+\alpha_{2}^{(3)} \sin \tau+\frac{1}{2} \beta \gamma_{2}^{(2)} \cos 2 \tau-\frac{1}{2} \beta \gamma_{1}^{(2)} \sin 2 \tau \\
\Psi_{3}= & {\left[\frac{3}{2} \alpha_{2}^{(2)} \beta-\frac{3}{2} i \theta_{1}^{2} \beta-\frac{2}{16} i \beta^{3}\right]+\gamma_{1}^{(3)} \cos \tau+\gamma_{2}^{(3)} \sin \tau } \\
& +\left[\frac{1}{2} \alpha_{2}^{(2)} \beta-\frac{11}{6} i \theta_{1}^{2} \beta-\frac{7}{16} i \beta^{3}\right] \cos 2 \tau+\left[-\frac{1}{2} \alpha_{1}^{(2)} \beta-\frac{11}{6} \theta_{1}^{2} \beta+\frac{8}{16} \beta^{3}\right] \sin 2 \tau
\end{aligned}
$$

It can be shown by induction at this point that $\phi$ and $\lambda$ involve only even powers of $\mu$, and that $\psi$ is an odd series in $\mu$. Furthermore $\phi$ contains only odd multiples of $\tau$, and $\psi$ only even multiples. Consequently

and all

$$
\gamma_{1}^{(2)}=\gamma_{2}^{(2)}=\alpha_{1}^{(3)}=\alpha_{2}^{(3)}=\gamma_{1}^{(3)}=\gamma_{2}^{(3)}=0,
$$

Coefficient of $\mu^{4}$.

$$
\phi_{2 j+1}=\psi_{2 j}=0
$$

$$
\phi_{4}^{\prime \prime}+\phi_{4}=\left[-2 \lambda_{4}+4 \theta_{1}^{2}\left(\alpha_{1}^{(2)}-i \alpha_{2}^{(2)}\right)-16 \theta_{1}^{4}-10 \theta_{1}^{2} \beta^{2}\right] \cos \tau+\left[2 i \lambda_{4}\right.
$$

$$
\begin{aligned}
& \left.+4 \theta_{1}^{2}\left(i \alpha_{1}^{(2)}+\alpha_{2}^{(2)}\right)+16 i \theta_{1}^{4}\right] \sin \tau+\left[6 \theta_{1}^{2} \beta^{2}+3 \beta^{2} \alpha_{1}^{(2)}+\frac{8}{16} \beta^{4}\right] \cos 3 \tau \\
& +\left[-6 i \theta_{1}^{2} \beta^{2}+3 \beta^{2} \alpha_{2}^{(2)}-\frac{4}{1} \frac{5}{6} i \beta^{4}\right] \sin 3 \tau-\frac{21}{16} \beta^{4} \cos 5 \tau+\frac{21}{16} i \beta^{4} \sin 5 \tau .
\end{aligned}
$$

Therefore, from the periodicity condition, we must have

$$
\begin{aligned}
-2 \lambda_{4}+4 \theta_{1}^{2}\left(\alpha_{1}^{(2)}-i \alpha_{2}^{(2)}\right)-16 \theta_{1}^{4}-10 \theta_{1}^{2} \beta^{2} & =0, \\
2 i \lambda_{4}+4 \theta_{1}^{2}\left(i \alpha_{1}^{(2)}+\alpha_{2}^{(2)}\right)+16 i \theta_{1}^{4} & =0 .
\end{aligned}
$$

Solving these equations, we find

$$
\lambda_{4}=-8 \theta_{1}^{4}-\frac{5}{2} \theta_{1}^{2} \beta^{2}, \quad \alpha_{1}^{(2)}-i \alpha_{2}^{(2)}=\frac{5}{4} \beta^{2} .
$$

In this last equation we can choose $\alpha_{1}^{(2)}={ }_{4}^{5} \beta^{2}$ and $\alpha_{2}^{(2)}=0$. This choice of $\alpha_{1}^{(2)}$ and $\alpha_{2}^{(2)}$ will make the coefficient of $\sin \tau$ in $\phi_{2}$ equal to zero, and since the same thing occurs for each $\phi_{j}$ it is evident that this method of choosing will simplify 
the solution by making the coefficient of $\sin \tau$ equal to zero for all powers of $\mu$. We have then on integrating

$$
\begin{aligned}
& \phi_{4}=\alpha_{4} \cos \tau+\left[-\frac{3}{4} \theta_{1}^{2} \beta^{2}-\frac{63}{128} \beta^{4}\right] \cos 3 \tau+i\left[\frac{3}{4} \theta_{1}^{2} \beta^{2}+\frac{45}{12} \beta^{4}\right] \sin 3 \tau \\
& +{ }_{12} \frac{7}{2} \beta^{4} \cos 5 \tau-{ }_{1} \frac{7}{2} 8 i \beta^{4} \sin 5 \tau, \\
& \psi_{3}=i\left[-\frac{3}{2} \theta_{1}^{2} \beta-\frac{21}{16} \beta^{3}\right]+i\left[-\frac{1}{6} \theta_{1}^{2} \beta-\frac{7}{16} \beta^{3}\right] \cos 2 \tau+\left[-\frac{11}{6} \theta_{1}^{2} \beta-\frac{7}{16} \beta^{3}\right] \sin 2 \tau \text {, } \\
& \phi_{2}=\frac{5}{4} \beta^{2} \cos \tau-\frac{3}{8} \beta^{2} \cos 3 \tau+\frac{3}{8} i \beta^{2} \sin 3 \tau \text {, } \\
& \psi_{1}=-\frac{3}{2} i \beta-\frac{1}{2} i \beta \cos 2 \tau-\frac{1}{2} \beta \sin 2 \tau \text {, } \\
& \phi_{0}=\cos \tau-i \sin \tau \text {, } \\
& \lambda=2 \theta_{1}^{2} \mu^{2}+\left(-8 \theta_{1}^{4}-\frac{5}{2} \theta_{1}^{2} \beta^{2}\right) \mu^{4}+\cdots
\end{aligned}
$$

The coefficient $\alpha_{4}$ of $\cos \tau$ in $\phi_{4}$ is determined by the periodicity condition on $\phi_{6}$. That the process of determining the values of the $\lambda_{j}$ and the constants of integration arising at each step is general may be shown as follows. Let us suppose that we have computed everything up to and including $\phi_{j}$ with the exception of the constants of integration in $\phi_{i}$. We have then

$$
\phi_{j}=\alpha_{1}^{(j)} \cos \tau+\alpha_{2}^{(j)} \sin \tau+\text { known terms. }
$$

The $\alpha_{1}^{(j)}$ and $\alpha_{2}^{(j)}$ enter $\psi_{j+1}$ as follows :

$$
\begin{aligned}
\psi_{j+1}^{\prime \prime}+\psi_{j+1} & =3 \beta \sin \tau \cdot \phi_{j}+\text { known terms, } \\
& ={ }_{2}^{3} \beta \alpha_{2}^{(j)}-\frac{3}{2} \beta \alpha_{2}^{(j)} \cos 2 \tau+\frac{3}{2} \beta \alpha_{1}^{(j)} \sin 2 \tau+\text { known terms. }
\end{aligned}
$$

Consequently in so far as it involves $\alpha_{1}^{(j)}$ and $\alpha_{2}^{(j)}$

$$
\psi_{j+1}={ }_{2}^{3} \beta \alpha_{2}^{(j)}+\frac{1}{2} \beta \alpha_{2}^{(j)} \cos 2 \tau-\frac{1}{2} \beta \alpha_{1}^{(j)} \sin 2 \tau .
$$

Similarly, in so far as $\phi_{j+2}$ depends upon constants as yet undetermined

$$
\begin{aligned}
\phi_{j+2}^{\prime \prime}+\phi_{j+2} & =-2 i \lambda_{2} \phi_{j}^{\prime}-2 i \lambda_{j+2} \phi_{0}^{\prime}+\left[\left(4 \theta_{1}^{2}-\frac{3}{2} \beta^{2}\right)+\frac{9}{2} \beta^{2} \cos 2 \tau\right] \phi_{j}+3 \beta \sin \tau \psi_{j+1} \\
& =\left[-2 \lambda_{j+2}+4 \theta_{1}^{2}\left(\alpha_{1}^{(j)}-i \alpha_{2}^{(j)}\right)+A_{j+2}\right] \cos \tau \\
& +\left[2 i \lambda_{j+2}+4 \theta_{1}^{2}\left(i \alpha_{1}^{(j)}+\alpha_{2}^{(j)}\right)+B_{j+2}\right] \sin \tau \\
& +3 \beta^{2} \alpha_{1}^{(j)} \cos 3 \tau+3 \beta^{2} \alpha_{2}^{(j)} \sin 3 \tau,
\end{aligned}
$$

where $A_{j+2}$ and $B_{j+2}$ are the known terms in the coefficients of $\cos \tau$ and $\sin \tau$ respectively. From the periodicity condition we must have

$$
\begin{aligned}
& -2 \lambda_{j+2}+4 \theta_{1}^{2}\left(\alpha_{1}^{(j)}-i \alpha_{2}^{(j)}\right)+A_{j+2}=0, \\
& +2 i \lambda_{j+2}+4 \theta_{1}^{2}\left(i \alpha_{1}^{(j)}+\alpha_{2}^{(j)}\right)+B_{j+2}=0 .
\end{aligned}
$$


The solution of these equations is

$$
\begin{aligned}
\lambda_{j+2} & =\frac{1}{4}\left(A_{j+2}+i B_{j+2}\right), \\
\alpha_{1}^{(1)}-i \alpha_{2}^{(j)} & =-\frac{A_{j+2}-i B_{j+2}}{8 \theta_{1}^{2}} .
\end{aligned}
$$

As has already been pointed out we can choose $\alpha_{1}^{(j)}=0$ and we have then

$$
\alpha_{i}^{(j)}=-\frac{A_{j+2}-i B_{j+2}}{8 \theta_{1}^{2}}
$$

In order to show that $\lambda_{j+2}$ and $\alpha_{1}^{(j)}$ are real it will be sufficient to show that $A_{j+2}$ is real and $B_{j+2}$ is a pure imaginary. This is readily shown by induction, for up to $j=4$ inclusive we have

$$
\begin{aligned}
& \phi_{j}=\sum_{\kappa} m_{\kappa} \cos \kappa \tau+i \sum_{\kappa} n_{\kappa} \sin \kappa \tau, \\
& \psi_{j}=i \sum_{\kappa} . f_{\kappa} \cos \kappa \tau+\sum_{\kappa} g_{\kappa} \sin \kappa \tau,
\end{aligned}
$$

where $m_{\kappa}, n_{\kappa}, f_{\kappa}$ and $g_{\kappa}$ are all real. From the form of the differential equations it follows at once that the same forms hold for $j=5$, then $j=6$, and so ou. That is $A_{j+2}$ is real while $B_{j+2}$ is a pure imaginary.

It is further to be noticed that $A_{j+2}$ and $B_{j+2}$ do not contain any terms in $\beta$ independent of $\theta_{1}^{2}$, and consequently the $\theta_{1}^{2}$ which appears in the denominator of $\alpha_{1}^{(j)}$ will divide out. This is proved as follows. If $\theta_{1}^{2}$ be put equal to zero in the differential equations then equations (52) become the equations of variation of a circular orbit in the ordinary two-body problem, the plane of the circle being inclined to the plane of reference by an angle whose sine is $\beta \mu=s$. The original differential equations $(6)$ can then be written

$$
r^{\prime \prime}=\frac{\left(1-s^{2}\right)\left(1-e^{2}\right)}{r^{3}}-\frac{r}{\left(r^{2}+q^{2}\right)^{\frac{3}{2}}}=R, \quad q^{\prime \prime}=-\frac{q}{\left(r^{2}+q^{2}\right)^{\frac{3}{3}}}=Q
$$

where the constant $c^{2}$ is given the form $\left(1-s^{2}\right)\left(1-e^{2}\right)$. For these equations we have the solution

$$
\begin{aligned}
& r=a \frac{\left(1-e^{2}\right) \sqrt{1-s^{2} \sin ^{2}(\theta-\Omega)}}{1+e \cos \left(\theta-\theta_{0}\right)}, \\
& q=a \frac{\left(1-e^{2}\right) s \sin (\theta-\Omega)}{1+e \cos \left(\theta-\theta_{0}\right)},
\end{aligned}
$$

where

$$
\left(\theta-\theta_{0}\right)=\left(\tau-\tau_{0}\right)+2 e \sin \left(\tau-\tau_{0}\right)+\cdots
$$


Let us now form the equations of variation by putting

$$
r=r_{0}+\rho, \quad q=q_{0}+\sigma, \quad e=e_{0}+\epsilon,
$$

where $r_{0}, q_{0}$ and $e_{0}$ are the values in (71). We find

$$
\begin{aligned}
& \rho^{\prime \prime}=\frac{\partial R}{\partial r} \rho+\frac{\partial R}{\partial q} \sigma+\frac{\partial R}{\partial e} \epsilon \\
& \sigma^{\prime \prime}=\frac{\partial Q}{\partial r} \rho+\frac{\partial R}{\partial q} \sigma+\frac{\partial Q}{\partial e} \epsilon
\end{aligned}
$$

Three solutions of these equations are given by*

$$
\begin{array}{lll}
\rho=c_{1} \frac{\partial r_{0}}{\partial \Omega}, & \rho=c_{2} \frac{\partial r_{0}}{\partial \tau_{0}}, & \rho=c_{3} \frac{\partial r_{0}}{\partial e_{0}}, \\
\sigma=c_{1} \frac{\partial q_{0}}{\partial \Omega}, & \sigma=c_{2} \frac{\partial q_{0}}{\partial \tau_{0}}, & \sigma=c_{3} \frac{\partial q_{0}}{\partial e_{0}}, \\
\epsilon=c_{1} \frac{\partial e_{0}}{\partial \Omega}, & \epsilon=c_{2} \frac{\partial e_{0}}{\partial \tau_{0}}, & \epsilon=c_{3} \frac{\partial e_{0}}{\partial e_{0}} .
\end{array}
$$

If $e_{0} \neq 0$ these three solutions are distinct, but the case in which we are interested is when $e_{0}=0$. In this case it is not difficult to see that the first two solutions coincide. Since the equations are linear, the system

$$
\begin{aligned}
& \rho=c_{4}\left[\frac{\partial r_{0}}{\partial \Omega}-\frac{\partial r_{0}}{\partial \tau_{0}}\right], \\
& \sigma=c_{4}\left[\frac{\partial q_{0}}{\partial \Omega}-\frac{\partial q_{0}}{\partial \tau_{0}}\right], \\
& \epsilon=c_{4}\left[\frac{\partial e_{0}}{\partial \Omega}-\frac{\partial e_{0}}{\partial \tau_{0}}\right],
\end{aligned}
$$

is also a solution, but as it vanishes for $e_{0}=0$ it carries $e_{0}$ as a factor. We can divide out this factor and absorb it into the arbitrary $c_{4}$. For $e_{0}=0$ this solution does not now vanish, and it is moreover distinct from the first solution. Thus we have three distinct solutions even when $e_{0}=0$, but since $\partial Q / \partial e_{0} \equiv 0$, and $\partial R / \partial e_{0}$ carries $e_{0}$ as a factor, equations (72) pass over to the equations of variation of a circle when $e_{0}=0$. For these equations we have three solutions which are periodic with the period $2 \pi$. The fourth solution is not periodic but involves a term of the form $\tau$ times a periodic function.

Let us return now to the solution which we have developed, (65), and consider only the terms which belong to the two-body problem, viz., the terms which are

* See Les Méthodes Nouvelles de la Mécanique Céleste, vol. I, p. 163. 
independent of $\theta_{1}^{2}$. This solution may be separated into two solutions, one of which is real, the other a pure imaginary. The real solution is the third solution of (73), and the purely imaginary is the second solution. Since both of these solutions are certainly periodic with the period $2 \pi$, it follows that no term in $\beta$ alone can occur in the $A_{j+2}$ and $B_{j+2}$ of (66) for the presence of such terms would give rise to non-periodic terms in the two-body problem. Hence $A_{j+2}$ and $B_{j+2}$ carry $\theta_{1}^{2}$ as a factor which may be divided out in equation (69). Further $\lambda_{j+2},(68)$, carries $\theta_{1}^{2}$ as a factor and therefore $\lambda$ vanishes with the oblateness of the spheroid.

The solution (65) may now be written

where

$$
\begin{aligned}
& \rho^{(1)}=e^{i \lambda \tau}\left[\phi^{(1)}+i \phi^{(2)}\right], \\
& \sigma^{(1)}=e^{i \lambda \tau}\left[\psi^{(1)}+i \psi^{(2)}\right],
\end{aligned}
$$

$$
\begin{aligned}
& \phi^{(1)}=\cos \tau+\left[\frac{5}{4} \beta^{2} \cos \tau-\frac{3}{8} \beta^{2} \cos 3 \tau\right] \mu^{2} \\
& +\left[\alpha_{4} \cos \tau+\left(-\frac{3}{4} \theta_{1}^{2} \beta^{2}-\frac{63}{128} \beta^{4}\right) \cos 3 \tau+{ }_{1}^{\frac{7}{2} 8} \beta^{4} \cos 5 \tau\right] \mu^{4}+\cdots, \\
& \phi^{(2)}=-\sin \tau+\left[\frac{3}{8} \beta^{2} \sin \delta \tau\right] \mu^{2} \\
& +\left[\left(\frac{3}{4} \theta_{1}^{2} \beta^{2}+\frac{45}{128} \beta^{4}\right) \sin 3 \tau-\frac{7}{12} \beta_{8}^{4} \sin 5 \tau\right] \mu^{4}+\cdots, \\
& \psi^{(1)}=\left[-\frac{1}{2} \beta \sin 2 \tau\right] \mu+\left[\left(-\frac{11}{6} \theta_{1}^{2} \beta-\frac{7}{16} \beta^{3}\right) \sin 2 \tau\right] \mu^{3}+\cdots, \\
& \psi^{(2)}=\left[-\frac{3}{2} \beta-\frac{1}{2} \beta \cos 2 \tau\right] \mu \\
& +\left[\left(-\frac{3}{2} \theta_{1}^{2} \beta-\frac{21}{16} \beta^{3}\right)+\left(-\frac{11}{6} \theta_{1}^{2} \beta-\frac{7}{16} \beta^{3}\right) \cos 2 \tau\right] \mu^{3}+\cdots
\end{aligned}
$$

By putting

$$
e^{i \lambda \tau}=\cos \lambda \tau+i \sin \lambda \tau
$$

we can write

$$
\begin{aligned}
& \rho^{(1)}=\left[\phi^{(1)} \cos \lambda \tau-\phi^{(2)} \sin \lambda \tau\right]+i\left[\phi^{(2)} \cos \lambda \tau+\phi^{(1)} \sin \lambda \tau\right], \\
& \sigma^{(1)}=\left[\psi^{(1)} \cos \lambda \tau-\psi^{(2)} \sin \lambda \tau\right]+i\left[\psi^{(2)} \cos \lambda \tau+\psi^{(1)} \sin \lambda \tau\right] .
\end{aligned}
$$

We have thus one solution of the differential equations. A second solution can be derived from it by merely changing the sign of $i$. Thus

$$
\begin{aligned}
& \rho^{(2)}=\left[\phi^{(1)} \cos \lambda \tau-\phi^{(2)} \sin \lambda \tau\right]-i\left[\phi^{(2)} \cos \lambda \tau+\phi^{(1)} \sin \lambda \tau\right], \\
& \sigma^{(2)}=\left[\psi^{(1)} \cos \lambda \tau-\psi^{(2)} \sin \lambda \tau\right]-i\left[\psi^{(2)} \cos \lambda \tau+\psi^{(1)} \sin \lambda \tau\right] .
\end{aligned}
$$

By adding and subtracting these two solutions we have finally

$$
\begin{aligned}
& \rho=A\left[\rho^{(1)}+\rho^{(2)}\right]+B\left[\rho^{(1)}-\rho^{(2)}\right], \\
& \sigma=A\left[\sigma^{(1)}+\sigma^{(2)}\right]+B\left[\sigma^{(1)}-\sigma^{(2)}\right],
\end{aligned}
$$


$A$ and $B$ being two arbitrary constants. As above developed there is a certain arbitrariness in these solutions owing to the manner in which the constants of integration were determined. They may be reduced to a normal form by multiplying each solution by the proper power series in $\mu^{2}$ with constant coefficients. By this process we can make for $\tau=0$,

$$
\rho^{(1)}(0)+\rho^{(2)}(0)=1, \quad \sigma^{(1)}(0)-\sigma^{(2)}(0)=\beta \mu .
$$

Since $\left[\rho^{(1)}-\rho^{(2)}\right]$ and $\left[\sigma^{(1)}+\sigma^{(2)}\right]$ are sine series they vanish for $\tau=0$.

The third and fourth solutions of the equations of variation, (51), are given by *

$$
\begin{array}{ll}
\rho_{3}=C \frac{\partial r_{0}}{\partial \tau_{0}}, & \rho_{4}=D^{\prime} \frac{\partial\left(a r_{0}\right)}{\partial a}, \\
\sigma_{3}=C \frac{\partial q_{0}}{\partial \tau_{0}}, & \sigma_{4}=D^{\prime}-\frac{\partial\left(a q_{0}\right)}{\partial a} .
\end{array}
$$

In forming the partial derivatives with respect to $a$ in the fourth solution it should be remembered that $\theta_{1}^{2}$ is an explicit function of $a$, by equations (4), and that $\tau$ is also a function of $a$ implicitly through $n$. The third solution also can be normalized by giving the arbitrary constant such a form that at $\tau=0$

$$
\rho_{3}=0, \quad \sigma_{3}=C \beta \mu .
$$

The fourth solution is non-periodic and has the form

$$
\rho=D\left[\tau \frac{\partial r_{0}}{\partial \tau_{0}}+\phi_{4}\right], \quad \sigma=D\left[\tau \frac{\partial q_{0}}{\partial \tau_{0}}+\psi_{4}\right],
$$

where $\phi_{4}$ and $\psi_{4}$ are periodic functions of $\tau$ with the period $2 \pi$. As in the previous solutions this may be normalized so that at $\tau=0$

$$
\rho_{4}=D \beta^{2} \mu^{2}, \quad \sigma_{4}=0 .
$$

It is also easy to find $\phi_{4}$ and $\psi_{4}$ by substituting (81) in the equations of variation and solving for these variables, which must be periodic.

Carrying out the above operations we find the following general solutions

$$
\begin{aligned}
\rho=A & \left\{\cos (1-\lambda) \tau+\left[-\frac{1}{4} \beta^{2} \cos (1-\lambda) \tau+\frac{5}{8} \beta^{2} \cos (1+\lambda) \tau-\frac{3}{8} \beta^{2} \cos (3-\lambda) \tau\right] \mu^{2}\right. \\
& +\left[\left(-\frac{1}{2} \alpha_{4}+\frac{3}{4} \theta_{1}^{2} \beta^{2}+\frac{2}{3} \frac{1}{2} \beta^{4}\right) \cos (1-\lambda) \tau+\left(\frac{1}{2} \alpha_{4}-\frac{3}{6} \frac{5}{4} \beta^{4}\right) \cos (1+\lambda) \tau\right. \\
& +\left(-\frac{3}{4} \theta_{1}^{2} \beta^{2}-\frac{3}{32} \beta^{4}\right) \cos (3-\lambda) \tau-\frac{9}{12} \frac{1}{8} \beta^{4} \cos (3+\lambda) \tau \\
& \left.\left.+\frac{7}{12} \beta_{8} \beta^{4} \cos (5-\lambda) \tau\right] \mu^{4}+\cdots\right\}
\end{aligned}
$$

* See Les Méthodes Nouvelles de la Mécanique Céleste, vol. 1, p. 163. 


$$
\begin{aligned}
&+B\left\{\frac{1}{2} \sin (1-\lambda) \tau+\left[\left(-\frac{1}{8} \beta^{2}-\frac{5}{6} \theta_{1}^{2}\right) \sin (1-\lambda) \tau-\frac{5}{16} \beta^{2} \sin (1+\lambda) \tau\right.\right. \\
&\left.\left.\quad-\frac{3}{16} \beta^{2} \sin (3-\lambda) \tau\right] \mu^{2} \cdots\right\} \\
&+ C\left\{\left[-\frac{1}{2} \beta^{2} \sin 2 \tau\right] \mu^{2}+\left[\left(\frac{1}{16} \theta_{1}^{2} \beta^{2}-\frac{1}{8} \beta^{4}\right) \sin 2 \tau+\frac{1}{16} \beta^{4} \sin 4 \tau\right] \mu^{4}+\cdots\right\} \\
&+D\left\{\left\{\left[\frac{3}{4} \beta^{2}+\frac{1}{4} \beta^{2} \cos 2 \tau\right] \mu^{2}+\left[\left(\frac{9}{8} \theta_{1}^{2} \beta^{2}-\frac{3}{3} \beta^{4}\right)\right.\right.\right. \\
&\left.\left.+\left(-\frac{9}{8} \theta_{1}^{2} \beta^{2}+\frac{1}{8} \beta^{4}\right) \cos 2 \tau-\frac{1}{3} \frac{1}{2} \beta^{4} \cos 4 \tau\right] \mu^{4} \cdots\right\}+\tau\left\{\left[\frac{3}{4} \beta^{4} \sin 2 \tau\right] \mu^{4}\right. \\
&\left.\left.+\left[\left(\frac{9}{16} \theta_{1}^{2} \beta^{4}+\frac{1}{6} \frac{5}{4} \beta^{6}\right) \sin 2 \tau-\frac{3}{32} \beta^{6} \sin 4 \tau\right] \mu^{6}+\cdots\right\}\right\}, \\
& \sigma=A\left\{\left[\frac{3}{2} \beta \sin \lambda \tau-\frac{1}{2} \beta \sin (2-\lambda) \tau\right] \mu+\left[\frac{3}{2} \theta_{1}^{2} \beta \sin \lambda \tau\right.\right. \\
&\left.\left.\quad-\frac{11}{6} \theta_{1}^{2} \beta \sin (2-\lambda) \tau\right] \mu^{3}+\cdots\right\} \\
&+B\left\{\left[\frac{3}{4} \beta \cos \lambda \tau+\frac{1}{4} \beta \cos (2-\lambda) \tau\right] \mu+\left[-\frac{1}{2} \theta_{1}^{2} \beta \cos \lambda \tau\right.\right. \\
&\left.\left.\quad+\frac{1}{2} \theta_{1}^{2} \beta \cos (2-\lambda) \tau\right] \mu^{3}+\cdots\right\} \\
&+C\left\{[\beta \cos \tau] \mu+[0] \mu^{3}+\cdots\right\} \\
&+D\left\{\left\{\left[\frac{1}{2} \beta \sin \tau\right] \mu+\left[\left(-\frac{7}{4} \theta_{1}^{2} \beta+\frac{1}{2} \beta^{3}\right) \sin \tau\right] \mu^{3}+\cdots\right\}\right. \\
&\left.\quad+\tau\left\{\left[-\frac{3}{2} \beta^{3} \cos \tau\right] \mu^{3}+\left[\left(-\frac{5}{1} \frac{3}{6} \theta_{1}^{2} \beta^{3}+\frac{2}{16} \beta^{5}\right) \cos \tau\right] \mu^{5}+\cdots\right\}\right\} .
\end{aligned}
$$

§13. Non-homogeneous linear differential equations with periodic coefficients.

If we have a set of homogeneous linear differential equations

$$
\frac{d x_{i}}{d t}=\sum_{j=1}^{n} \theta_{i j}(t) x_{j}, \quad(i=1, \cdots, n),
$$

where the $\theta_{i j}$ are periodic functions of $t$ with the period $2 \pi$, we know from the writings of Floquet* that, if the roots of the fundamental equation are all distinct, the solution has the form

$$
x_{i}=\sum_{j=1}^{n} A_{j} e^{u_{j} t} \phi_{i j}(t),
$$

where the $A_{j}$ are arbitrary constants, the $\alpha_{j}$ are constants known as the characteristic exponents, and the $\phi_{i j}$ are periodic functions of $t$ with the period $2 \pi$.

Such equations arise in dynamics whenever we study small variations from a known periodic solution. If we confine our attention entirely to the first powers of the variations the equations are linear and homogeneous, and are known as the "equations of variation." They have been studied extensively by Poincaré in Les Méthodes Nouvelles de la Mécanique Céleste. If the second and higher

*Annales Scientifiques de l'bcole Normale Supérieure, 2d series, vol. 12 (1883), p. 47. 
powers of the variation are considered equations of the same type arise but they are no longer homogeneous. It is necessary for our purpose to derive the character of the solutions of such equations when the non-homogeneous terms are periodic, even though the period be different from $2 \pi$. We will suppose that the equations are the same as (84) with the addition of periodic terms.

\section{Case I.}

We will assume first that the period of the non-homogenous terms is $2 \pi$. The equations are then

$$
\frac{d x_{i}}{d t}=\sum_{j=1}^{n} \theta_{i j} x_{j}+g_{i}(t) \quad(i=1, \cdots, n),
$$

where $\theta_{i j}(t)$ and $g_{i}(t)$ are periodic with the period $2 \pi$. The solutions of (86) may be written

$$
x_{i}=\sum_{j=1}^{n} A_{j} e^{a_{j} t} \phi_{i j}(t)+\psi_{i}(t) \quad(i=1, \cdots, n) .
$$

If in the differential equations we change $t$ into $t+2 \pi$ the equations remain unchanged, but the solutions become

$$
x_{i}=\sum_{j=1}^{n} A_{j} e^{a_{j}(t+2 \pi)} \phi_{i j}(t+2 \pi)+\psi_{i}(t+2 \pi) .
$$

Therefcre equations (88) are also solutions of (86), and consequently

also

$$
\frac{d}{d t} \psi_{i}(t+2 \pi)=\sum_{j=1}^{n} \theta_{i j} \psi_{j}(t+2 \pi)+g_{i}(t)
$$

$$
\frac{d}{d t} \psi_{i}(t)=\sum_{j=1}^{n} \theta_{i j} \psi_{j}(t)+g_{i}(t)
$$

Forming the difference of these equations we find

(89) $\frac{d}{d t}\left[\psi_{i}(t+2 \pi)-\psi_{i}(t)\right]=\sum_{j=1}^{n} \theta_{i j}\left[\psi_{j}(t+2 \pi)-\psi_{j}(t)\right] \quad(i=1, \cdots, n)$.

These equations are the same as (84) and their solutions have the same form as (85), that is

$$
\psi_{i}(t+2 \pi)-\psi_{i}(t)=\sum_{j=1}^{n} B_{j} e^{a, t} \phi_{i j}(t) .
$$

The constants $B_{j}$ in this case are not arbitrary but depend upon the differential equations. They may or may not be zero. Equations (90) may be interpreted as showing that $\psi_{i}(t)$ is composed of two parts, a periodic part and a non- 
periodic part. We may express it therefore in the form

$$
\psi_{i}(t)=\omega_{i}(t)+\sum_{j=1}^{n} B_{j} e^{a, t} \phi_{i j}(t) f_{i j}^{\prime}(t),
$$

where $\omega_{i}(t)$ are periodic with the period $2 \pi$, and the $f_{i j}(t)$ are functions which must be determined. Changing $t$ into $t+2 \pi$ in equations (91) and forming the difference $\psi_{i}(t+2 \pi)-\psi_{i}(t)$, we find

$$
\begin{aligned}
\psi_{i}(t+2 \pi)-\psi_{i}(t) & =\sum_{j=1}^{n} B_{j} e^{a_{j} t} \phi_{i j}(t)\left[e^{2 a_{j} \pi} f_{i j}(t+2 \pi)-f_{i j}(t)\right], \\
& =\sum_{j=1}^{n} B_{j} e^{a_{j} t} \phi_{i j}(t) \quad \text { from equations }(90) .
\end{aligned}
$$

Comparing the coefficients in these equalities we see that

$$
e^{2 a j \pi} f_{i j}(t+2 \pi)-f_{i j}(t)=1,
$$

from which we can determine the character of the functions $f_{i j}(t)$.

For this purpose let us define a new function $\lambda_{i j}(t)$ such that

$$
\lambda_{i j}(t)=e^{a_{j} t} f_{i j}(t)+\frac{e^{a_{j} t}}{1-e^{2 a_{j} \pi}} .
$$

Then by virtue of the relations (93) the $\lambda_{i j}(t)$ are periodic with the period $2 \pi$, for we have

$$
\begin{aligned}
\lambda_{i j}(t+2 \pi) & =e^{a_{j} t} \cdot e^{2 a_{j} \pi} f_{i j}(t+2 \pi)+\frac{e^{a_{j} t} \cdot e^{2 a_{j} \pi}}{1-e^{2 a_{j} \pi}}, \\
& =e^{a_{j} t}\left[f_{i j}(t)+1\right]+\frac{e^{a_{j} t} \cdot e^{2 a_{j} \pi}}{1-e^{2 a_{j} \pi}}, \\
& =e^{a_{j} t} f_{i j}(t)+\frac{e^{a_{j} t}}{1-e^{2 a_{j} \pi}}, \\
& =\lambda_{i j}(t) .
\end{aligned}
$$

On solving (94) we find

$$
f_{i j}(t)=e^{-a_{j} t} \lambda_{i j}(t)+\frac{1}{e^{2 a_{j} \pi}-1},
$$

where the $\lambda_{i j}$ are periodic with the period $2 \pi$. This expression for the $f_{i j}(t)$ substituted in (91) gives

$$
\psi_{i}(t)=\omega_{i}(t)+\sum_{j=1}^{n} B_{j} \phi_{i j} \lambda_{i j}+\sum_{j=1}^{n} \frac{B_{j}}{e^{2 a_{j} \pi}-1} e^{a_{j} t} \phi_{i j}(t) .
$$

The terms included under the last summation sign are merely terms of the complementary function. All the other terms are periodic with the period $2 \pi$. 
This form for the $\psi_{i}(t)$ fails however if for any $j$

that is, if

$$
e^{2 a j \pi}=1
$$

$$
\alpha_{j} \equiv 0 \bmod \sqrt{-1}
$$

In this event equations (93) for such values of $j$ become

$$
f_{i j}(t+2 \pi)-f_{i j}(t)=1 \text {. }
$$

We will define the corresponding value of $\lambda_{i j}$ by the relation

$$
\lambda_{i j}(t)=f_{i j}(t)-\frac{t}{2 \pi} .
$$

By virtue of $(97) \lambda_{i j}$ is periodic with the period $2 \pi$, for

$$
\begin{aligned}
\lambda_{i j}(t+2 \pi) & =f_{i j}(t+2 \pi)-\frac{t+2 \pi}{2 \pi}, \\
& =f_{i j}(t)-\frac{t}{2 \pi}, \\
& =\lambda_{i j}(t) .
\end{aligned}
$$

Solving (98) for $f_{i j}(t)$, we obtain

$$
f_{i j}(t)=\lambda_{i j}(t)+\frac{t}{2 \pi}
$$

Substituting this expression in (91), we find

$$
\psi_{i}(t)=\text { periodic terms }+\frac{t}{2 \pi} \sum_{j} B_{j} e^{a, t} \phi_{i j}(t),
$$

the summation in the last term to be extended over all $j$ such that $\alpha_{j} \equiv 0$ $\bmod \sqrt{-1}$.

We have then the general expression for the solution

$$
x_{i}=\sum_{j=1}^{n} A_{j} e^{a_{j} t} \phi_{i j}(t)+\text { periodic terms }+\frac{t}{2 \pi} \sum_{j,} B_{j} e^{a_{j} t} \phi_{i j}(t),
$$

where the summation in the last term is to be extended over all $j$ such that $\alpha_{j} \equiv 0 \bmod \sqrt{-1}$.

This result may be stated thus :

Theorem I. If the $\theta_{i j}(t)$ and $g_{i}(t)$ are periodic with the period $2 \pi$ and if the characteristic exponents $\alpha_{j}$ are distinct and none of them congruent to zero $\bmod \sqrt{ }-\overline{1}$, then the particular solution is periodic with the period $2 \pi$. If the $\alpha_{j}$ are distinct but some of them congruent to zero $\bmod \sqrt{-1}$, then the particular solution may involve terms of the form $t$ times the corresponding complementary function. 


\section{Case II.}

We will suppose that the $g_{i}(t)$ are periodic and that the period is different from $2 \pi$. We will suppose further that $g_{i}(t)$ are expressible in the form

$$
g_{i}(t)=\sum_{j}\left[a_{i j} \cos (j+\beta) t+b_{i j} \sin (j+\beta) t\right]=e^{\sqrt{-1} \beta t} f_{i}^{(1)}(t)+e^{-\sqrt{-1} \beta t} f_{i}^{(2)}(t),
$$

where $f_{i}^{(1)}(t)$ and $f_{i}^{(2)}(t)$ are periodic with the period $2 \pi$ and $\beta$ is any real number not an integer. The differential equations may then be written

$$
\frac{d x_{i}}{d t}=\sum_{j=1}^{n} \theta_{i j} x_{j}+e^{\sqrt{-1} \beta t} f_{i}^{(1)}(t)+e^{-\sqrt{-1} \beta t} f_{i}^{(2)}(t) .
$$

Since the equations are linear we can consider the particular solutions depending upon $e^{\sqrt{-1} \beta t}$ and $e^{-\sqrt{-1} \beta t}$ separately. The complete solution will be the sum of the two. Let us consider first

$$
\frac{d x_{i}}{d t}=\sum_{j=1}^{n} \theta_{i j} x_{j}+e^{\sqrt{-1} \beta t} f_{i}^{(1)}(t) .
$$

If we make the substitution

$$
x_{i}=e^{\sqrt{-1} \beta t} y_{i}
$$

equations (104) become after dividing out the exponential

$$
\frac{d y_{i}}{d t}+\sqrt{-1} y_{i}=\sum_{j=1}^{n} \theta_{i j} y_{j}+f_{i}^{(1)}(t)
$$

Since $\theta_{i j}$ and $f_{i}^{(1)}$ are periodic with the period $2 \pi$ the discussion of the character of the $y_{i}$ reduces to Case I. The characteristic exponents of equations (105) are $\left(\alpha_{j}-\sqrt{-1} \beta\right)$, where the $\alpha_{j}$ are the characteristic exponents for the homogeneous equations (81). Applying Theorem I we conclude that the $y_{i}$ are periodic with the period $2 \pi$ provided

$$
\alpha_{j} \neq \sqrt{-1} \beta \bmod \sqrt{-1}
$$

$$
(j=1, \cdots, n)
$$

If, however, for any value of $j$

$$
\alpha_{j} \equiv \sqrt{-1} \beta \bmod \sqrt{-1}
$$

then, in general, non-periodic terms will arise just as in Case 1.

The discussion for the second part of the differential equations

$$
\frac{d x_{i}}{d t}=\sum_{j=1}^{n} \theta_{i j} x_{j}+e^{-v-1 \beta t} f_{i}^{(2)}(t)
$$

shows similarly that the particular integral is periodic if

$$
\alpha_{j} \neq-\sqrt{ }-1 \beta \quad(j=1, \cdots, n) .
$$


In equations arising in dynamics the characteristic exponents $\alpha_{j}$ enter in pairs, equal but of opposite sign. Consequently for such equations this last condition

does not differ from

$$
\alpha_{j}=\sqrt{\overline{-1} \beta} \bmod \sqrt{-1} \quad(j=1, \cdots, n),
$$

$$
\alpha_{j}=1 \sqrt{-1} \beta \quad \bmod \sqrt{-1} \quad(j=1, \cdots, n) .
$$

We have then the following

Theorem II. If the $\theta_{i j}$ are periodic with the period $2 \pi$, and if the $g_{i}(t)$ have the form

$$
g_{i}(t)=\sum_{i}\left[a_{i k} \cos (k+\beta) t+b_{i k} \sin (k+\beta) t\right],
$$

and if the characteristic exponents $\alpha_{j}$ are distinct and none of them congruent to $\pm \sqrt{-1} \beta \bmod \sqrt{-1}$, then the particular solution has the form

$$
x_{i}=\sum_{m}\left[c_{i m} \cos (m+\beta) t+d_{i m} \sin (m+\beta) t\right] .
$$

If any of the $\alpha_{j}$ are congruent to $\pm \sqrt{-1} \beta \bmod \sqrt{-1}$, then the particular solution will, in general, contain, in addition to periodic terms, terms of the form $t$ times the corresponding complementary function.

Let us suppose that one of the $\alpha_{j}$ is congruent to $\sqrt{-1} \beta \bmod \sqrt{-1}$. Then, in general, the solution will contain non-periodic terms. If now the $g_{i}(t)$ contain also terms of the form ${ }_{\cos }^{\sin }(p+q \beta) t$, where $p$ and $q$ are integers, these terms will not, in general, give rise to non-periodic terms in the solution, for the $\alpha_{j}$ will not, in general, be congruent to $\sqrt{-1} q \beta$. Indeed, if $\beta$ is not rational, for no integral value of $q$ except unity will $\sqrt{-1} q \beta \equiv \alpha_{j}$. But if $\beta$ (and therefore $\alpha_{j}$ also) is rational, then certain values of $q$ do exist for which $\alpha_{j} \equiv \sqrt{-1} q \beta$. Let us set

$$
\alpha=\frac{i}{j} \sqrt{-1}, \quad \beta=\frac{I}{J}
$$

where $i$ and $j$ are integers relatively prime, and similarly for $I$ and $J$. By hypothesis

$$
\frac{i}{j}-\frac{I}{J} \equiv 0 \bmod 1
$$

then

$$
\frac{i}{j}-q \frac{I}{J} \neq 0 \quad \bmod 1
$$

unless $q=1+l J$ ( $l$ an integer $)$. In this event a term of the form 
${ }_{\sin }^{\cos }(p+q \beta) t$ can also be written ${ }_{\sin }^{\cos }(r+\beta) t(r$ an integer $)$ which involves only the first multiple of $\beta$, and the solution in general involves non-periodic terms.

\section{Case III.}

When two of the characteristic exponents of the homogeneous set are equal the preceding arguments are not applicable. Let us suppose that the homogeneous equations are

$$
\frac{d x_{i}}{d t}=\sum_{j=1}^{n} \theta_{i j} x_{j} \quad(i=1, \cdots, n),
$$

but that $\alpha_{n-1}=\alpha_{n}$. The solution then has in general the form

$$
x_{i}=\sum_{j=1}^{n-2} C_{j} e^{a_{j} t} \phi_{i j}(t)+[A+B t] e^{a_{n} t} \phi_{i n}(t)+B e^{a_{n} t} \phi_{i(n-1)}(t) .
$$

Suppose now the given equations are

$$
\frac{d x_{i}}{d t}=\sum_{j=1}^{n} \theta_{i j} x_{j}+g_{i}(t)
$$

where the $\theta_{i j}$ are the same as in (107) and the $g_{i}$ are periodic functions of $t$ with the period $2 \pi$. Let the particular solutions which depend on the $g_{i}(t)$ be

Just as in Case I we find

$$
x_{i}=\psi_{i}(t) \text {. }
$$

$$
\frac{d}{d t}\left[\psi_{i}(t+2 \pi)-\psi_{i}(t)\right]=\sum_{j=1}^{n} \theta_{i j}\left[\psi_{j}(t+2 \pi)-\psi_{j}(t)\right],
$$

and consequently

$$
\text { (109) } \psi_{i}(t+2 \pi)-\psi_{i}(t)=\sum_{j=1}^{n-2} C_{j}^{\prime} e^{a_{i} t} \phi_{i j}(t)+\left[A^{\prime}+B^{\prime} t\right] e^{a_{n} t} \phi_{i n}(t)+B^{\prime} e^{a_{n} t} \phi_{i(n-1)}(t) \text {. }
$$

The terms included under the summation sign are obviously the same as those treated in Case I. Neglecting these we may write

$$
\psi_{i}(t)=e^{a_{n} t} \phi_{i n}(t) \cdot f_{i n}(t)+e^{a_{n} t} \cdot \phi_{i(n-1)}(t) f_{i(n-1)}(t)+\text { periodic terms, }
$$

where $f_{\text {in }}(t)$ and $f_{i(n-1)}(t)$ are functions whose forms are to be determined. Let us form the difference $\psi_{i}(t+2 \pi)-\psi_{i}(t)$ by means of $(110)$ and compare the result with (109). From (110) we get

$$
\begin{aligned}
\psi_{i}(t+2 \pi)-\psi_{i}(t)=\left[e^{2 a_{n} \pi}\right. & \left.f_{i n}(t+2 \pi)-f_{i n}(t)\right] e^{a_{n} t} \phi_{i n}(t) \\
& +\left[e^{2 a_{n} \pi} f_{i(n-1)}(t)-f_{i(n-1)}(t)\right] e^{a_{n} t} \phi_{i(n-1)}(t) .
\end{aligned}
$$


Comparing this with (109) we see that we must have

$$
\begin{aligned}
e^{2 a_{n} \pi} f_{i n}(t+2 \pi)-f_{i n}(t) & =A^{\prime}+B^{\prime} t \\
e^{2 a_{n} \pi} f_{i(n-1)}(t+2 \pi)-f_{i(n-1)}(t) & =B^{\prime} .
\end{aligned}
$$

Let us now define two new functions

$$
\begin{aligned}
\lambda_{i n}(t) & =e^{a_{n} t} f_{i n}(t)+\left[\frac{A^{\prime}}{1-e^{2 a_{n} \pi}}+\frac{2 \pi B^{\prime} e^{2 a_{n} \pi}}{\left(1-e^{2 a_{n} \pi}\right)^{2}}\right] e^{a_{n} t}+\frac{B^{\prime} t e^{a_{n} t}}{1-e^{2 a_{n} \pi}}, \\
\lambda_{i(n-1)}(t) & =e^{a_{n} t} f_{i(n-1)}(t)+\frac{B^{\prime} e^{a_{n} t}}{1-e^{2 a_{n} \pi}} .
\end{aligned}
$$

By virtue of the relations (112), $\lambda_{i n}(t)$ and $\lambda_{i(n-1)}(t)$ are periodic with the period $2 \pi$, and consequently

$$
\begin{aligned}
f_{i n}(t) & =e^{-a_{n} t} \lambda_{i n}(t)+\left[\begin{array}{c}
A^{\prime} \\
e^{2 a_{n} \pi}-1
\end{array}-\frac{2 \pi B^{\prime} e^{2 a_{n} \pi}}{\left(e^{2 a_{n} \pi}-1\right)^{2}}\right]+\frac{B^{\prime} t}{e^{2 a_{n} \pi}-1}, \\
f_{i(n-1)}(t) & =e^{-a_{n} t} \lambda_{i(n-1)}+B^{\prime} \\
e^{2 a_{n} \pi}-1 &
\end{aligned}
$$

where $\lambda_{i n}(t)$ and $\lambda_{i(n-1)}(t)$ are periodic with the period $2 \pi$. Substituting these expressions in $(110)$, we find

$$
\begin{aligned}
\psi_{i}(t)=\left[\frac{A^{\prime}}{\left(e^{2 a_{n} \pi}-1\right)}-\frac{2 \pi B^{\prime} e^{2 a_{n} \pi}}{\left(e^{2 a_{n} \pi}-1\right)^{2}}+\frac{B^{\prime} t}{\left(e^{2 a_{n} \pi}-1\right)}\right] e^{a_{n} t} \phi_{i n}(t) \\
\\
+\frac{B^{\prime}}{\left(e^{2 a_{n} \pi}-1\right)} e^{a_{n} t} \phi_{i(n-1)}(t)+\text { periodic terms. }
\end{aligned}
$$

Comparing these terms with (109) we see that they are merely terms of the complementary function and that therefore

$$
x_{i}=\text { complementary function }+ \text { periodic terms. }
$$

These results hold provided $\alpha_{n} \neq 0 \bmod V-\overline{1} . \quad$ In the event $\alpha_{n} \equiv 0 \bmod V-1$ equations' (112) become

$$
f_{i n}(t+2 \pi)-f_{i n}(t)=A^{\prime}+B^{\prime} t, \quad f_{i(n-1)}(t+2 \pi)-f_{i(n-1)}^{\prime}(t)=B^{\prime} .
$$

It is necessary to give new definitions to the $\lambda$ functions. We will let

$$
\lambda_{i n}(t)=f_{i n}(t)-\frac{B^{\prime}}{4 \pi} t^{2}-\frac{A^{\prime}-B^{\prime} \pi}{2 \pi} t, \quad \lambda_{i(n-1)}(t)=f_{i(n-1)}(t)-\frac{B^{\prime}}{2 \pi} t,
$$

which give

$$
f_{i n}(t)=\lambda_{i n}(t)+\frac{B^{\prime}}{4 \pi} t^{2}+\frac{A^{\prime}-B^{\prime} \pi}{2 \pi} t, \quad f_{i(n-1)}(t)=\lambda_{i(n-1)}(t)+\frac{B^{\prime}}{2 \pi} t .
$$


It is readily verified that $\lambda_{i n}(t)$ and $\lambda_{i(n-1)}(t)$ are periodic by virtue of (116). Substituting these expressions in (110), we find

$$
\Psi_{i}(t)=e^{a_{n} t}\left\{\left[\frac{B^{\prime}}{4 \pi} t^{2}+\frac{A^{\prime}-B^{\prime} \pi}{2 \pi} t\right] \phi_{i n}(t)+\frac{B^{\prime}}{2 \pi} t \phi_{i(n-1)}(t)\right\}+\text { periodic terms. }
$$

If we let

$$
\frac{A^{\prime}-B^{\prime} \pi}{2 \pi}=C, \quad \frac{B^{\prime}}{2 \pi}=D
$$

we get

$$
\psi_{i}(t)=e^{a_{n} t}\left\{\left[C t+\frac{1}{2} D t^{2}\right] \phi_{i n}(t)+D t \phi_{i(n-1)}(t)\right\}+\text { periodic terms. }
$$

This expression is not quite the same as $t$ times the corresponding complementary function for in one term we have the coefficient $\frac{1}{2} D$ instead of $D$. The theorem for this case then is

Theorem III. If $\theta_{i j}$ and $g_{i}(t)$ are periodic with the period $2 \pi$, and if two of the $\alpha_{j}$ are equal but not congruent to zero $\bmod \sqrt{-1}$, then the particular solution consists of terms periodic with the period $2 \pi$ plus a constant times the corresponding complementary function. But if two of the $\alpha_{j}$ are equal and congruent to zero so that the corresponding part of the complementary function has the form

$$
e^{a_{n} t}\left\{(A+B t) \phi_{i n}(t)+B \phi_{i(n-1)}(t)\right\}
$$

then the particular solution consists of a periodic function plus a term of the form

$$
e^{a_{n} t}\left\{\left(C t+\frac{1}{2} D t^{2}\right) \phi_{i n}(t)+D t \phi_{i(n-1)}(t)\right\} \text {. }
$$

§14. Special theorems for the equations of variation.

The foregoing theorems presuppose merely the conditions that the coefficients are periodic with the period $2 \pi$. Further facts with regard to the solutions may be established when further facts are specified with regard to the coefficients of the differential equations. Our equations of variation (51) may be written

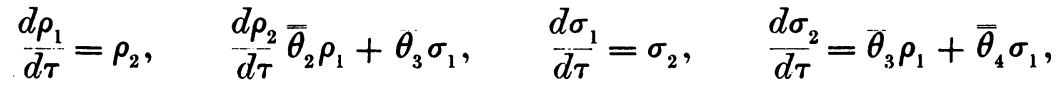

where the notation with respect to the $\theta$ 's has the following significance: even subscripts denote functions even in $\tau$, and odd subscripts denote functions odd in $\tau$; one dash indicates that only odd multiples of $\tau$ are involved, and two dashes indicate that only even multiples of $\tau$ are involved. The solutions, equations (82) and (83), may be characterized in the same manner, and are 
then

$$
\begin{aligned}
& \rho_{1}=A \bar{\alpha}_{2}(\tau)+B \bar{\alpha}_{1}(\tau)+C \bar{\alpha}_{3}(\tau)+D\left[\bar{\alpha}_{4}(\tau)+\tau \bar{\alpha}_{3}(\tau)\right], \\
& \rho_{2}=A \bar{\beta}_{1}(\tau)+B \bar{\beta}_{2}(\tau)+C \bar{\beta}_{4}(\tau)+D\left[\bar{\beta}_{3}(\tau)+\tau \bar{\beta}_{4}(\tau)\right], \\
& \sigma_{1}=A \bar{\gamma}_{1}(\tau)+B \bar{\gamma}_{2}(\tau)+C \bar{\gamma}_{4}(\tau)+D\left[\bar{\gamma}_{3}(\tau)+\tau \bar{\gamma}_{4}(\tau)\right], \\
& \sigma_{2}=A \bar{\delta}_{2}(\tau)+B \bar{\delta}_{1}(\tau)+C \bar{\delta}_{3}(\tau)+D\left[\bar{\delta}_{4}(\tau)+\tau \bar{\delta}_{3}(\tau)\right],
\end{aligned}
$$

where the notation is the same as for the $\theta$ 's with the exception that in the first two solutions every integral multiple of $\tau$ is increased by $\pm \lambda \tau$, e. g., $\cos (3+\lambda) \tau$. On these terms the dashes refer only to the integral part of the coefficients of $\tau$.

Suppose now we have the following non-homogeneous equations:

$$
\begin{array}{ll}
\frac{d \rho_{1}}{d \tau}=\rho_{2}, & \frac{d \rho_{2}}{d \tau}=\bar{\theta}_{2} \rho_{1}+\bar{\theta}_{3} \sigma_{1}+g(\tau), \\
\frac{d \sigma_{1}}{d \tau}=\sigma_{2}, & \frac{d \sigma_{2}}{d \tau}=\bar{\theta}_{3} \rho_{1}+\bar{\theta}_{4} \sigma_{1}+f(\tau),
\end{array}
$$

where $g(\tau)$ and $f(\tau)$ are periodic with the period $2 \pi$. Since the characteristic exponents are $\sqrt{-1} \lambda,-\sqrt{-1} \lambda, 0,0$, by Theorem III the solution has the form

$$
\begin{aligned}
& \rho_{1}=\left(\rho_{1}\right)+\xi_{1}=\left(\rho_{1}\right)+\omega_{1}(\tau)+a \tau \bar{\alpha}_{3}+b\left[\frac{1}{2} \tau^{2} \bar{\alpha}_{3}+\tau \bar{\alpha}_{4}\right], \\
& \rho_{2}=\left(\rho_{2}\right)+\xi_{2}=\left(\rho_{2}\right)+\omega_{2}(\tau)+a \tau \bar{\beta}_{4}+b\left[\frac{1}{2} \tau^{2} \bar{\beta}_{4}+\tau \bar{\beta}_{3}\right], \\
& \sigma_{1}=\left(\sigma_{1}\right)+\eta_{1}=\left(\sigma_{1}\right)+\omega_{3}(\tau)+a \tau \bar{\gamma}_{4}+b\left[\frac{1}{2} \tau^{2} \bar{\gamma}_{4}+\tau \bar{\gamma}_{3}\right], \\
& \sigma_{2}=\left(\sigma_{1}\right)+\eta_{2}=\left(\sigma_{2}\right)+\omega_{4}(\tau)+a \tau \bar{\delta}_{3}+b\left[\frac{1}{2} \tau^{2} \bar{\delta}_{3}+\tau \bar{\delta}_{4}\right],
\end{aligned}
$$

where $\left(\rho_{i}\right),\left(\sigma_{i}\right)$ indicate the complementary function and $\xi_{i}$ and $\eta_{i}$ are the particular integrals, of which the $\omega_{i}$ are the periodic parts. The $a$ and $b$ are constants which depend upon the differential equations. Since the $\xi_{i}$ and $\eta_{i}$ satisfy the differential equations we have

$$
\begin{array}{cc}
\frac{d}{d \tau} \xi_{1}(\tau)=\xi_{2}(\tau), & \frac{d}{d \tau} \xi_{2}(\tau)=\bar{\theta}_{2} \xi_{1}(\tau)+\bar{\theta}_{3} \eta_{1}(\tau)+g(\tau), \\
\frac{d}{d \tau} \eta_{1}(\tau)=\eta_{2}(\tau), & \frac{d}{d \tau} \eta_{2}(\tau)=\bar{\theta}_{3} \xi_{1}(\tau)+\bar{\theta}_{4} \eta_{1}(\tau)+f(\tau) .
\end{array}
$$

Changing $\tau$ into $-\tau$ in these equations, we get

$$
\begin{array}{ll}
\frac{d}{d \tau} \xi_{1}(-\tau)=-\xi_{2}(-\tau), & \frac{d}{d \tau} \xi_{2}(-\tau)=-\bar{\theta}_{2} \xi_{1}(-\tau)+\bar{\theta}_{3} \eta_{1}(-\tau)-g(-\tau), \\
\frac{d}{d \tau} \eta_{1}(-\tau)=-\eta_{2}(-\tau), & \frac{d}{d \tau} \eta_{2}(-\tau)=+\theta_{3} \xi_{1}(-\tau)-\bar{\theta}_{4} \eta_{1}(-\tau)-f(-\tau) .
\end{array}
$$


If now we make the additional hypothesis that $g(\tau)$ is an even function of $\tau$ and $f(\tau)$ is an odd function, equations (124) and (125) may be combined into the following set

$$
\begin{aligned}
& \frac{d}{d \tau}\left[\xi_{1}(\tau)-\xi_{1}(-\tau)\right]=\left[\xi_{2}(\tau)+\xi_{2}(-\tau)\right], \\
& \frac{d}{d \tau}\left[\xi_{2}(\tau)+\xi_{2}(-\tau)\right]=\bar{\theta}_{2}\left[\xi_{1}(\tau)-\xi_{1}(-\tau)\right]+\bar{\theta}_{3}\left[\eta_{1}(\tau)+\eta_{1}(-\tau)\right] \\
& \frac{d}{d \tau}\left[\eta_{1}(\tau)+\eta_{1}(-\tau)\right]=\left[\eta_{2}(\tau)-\eta_{2}(-\tau)\right], \\
& \frac{d}{d \tau}\left[\eta_{2}(\tau)-\eta_{2}(-\tau)\right]=\theta_{3}\left[\xi_{1}(\tau)-\xi_{1}(-\tau)\right]+\bar{\theta}_{4}\left[\eta_{1}(\tau)+\eta_{1}(-\tau)\right] .
\end{aligned}
$$

These equations are the same as (120). Therefore

$$
\begin{aligned}
& \xi_{1}(\tau)-\xi_{1}(-\tau)=A \bar{\alpha}_{2}(\tau)+B \bar{\alpha}_{1}(\tau)+C \bar{\alpha}_{3}(\tau)+D\left[\bar{\alpha}_{4}(\tau)+\tau \bar{\alpha}_{3}(\tau)\right] \\
& \xi_{2}(\tau)+\xi_{2}(-\tau)=A \beta_{1}(\tau)+B \bar{\beta}_{2}(\tau)+C \bar{\beta}_{4}(\tau)+D\left[\bar{\beta}_{3}(\tau)+\tau \bar{\beta}_{4}(\tau)\right] \\
& \eta_{1}(\tau)+\eta_{1}(-\tau)=A \bar{\gamma}_{1}(\tau)+B \bar{\gamma}_{2}(\tau)+C \bar{\gamma}_{4}(\tau)+D\left[\bar{\gamma}_{3}(\tau)+\tau \bar{\gamma}_{4}(\tau)\right] \\
& \eta_{2}(\tau)-\eta_{2}(-\tau)=A \bar{\delta}_{2}(\tau)+B \bar{\delta}_{1}(\tau)+C \delta_{3}(\tau)+D\left[\delta_{4}(\tau)+\tau \bar{\delta}_{3}(\tau)\right]
\end{aligned}
$$

Putting $\tau=0$ in these equations we get from the first and the fourth

$$
0=A \alpha_{2}(0)+D \alpha_{4}(0), \quad 0=A \delta_{2}(0)+D \delta_{4}(0) .
$$

Either $A=D=0$, or the determinant $\alpha_{2}(0) \delta_{4}(0)-\delta_{2}(0) \alpha_{4}(0)=0$. But it is readily verified that the determinant is not zero. Therefore $A=D=0$. If we suppose that $\xi_{2}(0)=\eta_{1}(0)=0$ (we shall be interested only in such cases), it follows from the second and third equations of (127) that

$$
0=B \bar{\beta}_{2}(0)+C \bar{\beta}_{4}(0), \quad 0=B \bar{\gamma}_{2}(0)+C \bar{\gamma}_{4}(0),
$$

and hence $B=C=0$. Consequently

$$
\begin{array}{rr}
\xi_{1}(\tau)-\xi_{1}(-\tau)=0, & { }_{2}(\tau)+\xi_{2}(-\tau)=0, \\
\eta_{1}(\tau)+\eta_{1}(-\tau)=0, & \eta_{2}(\tau)-\eta_{2}(-\tau)=0 .
\end{array}
$$

We have then

TheOREM IV. If $g(\tau)$ is an even function of $\tau$ and $f(\tau)$ is an odd function of $\tau$, and if $\xi_{2}(0)=\eta_{1}(0)=0$, then $\xi_{1}(\tau)$ and $\eta_{2}(\tau)$ are even functions of $\tau$, and $\xi_{2}(\tau)$ and $\eta_{1}(\tau)$ are odd functions of $\tau$. 
In the same way it can be shown that if $g(\tau)$ is odd and $f(\tau)$ is even, and if $\xi_{1}(0)=\eta_{2}(0)=0$, then $\xi_{1}$ and $\eta_{2}$ are odd and $\xi_{2}$ and $\eta_{1}$ are even.

Let us suppose now that $g(\tau)$ is periodic and contains only even multiples of $\tau$, and that $f(\tau)$ is periodic and contains only odd multiples of $\tau$. The general form of the solution will be the same as (123). $\xi_{1}, \xi_{2}, \eta_{1}$, and $\eta_{2}$ satisfy the differential equations

$$
\begin{aligned}
\frac{d}{d \tau} \xi_{1}(\tau) & =\xi_{2}(\tau), & \frac{d}{d \tau} \xi_{2}(\tau) & =\bar{\theta}_{2} \xi_{1}(\tau)+\bar{\theta}_{3} \eta_{1}(\tau)+\bar{g}(\tau), \\
\frac{d}{d \tau} \eta_{1}(\tau) & =\eta_{2}(\tau), & \frac{d}{d \tau} \eta_{2}(\tau) & =\bar{\theta}_{3} \xi_{1}(\tau)+\bar{\theta}_{4} \eta_{1}(\tau)+\bar{f}(\tau) .
\end{aligned}
$$

Let us denote $\xi_{i}(\tau+\pi)$ by $\xi_{i}^{\prime}(\tau)$ and $\eta_{i}(\tau+\pi)$ by $\eta_{i}^{\prime}(\tau)$. Then by changing $\tau$ into $\tau+\pi$ in (130) we bave

$$
\begin{array}{rlrl}
\frac{d}{d \tau} \xi_{1}^{\prime}(\tau) & =\xi_{2}^{\prime}(\tau), & \frac{d}{d \tau} \xi_{2}^{\prime}(\tau) & =\bar{\theta}_{2} \xi_{1}^{\prime}(\tau)-\theta_{3} \eta_{1}^{\prime}(\tau)+\bar{g}(\tau), \\
\frac{d}{d \tau} \eta_{1}^{\prime}(\tau)=\eta_{2}^{\prime}(\tau), & \frac{d}{d \tau} \eta_{2}^{\prime}(\tau)=-\bar{\theta}_{3} \xi_{1}^{\prime}(\tau)+\bar{\theta}_{4} \eta_{1}^{\prime}(\tau)-\bar{f}(\tau) .
\end{array}
$$

From (130) and (131) it follows that

$$
\begin{array}{lc}
\frac{d}{d \tau}\left[\xi_{1}-\xi_{1}^{\prime}\right]=\left[\xi_{2}-\xi_{2}^{\prime}\right], & \frac{d}{d \tau}\left[\xi_{2}-\xi_{2}^{\prime}\right]=\bar{\theta}_{2}\left[\xi_{1}-\xi_{1}^{\prime}\right]+\theta_{3}\left[\eta_{1}+\eta_{1}^{\prime}\right], \\
\frac{d}{d \tau}\left[\eta_{1}+\eta_{1}^{\prime}\right]=\left[\eta_{2}+\eta_{2}^{\prime}\right], & \frac{d}{d \tau}\left[\eta_{2}+\eta_{2}^{\prime}\right]=\bar{\theta}_{3}\left[\xi_{1}-\xi_{1}^{\prime}\right]+\bar{\theta}_{4}\left[\eta_{1}+\eta_{1}^{\prime}\right] .
\end{array}
$$

The solutions of these equations, which have the form of $(120)$, are

$$
\begin{aligned}
& \xi_{1}-\xi_{1}^{\prime}=A \bar{\alpha}_{2}(\tau)+B \alpha_{1}(\tau)+C \bar{\alpha}_{3}(\tau)+D\left[\bar{\alpha}_{4}(\tau)+\tau \bar{\alpha}_{3}(\tau)\right], \\
& \xi_{2}-\xi_{2}^{\prime}=A \bar{\beta}_{1}(\tau)+B \bar{\beta}_{2}(\tau)+C \bar{\beta}_{4}(\tau)+D\left[\bar{\beta}_{3}(\tau)+\tau \bar{\beta}_{4}(\tau)\right], \\
& \eta_{1}+\eta_{1}^{\prime}=A \bar{\gamma}_{1}(\tau)+B \bar{\gamma}_{2}(\tau)+C \bar{\gamma}_{4}(\tau)+D\left[\gamma_{3}(\tau)+\tau \bar{\gamma}_{4}(\tau)\right], \\
& \eta_{2}+\eta_{2}^{\prime}=A \bar{\delta}_{2}(\tau)+B \bar{\delta}_{1}(\tau)+C \delta_{3}(\tau)+D\left[\delta_{4}(\tau)+\tau \delta_{3}(\tau)\right] .
\end{aligned}
$$

Forming these expressions directly from (123), we get

$$
\begin{aligned}
& \xi_{1}-\xi_{1}^{\prime}=\omega_{1}(\tau)-\omega_{1}(\tau+\pi)-\left[a \pi+\frac{1}{2} b \pi^{2}\right] \bar{\alpha}_{3}(\tau)-b \pi\left[\tau \bar{\alpha}_{3}(\tau)+\bar{\alpha}_{4}(\tau)\right], \\
& \xi_{2}-\xi_{2}^{\prime}=\omega_{2}(\tau)-\omega_{2}(\tau+\pi)-\left[a \pi+\frac{1}{2} b \pi^{2}\right] \bar{\beta}_{4}(\tau)-b \pi\left[\tau \bar{\beta}_{4}(\tau)+\bar{\beta}_{3}(\tau)\right], \\
& \eta_{1}+\eta_{1}^{\prime}=\omega_{3}(\tau)+\omega_{3}(\tau+\pi)-\left[a \pi+\frac{1}{2} b \pi^{2}\right] \bar{\gamma}_{4}(\tau)-b \pi\left[\tau \gamma_{4}(\tau)+\bar{\gamma}_{3}(\tau)\right], \\
& \eta_{2}+\eta_{2}^{\prime}=\omega_{4}(\tau)+\omega_{4}(\tau+\pi)-\left[a \pi+\frac{1}{2} b \pi^{2}\right] \delta_{3}(\tau)-b \pi\left[\tau \delta_{3}(\tau)+\delta_{4}(\tau)\right] .
\end{aligned}
$$


Comparing (133) and (134) we see that

$$
\begin{aligned}
& A=B=0, \quad C=-\left[a \pi+\frac{1}{2} b \pi^{2}\right], \quad D=-b \pi, \\
& \omega_{1}(\tau)-\omega_{1}(\tau+\pi)=0, \quad \omega_{3}(\tau)+\omega_{3}(\tau+\pi)=0, \\
& \omega_{2}(\tau)-\omega_{2}(\tau+\pi)=0, \quad \omega_{4}(\tau)+\omega_{4}(\tau+\pi)=0 .
\end{aligned}
$$

Therefore $\omega_{1}(\tau)$ and $\omega_{2}(\tau)$ contain only even multiples of $\tau$ while $\omega_{3}(\tau)$ and $\omega_{4}(\tau)$ contain only odd multiples, and by carrying this result into (123) we find

$$
\begin{aligned}
& \xi_{1}=\bar{\omega}_{1}(\tau)+a \tau \bar{\alpha}_{3}(\tau)+b\left[\frac{1}{2} \tau^{2} \bar{\alpha}_{3}(\tau)+\tau \bar{\alpha}_{4}(\tau)\right], \\
& \xi_{2}=\bar{\omega}_{2}(\tau)+a \tau \bar{\beta}_{4}(\tau)+b\left[\frac{1}{2} \tau^{2} \bar{\beta}_{4}(\tau)+\tau \bar{\beta}_{3}(\tau)\right], \\
& \eta_{1}=\bar{\omega}_{3}(\tau)+a \tau \bar{\gamma}_{4}(\tau)+b\left[\frac{1}{2} \tau^{2} \bar{\gamma}_{4}(\tau)+\tau \bar{\gamma}_{3}(\tau)\right], \\
& \eta_{2}=\bar{\omega}_{4}(\tau)+a \tau \bar{\delta}_{3}(\tau)+b\left[\frac{1}{2} \tau^{2} \bar{\delta}_{3}(\tau)+\tau \bar{\delta}_{4}(\tau)\right],
\end{aligned}
$$

and hence we have

Theоrem V. If $g(\tau)$ contains only even multiples of $\tau$ and $f(\tau)$ contains only odd multiples, then $\xi_{1}$ and $\xi_{2}$ contain only even multiples of $\tau$ and $\eta_{1}$ and $\eta_{2}$ contain only odd multiples.

If in addition to the above hypotheses we suppose that $g(\tau)$ is an even function of $\tau$ and $f(\tau)$ is an odd function, then $\xi_{1}$ and $\eta_{2}$ are even functions of $\tau$ and $\xi_{2}$ and $\eta_{1}$ are odd functions. Therefore $b=0$. But if $g(\tau)$ is an odd function and $f(\tau)$ is an even function, then $\xi_{1}$ and $\eta_{2}$ are odd functions and $\xi_{2}$ and $\eta_{1}$ are even functions, so that in this event $a=0$.

In the same manner as above we prove

Theorem VI. If $g(\tau)$ contains only odd multiples of $\tau$ and $f(\tau)$ contains only even multiples, then $\xi_{1}$ and $\xi_{2}$ contain only odd multiples of $\tau$ and $\eta_{1}$ and $\eta_{2}$ contain only even multiples. Furthermore $\xi_{1}, \xi_{2}, \eta_{1}$ and $\eta_{2}$ are periodic with the period $2 \pi$.

If $g(\tau)$ is of the form $\sum_{j} m_{j} \cos (j \pm \lambda) \tau$ and $f(\tau)$ has the form $\sum_{j} n_{j} \sin (j \pm \lambda) \tau$ then, since $\pm \sqrt{-1} \lambda$ are the characteristic exponents of the homogeneous equations, the form of the solution is, by Theorem II,

$$
\begin{aligned}
& \xi_{1}=\sum_{k} p_{k}^{(1)} \cos (k \pm \lambda) \tau+\sum_{k} p_{k}^{(2)} \sin (k \pm \lambda) \tau+a^{(1)} \tau \bar{\alpha}_{1}(\tau)+a^{(2)} \tau \bar{\alpha}_{2}(\tau) \\
& \xi_{2}=\sum_{k} q_{k}^{(2)} \cos (k \pm \lambda) \tau+\sum_{k} q_{k}^{(1)} \sin (k \pm \lambda) \tau+a^{(1)} \tau \bar{\beta}_{2}(\tau)+a^{(2)} \tau \bar{\beta}_{1}(\tau), \\
& \eta_{1}=\sum_{k} r_{k}^{(2)} \cos (k \pm \lambda) \tau+\sum_{k} r_{k}^{(1)} \sin (k \pm \lambda) \tau+a^{(1)} \tau \bar{\gamma}_{2}(\tau)+a^{(2)} \tau \bar{\gamma}_{1}(\tau) \\
& \eta_{2}=\sum_{k} s_{k}^{(1)} \cos (k \pm \lambda) \tau+\sum_{k} s_{k}^{(2)} \sin (k \pm \lambda) \tau+a^{(1)} \tau \bar{\delta}_{1}(\tau)+a^{(2)} \tau \bar{\delta}_{2}(\tau)
\end{aligned}
$$

but since $g(\tau)$ is an even function and $f(\tau)$ is an odd function, $\xi_{1}$ and $\eta_{2}$ are 
even functions and $\xi_{2}$ and $\eta_{1}$ are odd functions. Therefore all the coefficients in (136) which have the upper index (2) are zero. But if $g(\tau)$ were an odd function of $\tau$ and $f(\tau)$ an even function then all the coefficients in (136) which have the upper index (1) would be zero. Therefore

Theorem VII. If $g(\tau)$ is of the form $\sum_{j} m_{j} \cos (j \pm \lambda) \tau$ and $f(\tau)$ has the form $\sum_{j} n_{j} \sin (j \pm \lambda) \tau$, where $\pm \sqrt{-1} \lambda$ are the characteristic exponents of the homogeneous equations, then the particular solution has the form

$$
\begin{aligned}
& \xi_{1}=\sum_{a} p_{a} \cos (a \pm \lambda) \tau+A \tau \bar{\alpha}_{1}(\tau), \\
& \xi_{2}=\sum_{b} p_{b} \sin (b \pm \lambda) \tau+A \tau \bar{\beta}_{2}(\tau), \\
& \eta_{1}=\sum_{c} p_{c} \sin (c \pm \lambda) \tau+A \tau \overline{\bar{\gamma}}_{2}(\tau), \\
& \eta_{2}=\sum_{d} p_{d} \cos (d \pm \lambda) \tau+A \tau \bar{\delta}_{1}(\tau) .
\end{aligned}
$$

Also,

Theorem VIII. If $g(\tau)$ has the form $\sum_{j} m_{j} \sin (j \pm \lambda) \tau$ and $f(\tau)$ has the form $\sum_{j} n_{j} \cos (j \pm \lambda) \tau$, where $\pm \sqrt{-1} \lambda$ are the characteristic exponents of the homogeneous equations, then the particular solution has the form

$$
\begin{array}{ll}
\xi_{1}=\sum_{a} r_{a} \sin (a \pm \lambda) \tau+B \tau \bar{\alpha}_{2}(\tau), & \xi_{2}=\sum_{b} r_{b} \cos (b \pm \lambda) \tau+B \tau \bar{\beta}_{1}(\tau), \\
\eta_{1}=\sum_{c} r_{c} \cos (c \pm \lambda) \tau+B \tau \bar{\gamma}_{1}(\tau), & \eta_{2}=\sum_{d} r_{d} \sin (d \pm \lambda) \tau+B \tau \bar{\delta}_{2}(\tau) .
\end{array}
$$

It is understood that in the above two theorems $a, b, c, d$, and $j$ are integers.

\section{\$15. Integration of the complete differential equations (50).}

It will be convenient hereafter to use the following notation for the solutions of the equations of variation:

$$
\begin{aligned}
& \rho=A \alpha_{2}(\tau)+B \alpha_{1}(\tau)+C \alpha_{3}(\tau)+D\left[\alpha_{4}(\tau)+\tau \alpha_{3}(\tau)\right], \\
& \sigma=A \gamma_{1}(\tau)+B \gamma_{2}(\tau)+C \gamma_{4}(\tau)+D\left[\gamma_{3}(\tau)+\tau \gamma_{4}(\tau)\right] .
\end{aligned}
$$

The $\alpha_{j}(\tau)$ and $\gamma_{j}(\tau)$ are characterized thus :

$$
\begin{aligned}
& \alpha_{2}(\tau) \text { involves only terms of the form } \cos [(2 n+1) \pm \lambda] \tau,
\end{aligned}
$$

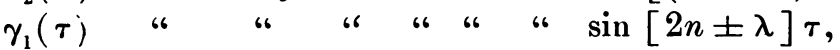

$$
\begin{aligned}
& \alpha_{1}(\tau) \quad \text { " } \quad \text { " } \quad \text { " } \quad \text { " } \quad \text { " } \sin [(2 n+1) \pm \lambda] \tau \text {, }
\end{aligned}
$$

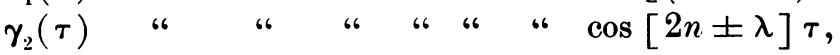

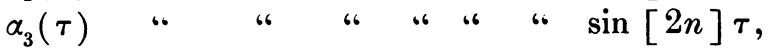

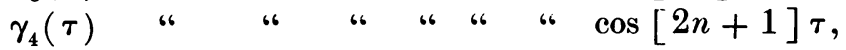

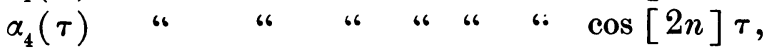

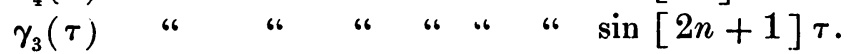


It will be convenient also to write the differential equations for $\rho$ and $\sigma$ as

$$
\begin{array}{lr}
\rho^{\prime \prime}+\theta_{2} \rho+\theta_{3} \sigma=\theta_{001} \epsilon+\theta_{101} \epsilon \rho+\theta_{200} \rho^{2}+\theta_{110} \rho \sigma+\theta_{020} \sigma^{2}+\cdots, \\
\sigma^{\prime \prime}+\theta_{4} \sigma+\theta_{3} \rho= & \theta_{210} \rho^{2}+\theta_{110} \rho \sigma+\theta_{020} \sigma^{2}+\cdots,
\end{array}
$$

where all the $\theta$ 's are periodic with the period $2 \pi$, and $\theta_{2}$ and $\theta_{4}$ contain only cosines of even multiples of $\tau$, and $\theta_{3}$ contains only sines of odd multiples of $\tau$. On the right side of the first equation the coefficients of terms carrying odd powers of $\sigma$ contain only sines of odd multiples of $\tau$. All the other coefficients contain only cosines of even multiples. In the second equation odd powers of $\sigma$ have coefficients involving only cosines of even multiples. All other coeffcients contain only sines of odd multiples.

The initial conditions are

$$
\rho=\alpha, \quad \sigma=0, \quad \rho^{\prime}=0, \quad \sigma^{\prime}=\delta .
$$

$\mathrm{W}$ e will integrate equations (138) as power series in $\alpha, \delta$ and $\epsilon, \tau$ entering into the coefficients. From Poincaré's extension of Cauchy's theorem we know that these series are convergent for any arbitrarily chosen interval for $\tau, 0 \leqq \tau \leqq T$, provided $|\alpha|,|\delta|$ and $|\epsilon|$ are sufficiently small. The equations of variation involve the period $2 \pi / \lambda$. The solutions are not periodic unless $\lambda$ is rational. Hence $\theta_{1}^{2}$ nust be chosen in advance so that $\lambda$ is rational. We will suppose then that $\lambda=J / K$, where $J$ and $K$ are integers, relatively prime. Then three solutions of the equations of variation are periodic with the period $2 K \pi$.

Since $\rho$ and $\sigma$ are expansible in powers of $\alpha, \delta$ and $\epsilon$, we may write

$$
\begin{aligned}
& \rho=\rho_{100} \alpha+\rho_{010} \delta+\rho_{001} \epsilon+\rho_{200} \alpha^{2}+\rho_{110} \alpha \delta+\rho_{020} \delta^{2}+\rho_{101} \alpha \epsilon+\rho_{011} \delta \epsilon+\rho_{002} \epsilon^{2}+\cdots, \\
& \sigma=\sigma_{100} \alpha+\sigma_{010} \delta+\sigma_{001} \epsilon+\sigma_{200} \alpha^{2}+\sigma_{110} \alpha \delta+\sigma_{020} \delta^{2}+\sigma_{101} \alpha \epsilon+\sigma_{011} \rho \epsilon+\sigma_{002} \epsilon^{2}+\cdots
\end{aligned}
$$

Substituting these expressions in the differential equations (138) and equating the coefficients of similar powers, we have :

Coefficient of $\alpha$.

$$
\begin{aligned}
& \rho_{100}^{\prime \prime}+\theta_{2} \rho_{100}+\theta_{3} \sigma_{100}=0, \\
& \sigma_{100}^{\prime \prime}+\theta_{4} \sigma_{100}+\theta_{3} \rho_{100}=0 .
\end{aligned}
$$

The solution of these equations is

$$
\begin{aligned}
& \rho_{100}=A \alpha_{2}(\tau)+B \alpha_{1}(\tau)+C \alpha_{3}(\tau)+D\left[\alpha_{4}(\tau)+\tau \alpha_{3}(\tau)\right], \\
& \sigma_{100}=A \gamma_{1}(\tau)+B \gamma_{2}(\tau)+C \gamma_{4}(\tau)+D\left[\gamma_{3}(\tau)+\tau \gamma_{4}(\tau)\right] .
\end{aligned}
$$

In order to satisfy the initial conditions we must have, at $\tau=0$,

$$
\rho_{100}=1, \quad \sigma_{100}=0, \quad \rho_{100}^{\prime}=0, \quad \sigma_{100}^{\prime}=0 .
$$


From these conditions we find

$$
\begin{aligned}
& A \alpha_{2}(0)+D \alpha_{4}(0)=1, \\
& B \alpha_{1}^{\prime}(0)+C \alpha_{3}^{\prime}(0)=0, \\
& B \gamma_{2}(0)+C \gamma_{4}(0)=0, \\
& A \gamma_{1}^{\prime}(0)+D\left[\gamma_{3}^{\prime}(0)+\gamma_{4}(0)\right]=0 .
\end{aligned}
$$

The solution of these conditional equations is

$$
\begin{aligned}
& B=0, \quad A=\frac{\gamma_{3}^{\prime}(0)+\gamma_{4}(0)}{\Delta}=A_{100}^{(1)}, \\
& C=0, \quad D=-\frac{\gamma_{1}^{\prime}(0)}{\Delta}=A_{100}^{(2)}, \\
& \Delta=\alpha_{2}(0)\left[\gamma_{3}^{\prime}(0)+\gamma_{4}(0)\right]-\alpha_{4}(0) \gamma_{1}^{\prime}(0) .
\end{aligned}
$$

Hence the solution is

$$
\begin{aligned}
& \rho_{100}=A_{100}^{(1)} \alpha_{2}(\tau)+A_{100}^{(2)}\left[\alpha_{4}(\tau)+\tau \alpha_{3}(\tau)\right], \\
& \sigma_{100}=A_{100}^{(1)} \gamma_{1}(\tau)+A_{100}^{(2)}\left[\gamma_{3}(\tau)+\tau \alpha_{4}(\tau)\right] .
\end{aligned}
$$

Coefficient of $\delta$.

$$
\begin{aligned}
& \rho_{010}^{\prime \prime}+\theta_{2} \rho_{010}+\theta_{3} \sigma_{010}=0, \\
& \sigma_{010}^{\prime \prime}+\theta_{4} \sigma_{010}+\theta_{3} \rho_{010}=0 .
\end{aligned}
$$

These equations are the same as for the coefficient of $\alpha$. From the initial conditions we must have at $\tau=0$

$$
\rho_{010}=0, \quad \sigma_{010}=0, \quad \rho_{010}^{\prime}=0, \quad \sigma_{010}^{\prime}=1 .
$$

The solutions are

where

$$
\begin{aligned}
& \rho_{010}=A_{010}^{(1)} \alpha_{2}(\tau)+A_{010}^{(2)}\left[\alpha_{4}(\tau)+\tau \alpha_{3}(\tau)\right], \\
& \sigma_{010}=A_{010}^{(1)} \gamma_{1}(\tau)+A_{010}^{(2)}\left[\gamma_{3}(\tau)+\tau \gamma_{4}(\tau)\right],
\end{aligned}
$$

$$
A_{010}^{(1)}=-\frac{\alpha_{4}(0)}{\Delta}, \quad A_{010}^{(2)}=\frac{\alpha_{2}(0)}{\Delta} \text {. }
$$

Coefficient of $\epsilon$.

$$
\begin{aligned}
& \rho_{001}^{\prime \prime}+\theta_{2} \rho_{001}+\theta_{3} \sigma_{001}=\theta_{001}, \\
& \sigma_{001}^{\prime \prime}+\theta_{4} \sigma_{001}+\theta_{3} \rho_{001}=0 .
\end{aligned}
$$

The right member, $\theta_{001}$, is a periodic function of $\tau$ with the period $2 \pi$. Furthermore it involves only cosines of even multiples of $\tau$. Hence by Theorem $V$ the 
solution has the form

$$
\begin{aligned}
& \rho_{001}=A \alpha_{2}(\tau)+B \alpha_{1}(\tau)+C \alpha_{3}(\tau)+D\left[\alpha_{4}(\tau)+\tau \alpha_{3}(\tau)\right]+\alpha_{5}(\tau)+a \tau \alpha_{3}(\tau), \\
& \sigma_{001}=A \gamma_{1}(\tau)+B \gamma_{2}(\tau)+C \gamma_{4}(\tau)+D\left[\gamma_{3}(\tau)+\tau \gamma_{4}(\tau)\right]+\gamma_{5}(\tau)+a \tau \gamma_{4}(\tau),
\end{aligned}
$$

where $a$ is a constant depending on $\theta_{001}, \alpha_{5}(\tau)$ is a cosine series involving only even multiples of $\tau$, and $\gamma_{5}(\tau)$ involves only sines of odd multiples of $\tau$.

From the initial conditions we must have at $\tau=0$

$$
\rho_{001}=0, \quad \sigma_{001}=0, \quad \rho_{001}^{\prime}=0, \quad \sigma_{001}^{\prime}=0 .
$$

Determining the constants of integration so as to satisfy these conditions we have the solution

where

$$
\begin{aligned}
& \rho_{001}=A_{001}^{(1)} \alpha_{2}(\tau)+A_{001}^{(2)}\left[\alpha_{4}(\tau)+\tau \alpha_{3}(\tau)\right]+\alpha_{6}(\tau), \\
& \sigma_{001}=A_{001}^{(1)} \gamma_{1}(\tau)+A_{001}^{(2)}\left[\gamma_{3}(\tau)+\tau \gamma_{4}(\tau)\right]+\gamma_{6}(\tau),
\end{aligned}
$$

$$
\begin{gathered}
A_{001}^{(1)}=\frac{1}{\Delta}\left[\alpha_{4} \beta_{6}^{\prime}-\alpha_{6}\left(\beta_{3}+\beta_{4}^{\prime}\right)\right], \\
A_{001}^{(2)}=\frac{1}{\Delta}\left[\alpha_{6} \beta_{1}^{\prime}-\alpha_{1} \beta_{6}^{\prime}\right], \\
\alpha_{6}(\tau)=\alpha_{5}(\tau)-a \alpha_{4}(\tau), \quad \gamma_{6}(\tau)=\gamma_{5}(\tau)-a \gamma_{3}(\tau) .
\end{gathered}
$$

It will be seen later that the value of $\alpha_{6}(\tau)$ for $\tau=0$ plays an important rôle, so that it is necessary for us to verify that it does not vanish. By hypothesis $\alpha_{5}(\tau)$ is the periodic part of the particular solution for $\rho$ in the differential equations (144). Let us put in these equations

$$
\rho_{001}=\phi+a \tau \alpha_{3}(\tau), \quad \sigma_{001}=\psi+a \tau \gamma_{4}(\tau),
$$

where $\phi$ and $\psi$ are the periodic parts of the particular solution. We find

$\phi^{\prime \prime}+\theta_{2} \phi+\theta_{3} \psi=-2 a \alpha_{3}^{\prime}(\tau)+1+\left[\left(-3 \theta_{1}^{2}+\frac{8}{4} \beta^{2}\right)-\frac{3}{4} \beta^{2} \cos 2 \tau\right] \mu^{2}+\cdots$, $\psi^{\prime \prime}+\theta_{1} \psi+\theta_{3} \phi=-2 a \beta_{3}^{\prime}(\tau)$

or,

$$
\begin{aligned}
& \phi^{\prime \prime}+\theta_{2} \phi+\theta_{3} \psi=1+\left[\left(-3 \theta_{1}^{2}+\frac{3}{4} \beta^{2}\right)-2\left(a_{0}+\frac{3}{4}\right) \beta^{2} \cos 2 \tau\right] \mu^{2}, \\
& \psi^{\prime \prime}+\theta_{4} \psi+\theta_{3} \psi=-2 a_{0} \beta \sin \tau \mu+\cdots,
\end{aligned}
$$

where we have substituted

$$
a=a_{0}+a_{2} \mu^{2}+\cdots
$$


The functions $\phi$ and $\psi$ can be expanded as series of the form

$$
\phi=\phi_{0}+\phi_{2} \mu^{2}+\cdots, \quad \psi=\psi_{1} \mu+\psi_{3} \mu^{3}+\cdots
$$

Then we get

$$
\begin{aligned}
& \phi_{0}^{\prime \prime}+\phi_{0}=1, \quad \text { so that } \quad \phi_{0}=1+c_{0} \cos \tau, \\
& \psi_{1}^{\prime \prime}+\psi_{1}=\left(3-2 a_{0}\right) \beta \sin \tau+\frac{3}{2} \beta c_{0} \sin 2 \tau .
\end{aligned}
$$

Since $\psi_{1}$ is periodic we must have $a_{0}=\frac{3}{2}$, and then

$$
\begin{gathered}
\psi_{1}=c_{1} \sin \tau-\frac{1}{2} \beta c_{0} \sin 2 \tau, \\
\phi_{2}^{\prime \prime}+\phi_{2}=-\frac{1}{4} c_{0} \beta^{2} \cos \tau+\text { other terms. }
\end{gathered}
$$

Since $\phi_{2}$ is periodic we must have $c_{0}=0$. Therefore

Consequently

$$
\begin{gathered}
\phi=\alpha_{5}(\tau)=1+\mu^{2} P_{1}\left(\mu^{2}\right), \quad a=\frac{3}{2}+\mu^{2} P_{2}\left(\mu^{2}\right), \\
\alpha_{4}(\tau)=0+\left[\frac{3}{2} \beta^{2}+\frac{1}{2} \beta^{2} \cos 2 \tau\right] \mu^{2}+\cdots
\end{gathered}
$$

$$
\alpha_{6}(0)=\alpha_{5}(0)-a \alpha_{4}(0)=1+\mu^{2} P_{3}\left(\mu^{2}\right),
$$

an expression which does not vanish identically.

Coefficient of $\alpha^{2}$.

$$
\begin{aligned}
& \rho_{200}^{\prime \prime}+\theta_{2} \rho_{200}+\theta_{3} \sigma_{200}=R_{200}, \\
& \sigma_{200}^{\prime \prime}+\theta_{4} \sigma_{200}+\theta_{3} \rho_{200}=S_{200} .
\end{aligned}
$$

The right members of these equations have the following expressions :

$$
\begin{aligned}
R_{200}= & A_{100}^{(1)}\left[\theta_{200} \alpha_{2}^{2}+\theta_{110} \alpha_{2} \gamma_{1}+\theta_{020} \gamma_{1}^{2}\right] \\
& +A_{100}^{(1)} A_{100}^{(2)}\left[2 \theta_{200} \alpha_{2}\left(\tau \alpha_{3}+\alpha_{4}\right)+\theta_{110}\left\{\alpha_{2}\left(\tau \gamma_{4}+\gamma_{3}\right)+\gamma_{1}\left(\tau \alpha_{3}+\alpha_{4}\right)\right\}\right. \\
& \left.\quad+2 \theta_{020} \gamma_{1}\left(\tau \gamma_{4}+\gamma_{3}\right)\right] \\
& +A_{100}^{(2)^{2}}\left[\theta_{200}\left(\tau \alpha_{3}+\alpha_{4}\right)^{2}+\theta_{110}\left(\tau \alpha_{3}+\alpha_{4}\right)\left(\tau \gamma_{4}+\gamma_{3}\right)+\theta_{020}\left(\tau \gamma_{4}+\gamma_{3}\right)^{2}\right] \\
S_{200}= & A_{100}^{(1)}\left[\bar{\theta}_{200} \alpha_{2}^{2}+\bar{\theta}_{110} \alpha_{2} \gamma_{1}+\bar{\theta}_{020} \gamma_{1}^{2}\right] \\
& +A_{100}^{(1)} A_{100}^{(2)}\left[2 \bar{\theta}_{200} \alpha_{2}\left(\tau \alpha_{3}+\alpha_{4}\right)+\bar{\theta}_{110}\left\{\alpha_{2}\left(\tau \gamma_{4}+\gamma_{3}\right)+\gamma_{1}\left(\tau \alpha_{3}+\alpha_{4}\right)\right\}\right. \\
& \left.\quad+2 \bar{\theta}_{020} \gamma_{1}\left(\tau \gamma_{4}+\gamma_{3}\right)\right] \\
& +A_{100}^{(2)^{2}}\left[\bar{\theta}_{200}\left(\tau \alpha_{3}+\alpha_{4}\right)^{2}+\bar{\theta}_{110}\left(\tau \alpha_{3}+\alpha_{4}\right)\left(\tau \gamma_{4}+\gamma_{3}\right)+\bar{\theta}_{020}\left(\tau \gamma_{4}+\gamma_{3}\right)^{2}\right] .
\end{aligned}
$$

The initial conditions are

$$
\rho_{200}=0, \quad \sigma_{200}=0, \quad \rho_{200}^{\prime}=0, \quad \sigma_{200}^{\prime}=0 .
$$


Since equations (150) are linear, and their left members have the same form as (51), the solutions will have the form

$$
\begin{aligned}
\rho_{200}=A \alpha_{2}+B \alpha_{1}+C \alpha_{3} & +D\left[\tau \alpha_{3}+\alpha_{4}\right] \\
& +A_{100}^{(1)} \phi_{1}(\tau)+A_{100}^{(1)} A_{100}^{(2)} \phi_{2}(\tau)+A_{100}^{(2)} \phi_{3}(\tau), \\
\sigma_{200}=A \gamma_{1}+B \gamma_{2}+C \gamma_{4} & +D\left[\tau \gamma_{4}+\gamma_{3}\right] \\
& +A_{100}^{(1)^{2}} \psi_{1}(\tau)+A_{100}^{(1)} A_{100}^{(2)} \psi_{2}(\tau)+A_{100}^{(2)^{2}} \psi_{3}(\tau) .
\end{aligned}
$$

Imposing the initial conditions we find

$$
\begin{aligned}
& B=C=0, \\
& A=\frac{\psi_{1}^{\prime}(0) \alpha_{4}(0)-\phi_{1}(0)\left[\gamma_{4}(0)+\gamma_{3}^{\prime}(0)\right]}{\Delta} A_{100}^{(1) 2} \\
& +\frac{\psi_{2}^{\prime}(0) \alpha_{4}(0)-\phi_{2}(0)\left[\gamma_{4}(0)+\gamma_{3}^{\prime}(0)\right]}{\Delta} A_{100}^{(1)} A_{100}^{(2)} \\
& +\frac{\psi_{3}^{\prime}(0) \alpha_{4}(0)-\phi_{3}(0)\left[\gamma_{4}(0)+\gamma_{3}^{\prime}(0)\right]}{\Delta} A_{100}^{(2)}, \\
& D=\frac{\phi_{1}(0) \gamma_{1}^{\prime}(0)-\psi_{1}^{\prime}(0) \alpha_{2}(0)}{\Delta} A_{100}^{(1)}+\frac{\phi_{2}(0) \gamma_{1}^{\prime}(0)-\psi_{2}^{\prime}(0) \alpha_{2}(0)}{\Delta} A_{100}^{(1)} A_{100}^{(2)} \\
& +\frac{\phi_{3}(0) \gamma_{1}^{\prime}(0)-\psi_{3}^{\prime}(0) \alpha_{2}(0)}{\Delta} A_{100}^{(2)^{2}} \text {. }
\end{aligned}
$$

Substituting these values in (151) we have

where

$$
\begin{aligned}
& \rho_{200}=A_{100}^{(1)} x_{1}(\tau)+A_{100}^{(1)} A_{100}^{(2)} x_{2}(\tau)+A_{100}^{(2)} x_{3}(\tau), \\
& \sigma_{200}=A_{100}^{(1)} y_{1}(\tau)+A_{100}^{(1)} A_{100}^{(2)} y_{2}(\tau)+A_{100}^{(2)^{2}} y_{3}(\tau),
\end{aligned}
$$

$x_{1}(\tau)=\phi_{1}(\tau)+\frac{\psi_{1}^{\prime}(0) \alpha_{4}(0)-\phi_{1}(0)\left[\gamma_{4}^{\prime}(0)+\gamma_{3}^{\prime}(0)\right]}{\Delta} \alpha_{2}(\tau)$

$$
+\frac{\phi_{1}(0) \gamma_{1}^{\prime}(0)-\psi_{1}^{\prime}(0) \alpha_{2}(0)}{\Delta}\left[\alpha_{4}(\tau)+\tau \alpha_{3}(\tau)\right],
$$

$y_{1}(\tau)=\psi_{1}(\tau)+\frac{\psi_{1}^{\prime}(0) \alpha_{4}(0)-\phi_{1}(0)\left[\gamma_{4}(0)+\gamma_{3}^{\prime}(0)\right]}{\Delta} \gamma_{1}(\tau)$

$$
+\frac{\phi_{1}(0) \gamma_{1}^{\prime}(0)-\psi_{1}^{\prime}(0) \alpha_{2}(0)}{\Delta}\left[\gamma_{3}(\tau)+\tau \gamma_{4}(\tau)\right]
$$

and similar expressions for $x_{2}, y_{2}$ and $x_{3}, y_{3}$, which we shall find later [equation (172)] do not interest us. The characters of $x_{1}$ and $y_{1}$ are known with the exception of $\phi_{1}$ and $\psi_{1}$ which we will now investigate. The functions $\phi_{1}$ and $\psi_{1}$ 
are those portions of the solution of the differential equations $(150)$ which depend upon the coefficients of $A_{100}^{(1)^{2}}$. These coefficients are homogeneous of the second degree in $\alpha_{2}(\tau)$ and $\gamma_{1}(\tau)$. In $R_{200}$ and $S_{200}$ the expressions

$\theta_{200}, \bar{\theta}_{110}$ and $\theta_{020}$ contain only cosines of even multiples of $\tau$;

$\bar{\theta}_{200}, \theta_{110}$ and $\bar{\theta}_{020}$ contain only sines of odd multiples of $\tau$;

$\alpha_{2}(\tau)$ has the form $\alpha_{2}=\sum_{k} a_{k} \cos [(2 k+1) \pm \lambda] \tau$;

$\gamma_{1}(\tau)$ has the form $\gamma_{1}=\sum_{k} b_{k} \sin [2 k \pm \lambda] \tau$.

Consequently, so far as the coefficients of $A_{100}^{(1) 2}$ are concerned, $R_{200}$ and $S_{200}$ have the form

$$
\begin{aligned}
& R_{200}=\left\{\sum_{k} a_{k}^{(1)} \cos 2 k t+\sum_{k} a_{k}^{(2)} \cos [2 k \pm 2 \lambda] \tau\right\} A_{100}^{(1)^{2}}+\cdots, \\
& S_{200}=\left\{\sum_{k} b_{k}^{(1)} \sin (2 k+1) \tau+\sum_{k} b_{k}^{(2)} \sin [(2 k+1) \pm 2 \lambda] \tau\right\} A_{100}^{(1)^{2}}+\cdots
\end{aligned}
$$

By Theorem II terms involving $2 \lambda$ give rise only to periodic terms in the solution whose period is $2 K \pi$. By Theorem $\mathrm{V}$ those parts of $\rho_{200}$ and $\sigma_{200}$ depending upon the terms in $R_{200}$ and $S_{200}$ which are independent of $\lambda$ have the form

$$
\rho=p_{1}(\tau)+c \tau \alpha_{3}(\tau), \quad \sigma=p_{2}(\tau)+c \tau \gamma_{4}(\tau)
$$

respectively, where $p_{1}$ and $p_{2}$ are periodic with the period $2 \pi$. Consequently the characters of $x_{1}(\tau)$ and $y_{1}(\tau)$ are

$$
x_{1}(\tau)=\wp_{1}(\tau)+c_{1} \tau \alpha_{3}(\tau), \quad y_{1}(\tau)=\wp_{2}(\tau)+c_{1} \tau \gamma_{4}(\tau),
$$

where $\wp_{1}(\tau)$ and $\wp_{2}(\tau)$ are periodic with the period $2 K \pi$.

Coefficient of $\alpha \delta$.

$$
\rho_{110}^{\prime \prime}+\theta_{2} \rho_{110}+\theta_{3} \sigma_{110}=R_{110}, \quad \sigma_{110}^{\prime \prime}+\theta_{4} \sigma_{110}+\theta_{3} \rho_{110}=S_{110}
$$
where

$$
\begin{aligned}
R_{110}= & 2 A_{100}^{(1)} A_{010}^{(1)}\left[\theta_{200} \alpha_{2}^{2}+\theta_{110} \alpha_{2} \gamma_{1}+\theta_{020} \gamma_{1}^{2}\right] \\
& +\left[A_{100}^{(1)} A_{010}^{(2)}+A_{100}^{(2)} A_{010}^{(1)}\right]\left[2 \theta_{200} \alpha_{2}\left(\tau \alpha_{3}+\alpha_{4}\right)+\theta_{110}\left\{\gamma_{1}\left(\tau \alpha_{3}+\alpha_{4}\right)+\alpha_{2}\left(\tau \gamma_{4}+\gamma_{3}\right)\right\}\right. \\
& \left.\quad+2 \theta_{020} \gamma_{1}\left(\tau \gamma_{4}+\gamma_{3}\right)\right] \\
& +2 A_{100}^{(2)} A_{010}^{(2)}\left[\theta_{200}\left(\tau \alpha_{3}+\alpha_{4}\right)^{2}+\theta_{110}\left(\tau \alpha_{3}+\alpha_{4}\right)\left(\tau \gamma_{4}+\gamma_{3}\right)+\theta_{020}\left(\tau \gamma_{4}+\gamma_{3}\right)^{2}\right], \\
S_{110}= & 2 A_{100}^{(1)} A_{010}^{(1)}\left[\bar{\theta}_{200} \alpha_{2}^{2}+\bar{\theta}_{110} \alpha_{2} \gamma_{1}+\bar{\theta}_{020} \gamma_{1}^{2}\right] \\
& +\left[A_{100}^{(1)} A_{010}^{(2)}+A_{100}^{(2)} A_{010}^{(1)}\right]\left[2 \bar{\theta}_{200} \alpha_{2}\left(\tau \alpha_{3}+\alpha_{4}\right)+\bar{\theta}_{110}\left\{\gamma_{1}\left(\tau \alpha_{3}+\alpha_{4}\right)+\alpha_{2}\left(\tau \gamma_{4}+\gamma_{3}\right)\right\}\right. \\
& \left.\quad+2 \bar{\theta}_{020} \gamma_{1}\left(\tau \gamma_{4}+\gamma_{3}\right)\right] \\
& +2 A_{100}^{(2)} A_{010}^{(2)}\left[\bar{\theta}_{200}\left(\tau \alpha_{3}+\alpha_{4}\right)^{2}+\bar{\theta}_{110}\left(\tau \alpha_{3}+\alpha_{4}\right)\left(\tau \gamma_{4}+\gamma_{3}\right)+\bar{\theta}_{020}\left(\tau \gamma_{4}+\gamma_{3}\right)^{2}\right] .
\end{aligned}
$$


The functions $R_{110}$ and $S_{110}$ differ from $R_{200}$ and $S_{200}$ only in the constants $A_{i j k}$. The initial conditions impose the same conditional equations. Consequently the solutions differ only in the constants $A_{i j k}$, so that we can express them at once without computation:

$$
\begin{aligned}
& \rho_{110}=2 A_{100}^{(1)} A_{010}^{(1)} x_{1}(\tau)+\left[A_{100}^{(1)} A_{010}^{(2)}+A_{100}^{(2)} A_{010}^{(1)}\right] x_{2}(\tau)+2 A_{100}^{(2)} A_{010}^{(2)} x_{3}(\tau), \\
& \sigma_{110}=2 A_{100}^{(1)} A_{010}^{(1)} y_{1}(\tau)+\left[A_{100}^{(1)} A_{010}^{(2)}+A_{100}^{(2)} A_{010}^{(1)}\right] y_{2}(\tau)+2 A_{100}^{(2)} A_{010}^{(2)} y_{3}(\tau),
\end{aligned}
$$

where the $x_{i}(\tau)$ and $y_{i}(\tau)$ are the same functions of $\tau$ as before.

Coefficient of $\delta^{2}$.

By symmetry with the coefficient of $\alpha^{2}$ it is seen that

$$
\begin{aligned}
& \rho_{020}=A_{010}^{(1)} x_{1}(\tau)+A_{010}^{(1)} A_{010}^{(2)} x_{2}(\tau)+A_{010}^{(2)^{2}} x_{3}(\tau), \\
& \sigma_{020}=A_{010}^{(1)} y_{1}(\tau)+A_{010}^{(1)} A_{010}^{(2)} y_{2}(\tau)+A_{010}^{(2)} y_{3}(\tau) .
\end{aligned}
$$

\section{Coefficient of $\epsilon^{2}$.}

Since the coefficients of the first powers of $\alpha$ and $\delta$ were homogeneous in the $A_{i j k}$ of the first degree, the coefficients of $\alpha^{2}$, $\alpha \delta$ and $\delta^{2}$ are homogeneous in the $A_{i j k}$ of the second degree. The coefficient of the first power of $\epsilon$ is not homogeneous in the $A_{i j l}$, hence the second power is not homogeneous. But if the functions $\alpha_{6}(\tau)$ and $\gamma_{6}(\tau)$ were zero the coefficient of the first power of $\epsilon$ would be homogeneous. By symmetry therefore, we can at once write down the terms involving the $A_{i j k}$ to the second degree. To these must be added terms in the first degree and one term independent of the $A_{i j k}$.

The differential equations are

$$
\begin{aligned}
& \rho_{002}^{\prime \prime}+\theta_{2} \rho_{002}+\theta_{3} \sigma_{002}=R_{002}, \\
& \sigma_{002}^{\prime \prime}+\theta_{4} \sigma_{002}+\theta_{3} \rho_{002}=S_{002} ; \\
& R_{002}=\theta_{200} \rho_{001}^{2}+\theta_{110} \rho_{001} \sigma_{001}+\theta_{020} \sigma_{001}^{2}+\theta_{101} \rho_{001}, \\
& S_{002}=\bar{\theta}_{200} \rho_{001}^{2}+\bar{\theta}_{110} \rho_{001} \sigma_{001}+\theta_{020} \sigma_{001}^{2} .
\end{aligned}
$$

The terms involved in these expressions are shown in the following table:

$$
R_{002}
$$

\begin{tabular}{c|c|c|c|c|c|c|c}
\hline \hline Term & $\boldsymbol{A}_{001}^{(1)^{2}}$ & $\boldsymbol{A}_{001}^{(1)} A_{001}^{(2)}$ & $A_{001}^{(2)}$ & $A_{001}^{(1)}$ & $A_{001}^{(2)}$ & 1 & $\begin{array}{c}\text { Multiplied } \\
\text { by }\end{array}$ \\
\hline$\rho_{001}^{2}$ & $a_{2}^{2}$ & $2 \alpha_{2}\left(\tau a_{3}+a_{4}\right)$ & $\left(\tau a_{3}+a_{4}\right)^{2}$ & $2 a_{2} a_{6}$ & $2 a_{6}\left(\tau a_{3}+a_{4}\right)$ & $a_{6}^{2}$ & $\theta_{200}$ \\
$\rho_{001} \sigma_{001}$ & $a_{2} \gamma_{1}$ & $\begin{array}{c}\gamma_{1}\left(\tau a_{3}+a_{4}\right) \\
+a_{2}\left(\tau \gamma_{4}+\gamma_{3}\right)\end{array}$ & $\left(\tau a_{3}+a_{4}\right)\left(\tau \gamma_{4}+\gamma_{3}\right)$ & $\begin{array}{c}\gamma_{1} \sigma_{6} \\
+a_{2} \gamma_{6}\end{array}$ & $\begin{array}{c}a_{6}\left(\tau \gamma_{4}+\gamma_{3}\right) \\
+\gamma_{6}\left(\tau a_{3}+a_{4}\right)\end{array}$ & $a_{6} \gamma_{6}$ & $\theta_{110}$ \\
$\sigma_{001}^{2}$ & $\gamma_{1}^{2}$ & $2 \gamma_{1}\left(\tau \gamma_{4}+\gamma_{3}\right)$ & $\left(\tau \gamma_{4}+\gamma_{3}\right)^{2}$ & $2 \gamma_{1} \gamma_{6}$ & $2\left(\tau \gamma_{4}+\gamma_{3}\right)$ & $\gamma_{6}^{2}$ & $\theta_{020}$ \\
$\rho_{001}$ & & & & $a_{2}$ & $\left(\tau a_{3}+a_{4}\right)$ & $a_{6}$ & $\theta_{101}$ \\
\hline
\end{tabular}


In order to obtain the $S_{002}$ it is necessary in the above table only to change the $\theta_{i j k}$ in the last column into $\bar{\theta}_{i j k}$.

The solutions of equations (157) may be expressed in the form

$$
\begin{aligned}
& \rho_{002}=A_{001}^{(1)} x_{1}(\tau)+A_{001}^{(1)} A_{001}^{(2)} x_{2}(\tau)+A_{001}^{(2)^{2}} x_{3}(\tau)+A_{001}^{(1)} x_{4}(\tau)+A_{001}^{(2)} x_{5}(\tau)+x_{6}(\tau), \\
& \sigma_{002}=A_{001}^{(1)^{2}} y_{1}(\tau)+A_{001}^{(1)} A_{001}^{(2)} y_{2}(\tau)+A_{001}^{(2)^{2}} y_{3}(\tau)+A_{001}^{(1)} y_{4}(\tau)+A_{001}^{(2)} y_{5}(\tau)+y_{6}(\tau) .
\end{aligned}
$$

The coefficients of $A_{001}^{(1)}$ in the differential equations are homogeneous of the first degree in $\alpha_{2}$ and $\gamma_{1}$, every term of which involves the first multiple of $\lambda \tau$. Hence the solutions for these terms by Theorem II involve non-periodic terms, and we can write

$$
\begin{aligned}
& x_{4}(\tau)=\wp_{3}(\tau)+c_{2} \tau \alpha_{1}(\tau)+c_{3} \tau \alpha_{3}(\tau), \\
& y_{4}(\tau)=\wp_{4}(\tau)+c_{2} \tau \gamma_{2}(\tau)+c_{3} \tau \gamma_{4}(\tau),
\end{aligned}
$$

where $\wp_{3}(\tau)$ and $\wp_{4}(\tau)$ are periodic with the period $2 K \pi$. As is seen from the table, $x_{6}(\tau)$ and $y_{6}(\tau)$ do not involve the $\lambda$. They have therefore the form

$$
\begin{aligned}
& x_{6}(\tau)=\wp_{5}(\tau)+c_{4} \tau \alpha_{3}(\tau), \\
& y_{6}(\tau)=\wp_{6}(\tau)+c_{4} \tau \gamma_{4}(\tau) .
\end{aligned}
$$

It will be verified in (172) that we do not need to know the character of $x_{5}$ and $y_{5}$. Coefficient of $\alpha \epsilon$.

The differential equations are

\begin{tabular}{|c|c|c|c|c|c|c|}
\hline \multicolumn{7}{|c|}{$R_{101}$} \\
\hline & $2 A_{100}^{(1)} A_{001}^{(1)}$ & $A_{100}^{(1)} A_{001}^{(2)}+A_{100}^{(2)} A_{001}^{(1)}$ & $2 A_{100}^{(2)} A_{001}^{(2)}$ & $A_{100}^{(1)}$ & $A_{100}^{(2)}$ & $\underset{\text { by }}{\text { Multiplied }}$ \\
\hline$\rho_{100} \rho_{001}$ & $\alpha_{2}^{2}$ & $2 \alpha_{2}\left(\tau \alpha_{3}+\alpha_{4}\right)$ & $\left(\tau \alpha_{3}+\alpha_{4}\right)^{2}$ & $2 \alpha_{2} x_{6}$ & $2 \alpha_{6}\left(\tau \alpha_{3}+\alpha_{4}\right)$ & $\theta_{200}$ \\
\hline $\begin{array}{c}\rho_{100} \sigma_{001} \\
+\rho_{001} \sigma_{100}\end{array}$ & $a_{2} \gamma_{1}$ & $\begin{array}{c}\gamma_{1}\left(\tau \alpha_{3}+\alpha_{4}\right) \\
+\alpha_{2}\left(\tau \gamma_{4}+\gamma_{3}\right)\end{array}$ & $\left(\tau \alpha_{3}+\alpha_{4}\right)\left(\tau \gamma_{4}+\gamma_{3}\right)$ & $a_{6} \gamma_{1}+a_{2} \gamma_{6}$ & $\begin{array}{l}\alpha_{6}\left(\tau \gamma_{4}+\gamma_{3}\right) \\
+\gamma_{6}\left(\tau \alpha_{3}+\alpha_{4}\right)\end{array}$ & $\theta_{110}$ \\
\hline$\sigma_{100} \sigma_{001}$ & $\gamma_{1}^{2}$ & $2 \gamma_{1}\left(\tau \gamma_{4}+\gamma_{3}\right)$ & $\left(\tau \gamma_{4}+\gamma_{3}\right)^{2}$ & $2 \gamma_{1} \gamma_{6}$ & $2 \gamma_{6}\left(\tau \gamma_{4}+\gamma_{3}\right)$ & $\theta_{020}$ \\
\hline$\rho_{100}$ & & & & $a_{2}$ & $\left(\tau \alpha_{3}+\alpha_{4}\right)$ & $\theta_{101}$ \\
\hline
\end{tabular}

$$
\begin{gathered}
\rho_{101}^{\prime \prime}+\theta_{2} \rho_{101}+\theta_{3} \sigma_{101}=R_{101}, \\
\sigma_{101}^{\prime \prime}+\theta_{4} \sigma_{101}+\theta_{3} \rho_{101}=S_{101} . \\
R_{101}=\theta_{200}\left[2 \rho_{100} \rho_{001}\right]+\theta_{110}\left[\rho_{100} \sigma_{001}+\rho_{001} \sigma_{100}\right]+\theta_{020}\left[2 \sigma_{100} \sigma_{001}\right]+\theta_{101} \rho_{100},
\end{gathered}
$$$$
S_{101}=\bar{\theta}_{200}\left[2 \rho_{100} \rho_{001}\right]+\bar{\theta}_{110}\left[\rho_{100} \sigma_{001}+\rho_{001} \sigma_{100}\right]+\bar{\theta}_{020}\left[2 \sigma_{100} \sigma_{001}\right] \text {. }
$$

The following table shows the character of the terms entering into these expressions :

In order to obtain $S_{101}$ it is necessary only to change the $\theta_{i j k}$ in the last column into $\bar{\theta}_{i j k}$. This table shows that $R_{101}$ and $S_{101}$ differ from $R_{002}$ and $S_{002}$ only in 
the constants $A_{i j k}$. Since the initial conditions impose the same conditional equations as for the coefficient of $\epsilon^{2}$ we can at once write down the solution

$$
\begin{aligned}
\rho_{101}=2 A_{100}^{(1)} A_{001}^{(1)} x_{1}(\tau)+[ & \left.A_{100}^{(1)} A_{001}^{(2)}+A_{100}^{(2)} A_{001}^{(1)}\right] x_{2}(\tau) \\
& +2 A_{100}^{(2)} A_{001}^{(2)} x_{3}(\tau)+A_{100}^{(1)} x_{4}(\tau)+A_{100}^{(2)} x_{5}(\tau), \\
\sigma_{101}=2 A_{100}^{(1)} A_{001}^{(1)} y_{1}(\tau)+[ & \left.A_{100}^{(1)} A_{001}^{(2)}+A_{100}^{(2)} A_{001}^{(1)}\right] y_{2}(\tau) \\
& +2 A_{100}^{(2)} A_{001}^{(2)} y_{3}(\tau)+A_{100}^{(1)} y_{4}(\tau)+A_{100}^{(2)} y_{5}(\tau) .
\end{aligned}
$$

Coefficient of $\delta \epsilon$.

This coefficient can be obtained by symmetry from the coefficient of $\alpha \epsilon$ by permutation of the first and second subscripts of the $A_{i j k}$. Therefore

$$
\begin{aligned}
\rho_{011}=2 A_{010}^{(1)} A_{001}^{(1)} x_{1}(\tau)+[ & \left.A_{010}^{(1)} A_{001}^{(2)}+A_{010}^{(2)} A_{001}^{(1)}\right] x_{2}(\tau) \\
& +2 A_{010}^{(2)} A_{001}^{(2)} x_{3}(\tau)+A_{010}^{(1)} x_{4}(\tau)+A_{010}^{(2)} x_{5}(\tau), \\
\sigma_{101}=2 A_{010}^{(1)} A_{001}^{(1)} y_{1}(\tau)+[ & \left.A_{010}^{(1)} A_{001}^{(2)}+A_{010}^{(2)} A_{001}^{(1)}\right] y_{2}(\tau) \\
+ & 2 A_{010}^{(2)} A_{001}^{(2)} y_{3}(\tau)+A_{010}^{(1)} y_{4}(\tau)+A_{010}^{(2)} y_{5}(\tau) .
\end{aligned}
$$

This concludes the computation of all terms up to the second order inclusive in $\alpha, \delta$ and $\epsilon$. In order to establish the existence of the periodic solutions it is not necessary to carry the computation further.

\section{§16. Existence of periodic orbits rë̈ntrant only after many revolutions.}

We have chosen the initial conditions so that at $\tau=0$ the particle is crossing the $\rho$-axis orthogonally. It is obvious geometrically that if at any future time it again crosses the $\rho$-axis perpendicularly the orbit will be a closed one and the motion in it will be periodic. The conditions that the particle shall cross the $\rho$-axis perpendicularly at $\tau=T$ is that at this epoch

$$
\rho^{\prime}=\sigma=0 \text {. }
$$

The equations of variation have the period $2 K \pi$. Therefore we shall choose $T=K \pi$. Since $\rho$ is an even series in $\tau$, and $\sigma$ is an odd series, all the purely periodic terms in $\rho^{\prime}$ and $\sigma$ are sines and consequently vanish at $\tau=K \pi$. The terms which do not vanish must carry $\tau$ as a factor. The conditions for periodicity give us two equations, namely, at $\tau=K \pi$,

$$
\begin{aligned}
\rho^{\prime}=0=a_{100} \alpha+a_{010} \delta+a_{001} \epsilon+ & a_{200} \alpha^{2}+a_{110} \alpha \delta \\
& +a_{020} \delta^{2}+a_{101} \alpha \epsilon+a_{011} \delta \epsilon+a_{002} \epsilon^{2}+\cdots, \\
\sigma=0=b_{100} \alpha+b_{010} \delta+b_{001} \epsilon+ & b_{200} \alpha^{2}+b_{110} \alpha \delta \\
& +b_{020} \delta^{2}+b_{101} \alpha \epsilon+b_{011} \delta \epsilon+b_{002} \epsilon^{2}+\cdots,
\end{aligned}
$$


where $a_{i j k}$ and $b_{i j k}$ are the values derived from the series just computed. Their values are as follows:

$$
\begin{array}{lll}
a_{100}=A_{100}^{(2)} u, & a_{010}=A_{010}^{(2)} u, & a_{001}=A_{001}^{(2)} u, \\
b_{100}=A_{100}^{(2)} v, & b_{010}=A_{010}^{(2)} v, & b_{001}=A_{001}^{(2)} v,
\end{array}
$$

$a_{200}=A_{100}^{(1)^{2}} \bar{x}_{1}+A_{100}^{(1)} A_{100}^{(2)} \bar{x}_{2}+A_{100}^{(2)^{2}} \bar{x}_{3}$,

$b_{200}=A_{100}^{(1)^{2}} \bar{y}_{1}+A_{100}^{(1)} A_{100}^{(2)} \bar{y}_{2}+A_{\mathrm{i} 00}^{(2)^{2}} \bar{y}_{3}$,

$a_{110}=2 A_{100}^{(1)} A_{010}^{(1)} \bar{x}_{1}+\left[A_{100}^{(1)} A_{010}^{(2)}+A_{100}^{(2)} A_{010}^{(1)}\right] \bar{x}_{2}+2 A_{100}^{(2)} A_{010}^{(2)} \bar{x}_{3}$,

$b_{110}=2 A_{100}^{(1)} A_{010}^{(1)} \bar{y}_{1}+\left[A_{100}^{(1)} A_{010}^{(2)}+A_{100}^{(2)} A_{010}^{(1)}\right] \bar{y}_{2}+2 A_{100}^{(2)} A_{010}^{(2)} \bar{y}_{3}$,

$a_{020}=A_{010}^{(1)} \bar{x}_{1}+A_{010}^{(1)} A_{010}^{(2)} \bar{x}_{2}+A_{010}^{(2)} \bar{x}_{3}$,

$b_{020}=A_{010}^{(1)} \bar{y}_{1}+A_{010}^{(1)} A_{010}^{(2)} \bar{y}_{2}+A_{010}^{()^{2}} \bar{y}_{3}$,

$a_{101}=2 A_{100}^{(1)} A_{001}^{(1)} \bar{x}_{1}+\left[A_{100}^{(1)} A_{001}^{(2)}+A_{100}^{(2)} A_{001}^{(1)}\right] \bar{x}_{2}+2 A_{100}^{(2)} A_{001}^{(2)} \bar{x}_{3}+A_{100}^{(1)} \bar{x}_{4}+A_{100}^{(2)} \bar{x}_{5}$, $b_{101}=2 A_{100}^{(1)} A_{001}^{(1)} \bar{y}_{1}+\left[A_{100}^{(1)} A_{001}^{(2)}+A_{100}^{(2)} A_{001}^{(1)}\right] \bar{y}_{2}+2 A_{100}^{(2)} A_{001}^{(2)} \bar{y}_{3}+A_{100}^{(1)} \bar{y}_{4}+A_{100}^{(2)} \bar{y}_{5}$,

$a_{011}=2 A_{010}^{(1)} A_{001}^{(1)} \bar{x}_{1}+\left[A_{010}^{(1)} A_{001}^{(2)}+A_{010}^{(2)} A_{001}^{(1)}\right] \bar{x}_{2}+2 A_{010}^{(2)} A_{001}^{(2)} \bar{x}_{3}+A_{010}^{(1)} \bar{x}_{4}+A_{010}^{(2)} \bar{x}_{5}$, $b_{011}=2 A_{010}^{(1)} A_{001}^{(1)} \bar{y}_{1}+\left[A_{010}^{(1)} A_{001}^{(2)}+A_{010}^{(2)} A_{001}^{(1)}\right] \bar{y}_{2}+2 A_{010}^{(2)} A_{001}^{(2)} \bar{y}_{3}+A_{010}^{(1)} \bar{y}_{4}+A_{010}^{(2)} \bar{y}_{5}$,

$a_{002}=A_{001}^{(1)^{2}} \bar{x}_{1}+A_{001}^{(1)} A_{001}^{(2)} \bar{x}_{2}+A_{001}^{(2)} \bar{x}_{3}+A_{001}^{(1)} \bar{x}_{4}+A_{001}^{(2)} \bar{x}_{5}+\bar{x}_{6}$,

$b_{002}=A_{001}^{(1)} \bar{y}_{1}+A_{001}^{(1)} A_{001}^{(2)} \bar{y}_{2}+A_{001}^{(2)^{2}} \bar{y}_{3}+A_{001}^{(1)} \bar{y}_{4}+A_{001}^{(2)} \bar{y}_{5}+\bar{y}_{6}$,

where

$$
u=K \pi \frac{d \alpha_{3}}{d \tau}, \quad v=k \pi \gamma_{4},
$$

$$
\bar{x}_{i}=\frac{d x_{i}}{d \tau}, \quad \bar{y}_{i}=y_{i},
$$

at $\tau=K \pi$.

Let us solve now the equation (164), $\rho^{\prime}=0$, for $\epsilon$ as a power series in $\alpha$ and $\delta$. We obtain

$$
\epsilon=\epsilon_{10} \alpha+\epsilon_{01} \delta+\epsilon_{20} \alpha^{2}+\epsilon_{11} \alpha \delta+\epsilon_{02} \delta^{2}+\cdots,
$$

where the coefficients $\epsilon_{i j}$ have the following values:

$$
\begin{aligned}
& \epsilon_{10}=-\frac{a_{100}}{a_{001}} \\
& \epsilon_{01}=-\frac{a_{010}}{a_{001}} \\
& \epsilon_{20}=-\frac{a_{200} a_{001}^{2}-a_{100} a_{101} a_{001}+a_{002} a_{100}^{2}}{a_{001}^{3}},
\end{aligned}
$$




$$
\begin{aligned}
& \epsilon_{11}=-\frac{a_{110} a_{001}^{2}-a_{011} a_{100} a_{001}-a_{101} a_{010} a_{001}+2 a_{002} a_{100} a_{010}}{a_{001}^{3}}, \\
& \epsilon_{02}=-\frac{a_{020} a_{001}^{2}-a_{011} a_{010} a_{001}+a_{002} a_{010}^{2}}{a_{001}^{3}}
\end{aligned}
$$

Solving now equation (164), $\sigma=0$, for $\epsilon$ in terms of $\alpha$ and $\delta$, we obtain

$$
\epsilon=\bar{\epsilon}_{10} \alpha+\bar{\epsilon}_{01} \delta+\bar{\epsilon}_{20} \alpha^{2}+\bar{\epsilon}_{11} \alpha \delta+\bar{\epsilon}_{02} \delta^{2}+\cdots,
$$

where the $\bar{\epsilon}_{i j}$ have the same expressions in the $b_{k l m}$ as the $\epsilon_{i j}$ have in the $a_{k l m}$. Subtracting (167) from (168), we have

$$
0=\left[\bar{\epsilon}_{10}-\epsilon_{10}\right] \alpha+\left[\bar{\epsilon}_{01}-\epsilon_{01}\right] \delta+\left[\bar{\epsilon}_{20}-\epsilon_{20}\right] \alpha^{2}+\left[\bar{\epsilon}_{11}-\epsilon_{11}\right] \alpha \delta+\left[\bar{\epsilon}_{02}-\epsilon_{02}\right] \delta^{2}+\cdots
$$

We must now examine the coefficients of this series.

$$
\begin{aligned}
& {\left[\bar{\epsilon}_{10}-\epsilon_{10}\right]=\frac{a_{100}}{a_{001}}-\frac{b_{100}}{b_{001}}=\frac{A_{100}^{(2)} u}{A_{001}^{(2)} u}-\frac{A_{100}^{(2)} v}{A_{001}^{(2)} v}=0,} \\
& {\left[\bar{\epsilon}_{01}-\epsilon_{10}\right]=\frac{a_{010}}{a_{001}}-\frac{b_{010}}{b_{001}}=\frac{A_{010}^{(2)} u}{A_{001}^{(2)} u}-\frac{A_{010}^{(2)} v}{A_{001}^{(2)} v}=0 .}
\end{aligned}
$$

Both of the linear terms therefore vanish. The computation of the second degree terms is somewhat more complicated. It simplifies matters somewhat if we observe that the $\bar{\epsilon}_{i j}$ have the same expressions in $v$ and the $\bar{y}_{i}$ as the $\epsilon_{i j}$ have in $u$ and $\bar{x}_{i}$. It is sufficient therefore to compute one and derive the other from it. From (165) we have

$$
-\epsilon_{20}=\frac{a_{200}}{a_{001}}-\frac{a_{100} a_{101}}{a_{001}^{2}}+\frac{a_{002} a_{100}^{2}}{a_{001}^{3}} .
$$

Substituting in these fractions the values of the $a_{i j k}$ from (165) we get

$$
\begin{aligned}
& \underset{a_{001}}{a_{200}}=\frac{1}{A_{001}^{(2)^{3}}}\left[A_{100}^{(1)^{2}} A_{001}^{(2)^{2}} \frac{\bar{x}_{1}}{u}+A_{100}^{(1)} A_{1: 0}^{(2)} A_{001}^{(2)^{2}} \frac{\bar{x}_{2}}{u}+A_{1011}^{(2)^{2}} A_{001}^{(2)} \frac{\bar{x}_{3}}{u}\right], \\
& -\frac{a_{100} a_{101}}{a_{001}^{2}}=\frac{1}{A_{001}^{(2)}}\left[-2 A_{100}^{(1)} A_{100}^{(2)} A_{001}^{(1)} A_{001}^{(2)} \frac{\bar{x}_{1}}{u}+\left[-A_{100}^{(2)^{2}} A_{001}^{(1)} A_{001}^{(2)}-A_{100}^{(1)} A_{1001}^{(2)} A_{001}^{(2)}{ }^{2}\right] \frac{\bar{x}_{2}}{u}\right. \\
& \left.-2 A_{100}^{(2)} A_{001}^{(2)^{2}} \frac{\bar{x}_{3}}{u}-A_{100}^{(1)} A_{100}^{(2)} A_{001}^{(2)} \frac{\bar{x}_{4}}{u}-A_{100}^{(2)^{2}} A_{001}^{(2)} \frac{\bar{x}_{5}}{u}\right], \\
& \frac{a_{002} a_{100}^{2}}{a_{001}^{3}}=\frac{1}{A_{001}^{(2)^{3}}}\left[A_{100}^{(2)^{2}} A_{001}^{(1)^{2}} \frac{\bar{x}_{1}}{u}+A_{100}^{(2)} A_{001}^{(1)} A_{001}^{(2)} \frac{\bar{x}_{2}}{u}+A_{100}^{(2)^{2}} A_{001}^{(2)^{2}} \frac{\bar{x}_{3}}{u}\right. \\
& \left.+A_{100}^{(2)^{2}} A_{001}^{(1)} \frac{\bar{x}_{4}}{u}+A_{100}^{(2)^{2}} A_{001}^{(2)} \frac{\bar{x}_{5}}{u} A_{100}^{(2)^{2}} \bar{x}_{6}\right] .
\end{aligned}
$$


For the sum of these three expressions, there results

$$
\begin{aligned}
-\epsilon_{20}=-\frac{1}{A_{001}^{(2)}}\left[\left[A_{100}^{(1)} A_{001}^{(2)}-A_{100}^{(2)} A_{001}^{(1)}\right]^{2} \frac{\bar{x}_{1}}{u}\right. & \\
& \left.-\left[A_{100}^{(1)} A_{001}^{(2)}-A_{100}^{(2)} A_{001}^{(1)}\right] A_{100}^{(2)} \frac{\bar{x}_{4}}{u}+A_{100}^{(2)} \frac{\bar{x}_{6}}{u}\right],
\end{aligned}
$$

the coefficients of $\bar{x}_{2} / u, \bar{x}_{3} / u$ and $\bar{x}_{5} / u$ vanishing identically. This is the reason why it was not necessary to compute $x_{2}, x_{3}$, and $x_{5}$ in (152) and (158).

Changing the $\bar{x}_{i}$ into $\bar{y}_{i}$ and $u$ into $v$ gives us $-\bar{\epsilon}_{20}$. Hence

$$
\begin{aligned}
{\left[\bar{\epsilon}_{20}-\epsilon_{20}\right]=} & \frac{1}{A_{001}^{(2)}}\left\{\left[A_{100}^{(1)} A_{001}^{(2)}-A_{100}^{(2)} A_{001}^{(1)}\right]^{2}\left[\frac{\bar{x}_{1}}{u}-\frac{\bar{y}_{1}}{v}\right]\right. \\
& \left.-A_{100}^{(2)}\left[A_{100}^{(1)} A_{001}^{(2)}-A_{100}^{(2)} A_{001}^{(1)}\right]\left[\frac{\bar{x}_{4}}{u}-\frac{\bar{y}_{4}}{v}\right]+A_{100}^{(2)^{2}}\left[\frac{\bar{x}_{6}}{u}-\frac{\bar{y}_{6}}{v}\right]\right\} .
\end{aligned}
$$

But $\left[\bar{x}_{1} / u-\bar{y}_{1} / v\right]$ and $\left[\bar{x}_{6} / u-\bar{y}_{6} / v\right]$ both vanish since

$$
\bar{x}_{1}=c_{1} u, \quad \bar{y}_{1}=c_{1} v, \quad \bar{x}_{6}=c_{4} u, \quad \bar{y}_{6}=c_{4} v,
$$

as is readily seen from (153) and (160). It is also seen on referring to (159) that $\left[\bar{x}_{4} / u-\bar{y}_{4} / v\right]$ does not vanish, but is equal to

Hence

$$
\frac{\bar{x}_{4}}{u}-\frac{\bar{y}_{4}}{v}=c_{2}\left[\frac{1}{u} \frac{d \alpha_{1}}{d \tau}-\frac{\gamma_{2}}{v}\right]_{\tau=K \pi} .
$$

$$
\left[\bar{\epsilon}_{20}-\epsilon_{20}\right]=-\frac{A_{100}^{(2)}\left[A_{100}^{(1)} A_{001}^{(2)}-A_{100}^{(2)} A_{001}^{(1)}\right]}{A_{001}^{(2) 3}}\left[\frac{\bar{x}_{4}}{u}-\frac{\bar{y}_{4}}{v}\right] .
$$

Without repeating the details of the computation we find in a similar way

$$
\begin{aligned}
& (175)\left[\bar{\epsilon}_{11}-\epsilon_{11}\right]=-\frac{A_{010}^{(2)}\left[A_{100}^{(1)} A_{001}^{(2)}-A_{100}^{(2)} A_{001}^{(1)}\right]+A_{100}^{(2)}\left[A_{010}^{(1)} A_{001}^{(2)}-A_{010}^{(2)} A_{001}^{(1)}\right]}{A_{001}^{(2)}}\left[\frac{\bar{x}_{4}}{u}-\frac{\bar{y}_{4}}{v}\right], \\
& (176)\left[\bar{\epsilon}_{02}-\epsilon_{02}\right]=-\frac{A_{010}^{(2)}\left[A_{010}^{(1)} A_{001}^{(2)}-A_{010}^{(2)} A_{001}^{(1)}\right]}{A_{001}^{(2)}}\left[\frac{\bar{x}_{4}}{u}-\frac{\bar{y}_{4}}{v}\right] .
\end{aligned}
$$

Substituting these values in (169), we find that the second degree terms in $\alpha$ and $\delta$ are factorable. Thus (169) becomes

$$
\begin{aligned}
0=\frac{1}{A_{001}^{(2)}}\left[\frac{\bar{x}_{4}}{u}-\frac{\bar{y}_{4}}{v}\right]\left[A_{100}^{(2)} \alpha+A_{010}^{(2)} \delta+\right. & \cdots]\left[\left(A_{10}^{(1)} A_{001}^{(2)}-A_{100}^{(2)} A_{001}^{(1)}\right) \alpha\right. \\
& \left.+\left(A_{010}^{(1)} A_{001}^{(2)}-A_{010}^{(2)} A_{001}^{(1)}\right) \delta+\cdots\right] .
\end{aligned}
$$

There are therefore two real solutions for $\delta$ as power series in $\alpha$. Substituting these values of $\delta$ in (168) we find the two corresponding values of $\epsilon$. In this manner we find : 


$$
\begin{gathered}
\text { First solution. } \\
\delta=-\frac{A_{100}^{(1)} A_{001}^{(2)}-A_{010}^{(2)} A_{00}^{(1)}}{A_{010}^{(1)} A_{001}^{(2)}-A_{010}^{(2)} A_{001}^{(1)}} \alpha+\cdots=\frac{\gamma_{5}^{\prime}-a \gamma_{3}^{\prime}}{\alpha_{5}-a \alpha_{4}} \alpha+\cdots=\frac{\gamma_{6}^{\prime}(0)}{\alpha_{6}(0)} \alpha+\cdots, \\
\epsilon=\frac{A_{100}^{(1)} A_{010}^{(2)}-A_{100}^{(2)} A_{010}^{(1)}}{A_{010}^{(1)} A_{001}^{(2)}-A_{010}^{(2)} A_{001}^{(1)}} \alpha+\cdots=\frac{1}{\alpha_{5}-a \alpha_{4}} \alpha+\cdots=\frac{1}{\alpha_{6}(0)} \alpha+\cdots
\end{gathered}
$$

$$
\begin{gathered}
\text { Second solution. } \\
\delta=-\frac{A_{100}^{(2)}}{A_{010}^{(2)}} \alpha+\cdots=\frac{\gamma_{1}^{\prime}(0)}{\alpha_{2}(0)} \alpha+\cdots=\gamma_{2}^{\prime}(0) \cdot \alpha+\cdots, \\
\epsilon=-\frac{A_{100}^{(2)}}{A_{001}^{(2)}} \alpha-\frac{A_{010}^{(2)}}{A_{001}^{(2)}} \delta+\cdots=0 \cdot \alpha+\cdots,
\end{gathered}
$$

where $\alpha_{6}$ and $\gamma_{6}$ are the quantities defined in (147), and $\gamma_{6}^{\prime}(0)$ is the value of $d \gamma_{6} / d \tau$ for $\tau=0$. Thus one solution for $\epsilon$ begins with the first power of $\alpha$ while the other certainly does not begin before the second, but in both solutions $\delta$ begins with the first power of $\alpha$.

\section{\$17. Construction of the solutions having the period $2 K \pi$.}

We have just proved the existence of series for $\rho, \sigma$ and $\epsilon$ proceeding in powers of the initial value of $\rho$ (let us call this value $e$ ).* The series for $\rho$ and $\sigma$ are periodic in $\tau$ with the period $2 K \pi$, and since this condition holds for all values of $e$ sufficiently small each coefficient is separately periodic. The series for $\rho$ is even in $\tau$, and the series for $\sigma$ is odd in $\tau$. These series have the form

$$
\begin{aligned}
& \rho=\rho_{1} e+\rho_{2} e^{2}+\rho_{3} e^{3}+\cdots, \\
& \sigma=\sigma_{1} e+\sigma_{2} e^{2}+\sigma_{3} e^{3}+\cdots, \\
& \epsilon=\epsilon_{1} e+\epsilon_{2} e^{2}+\epsilon_{3} e^{3}+\cdots
\end{aligned}
$$

We may substitute these series in the differential equations and integrate the coefficients of each power of $e$ step by step, and determine the constants in such a way that $\rho$ and $\sigma$ shall be periodic, and satisfy the initial conditions

$$
\rho(0)=e, \quad \sigma(0)=0, \quad \rho^{\prime}(0)=0, \quad \sigma^{\prime}(0)=\delta,
$$

where $\delta$ is a constant at present unknown but to be determined in the process.

Substituting the series (180) in the differential equations (138), we find for the coefficient of the first power of $e$

$$
\rho_{1}^{\prime \prime}+\theta_{2} \rho_{1}+\theta_{3} \sigma_{1}=\theta_{001} \epsilon_{1}, \quad \sigma_{1}^{\prime \prime}+\theta_{4} \sigma_{1}+\theta_{3} \rho_{1}=0 .
$$

* The reason for chauging $\alpha$ to $e$ is that this parameter corresponds to the eccentricity in the two-body problem. 
Since by the condition of orthogonality $\rho$ must be even in $\tau$ and $\sigma$ odd in $\tau$, the solution complying with the condition is

$$
\begin{aligned}
& \rho_{1}=A^{(1)} \alpha_{2}(\tau)+D^{(1)}\left[\tau \alpha_{3}(\tau)+\alpha_{4}(\tau)\right]+\epsilon_{1}\left[a \tau \alpha_{3}(\tau)+\alpha_{5}(\tau)\right], \\
& \sigma_{1}=A^{(1)} \gamma_{1}(\tau)+D^{(1)}\left[\tau \gamma_{4}(\tau)+\gamma_{3}(\tau)\right]+\epsilon_{1}\left[a \tau \gamma_{4}(\tau)+\gamma_{5}(\tau)\right],
\end{aligned}
$$

where $\alpha_{5}(\tau)$ contains only cosines of even multiples of $\tau$ and $\gamma_{5}(\tau)$ contains only sines of odd multiples of $\tau$.

In order that this solution shall be periodic it is necessary and sufficient that

$$
D^{(1)}=-a \epsilon_{1} \text {. }
$$

Upon imposing this condition the solution (183) becomes [see eq. (146)]

$$
\begin{aligned}
& \rho_{1}=A^{(1)} \alpha_{2}(\tau)+\epsilon_{1}\left[\alpha_{5}(\tau)-a \alpha_{4}(\tau)\right]=A^{(1)} \alpha_{2}(\tau)+\epsilon_{1} \alpha_{6}(\tau), \\
& \sigma_{1}=A^{(1)} \gamma_{1}(\tau)+\epsilon_{1}\left[\gamma_{5}(\tau)-a \gamma_{3}(\tau)\right]=A^{(1)} \gamma_{1}(\tau)+\epsilon_{1} \gamma_{6}(\tau) .
\end{aligned}
$$

It remains to impose the initial condition that $\rho_{1}=1$ at $\tau=0$. From this condition we get

$$
1=A^{(1)} \bar{\alpha}_{2}+\epsilon_{1} \bar{\alpha}_{6},
$$

where $\bar{\alpha}_{2}$ and $\bar{\alpha}_{6}$ denote the values of these functions when $\tau=0$.

Coefficient of $e^{2}$.

$$
\begin{array}{rr}
\rho_{2}^{\prime \prime}+\theta_{2} \rho_{2}+\theta_{3} \sigma_{2}=\theta_{001} \epsilon_{2}+\theta_{101} \epsilon_{1} \rho_{1}+\theta_{200} \rho_{1}^{2}+\theta_{110} \rho_{1} \sigma_{1}+\theta_{020} \sigma_{1}^{2}=R_{2}, \\
\sigma_{2}^{\prime \prime}+\theta_{4} \sigma_{2}+\theta_{3} \rho_{2}= & \bar{\theta}_{200} \rho_{1}^{2}+\bar{\theta}_{110} \rho_{1} \sigma_{1}+\bar{\theta}_{020} \sigma_{1}^{2}=S_{2} .
\end{array}
$$

Every term of $R_{2}$ and $S_{2}$ contains either $A^{(1)}, \epsilon_{1}$ or $\epsilon_{2}$ as a factor. Arranged in this manner we have the expansions

$$
\begin{aligned}
& R_{2}=A^{(1)^{2}}\left[\theta_{200} \alpha_{2}^{2}+\theta_{110} \alpha_{2} \gamma_{1}+\theta_{020} \gamma_{1}^{2}\right] \\
& +A^{(1)} \epsilon_{2}\left[2 \theta_{200} \alpha_{2} \alpha_{6}+\theta_{110}\left(\gamma_{1} \alpha_{6}+\alpha_{2} \gamma_{6}\right)+2 \theta_{020} \gamma_{1} \gamma_{6}+\theta_{101} \alpha_{2}\right] \\
& +\epsilon_{1}^{2}\left[\theta_{200} \alpha_{6}^{2}+\theta_{110} \alpha_{6} \gamma_{6}+\theta_{020} \gamma_{6}^{2}+\theta_{101} \alpha_{6}\right]+\epsilon_{2}\left[\theta_{001}\right], \\
& \begin{array}{r}
S_{2}=A^{(1)^{2}}\left[\bar{\theta}_{200} \alpha_{2}^{2}+\bar{\theta}_{110} \alpha_{2} \gamma_{1}+\bar{\theta}_{020} \gamma_{1}^{2}\right]+A^{(1)} \epsilon_{1}\left[2 \bar{\theta}_{200} \alpha_{2} \alpha_{6}+\bar{\theta}_{110}\left(\gamma_{1} \alpha_{6}+\alpha_{2} \gamma_{6}\right)+2 \bar{\theta}_{020} \gamma_{1} \gamma_{6}\right] \\
+\epsilon_{1}^{2}\left[\bar{\theta}_{200} \alpha_{6}^{2}+\bar{\theta}_{110} \alpha_{6} \gamma_{6}+\bar{\theta}_{020} \gamma_{6}^{2}\right] .
\end{array}
\end{aligned}
$$

In order to understand the character of the solution of these equations we must examine the character of the various terms. The coefficient of $A^{(1)^{2}}$ in both $R_{2}$ and $S_{2}$ is homogeneous of the second degree in $\alpha_{2}$ and $\gamma_{1}$. Its expansion therefore involves terms carrying $2 \lambda \tau$ and terms independent of $\lambda$. By Theorem II the solution for the terms in $2 \lambda \tau$ is periodic. The terms independent of $\lambda$ are cosines of even multiples of $\tau$ in $R_{2}$ and sines of odd multiples of $\tau$ in 
$S_{2}$. These terms have the same character as those in the coefficients of $\epsilon_{1}^{2}$ and $\epsilon_{2}$ and may be considered under the discussion of those terms.

The coefficients of $A^{(1)} \epsilon_{1}$ in both $R_{2}$ and $S_{2}$ are homogeneous of the first degree in $\alpha_{2}$ and $\gamma_{1}$, all terms of which carry the first multiple of $\lambda \tau$. By Theorem VII the solution for $\rho_{2}$ will carry the term $\tau \alpha_{1}(\tau)$, and for $\sigma_{2}$ the term $\tau \gamma_{2}(\tau)$. Non-periodic terms of this character do not arise elsewhere in the solution, hence, in order to avoid them, we must take either $A^{(1)}=0$ or $\epsilon_{1}=0$. If we choose $A^{(1)}=0$, then, by (185), $\epsilon_{1}$ is determined so that we must have $\epsilon_{1}=1 / \bar{\alpha}_{6}$, thus agreeing with the first solution $(178)$ of the existence proof. But if we choose $\epsilon_{1}=0$ so that by $(185) A^{(1)}=1 / \bar{\alpha}_{2}$ we are in agreement with the second solution (179) of the existence proof. We will commence by developing the first solution. This necessitates the choice $A^{(1)}=0, \epsilon_{1}=1 / \bar{\alpha}_{6}$.

\section{First solution.}

Since $A^{(1)}=0$ all terms in $R_{2}$ and $S_{2}$ which carry $\lambda \tau$ or any multiple of it vanish. There remains

$$
\begin{aligned}
& R_{2}=\epsilon_{1}^{2}\left[\theta_{200} \alpha_{6}^{2}+\theta_{110} \alpha_{6} \gamma_{6}+\theta_{020} \gamma_{6}^{2}+\theta_{101} \alpha_{6}\right]+\epsilon_{2} \theta_{001}, \\
& S_{2}=\epsilon_{1}^{2}\left[\bar{\theta}_{200} \alpha_{6}^{2}+\bar{\theta}_{110} \alpha_{6} \gamma_{6}+\bar{\theta}_{020} \gamma_{6}^{2}\right] .
\end{aligned}
$$

We have also

$$
\rho_{1}=\frac{\alpha_{6}(\tau)}{\bar{\alpha}_{6}}, \quad \sigma_{1}=\frac{\gamma_{6}(\tau)}{\bar{\alpha}_{6}}, \quad \epsilon_{1}=\frac{1}{\bar{\alpha}_{6}} .
$$

It is easy to characterize $R_{2}$ and $S_{2}$. $\quad R_{2}$ contains only cosines of even multiples of $\tau$, and $S_{2}$ contains only sines of odd multiples of $\tau$. Since $\rho_{2}$ is even in $\tau$ and $\sigma_{2}$ odd, the solution satisfying this condition is

$$
\begin{aligned}
& \rho_{2}=A^{(2)} \alpha_{2}(\tau)+D^{(2)}\left[\tau \alpha_{3}(\tau)+\alpha_{4}(\tau)\right]+\left[\eta_{2}(\tau)+a_{2} \tau \alpha_{3}(\tau)\right]+\epsilon_{2}\left[\alpha_{5}(\tau)+a \tau \alpha_{3}(\tau)\right], \\
& \sigma_{2}=A^{(2)} \gamma_{1}(\tau)+D^{(2)}\left[\tau \gamma_{4}(\tau)+\gamma_{3}(\tau)\right]+\left[\zeta_{2}(\tau)+a_{2} \tau \gamma_{4}(\tau)\right]+\epsilon_{2}\left[\gamma_{5}(\tau)+a \tau \gamma_{4}(\tau)\right] .
\end{aligned}
$$

In this solution the terms are grouped according to their origin. The first two terms are the complementary function. The third arises from the terms carrying $\epsilon_{1}^{2}$ as a factor. The fourth arises from the terms carrying $\epsilon_{2}$ as a factor. $\quad a_{2}$ is a constant depending upon the coefficients of $\epsilon_{1}^{2}$ in the differential equations. $\quad \alpha_{5}(\tau)$ and $\gamma_{5}(\tau)$ are the same functions as in the coefficient of the first power of $e$. By Theorems IV and $\mathrm{V}, \eta_{2}(\tau)$ and $\zeta_{2}(\tau)$ are periodic functions of $\tau$ with the period $2 \pi$ and so constituted that $\eta_{2}(\tau)$ contains only cosines of even multiples of $\tau$, and $\zeta_{2}(\tau)$ contains only sines of odd multiples.

In order that $\rho_{2}$ and $\sigma_{2}$ shall be periodic we must have

$$
D^{(2)}=-a_{2}-a \epsilon_{2},
$$


and this makes

$$
\begin{aligned}
& \rho_{2}=A^{(2)} \alpha_{2}(\tau)+\epsilon_{2} \alpha_{6}(\tau)+\eta_{2}(\tau)-a_{2} \alpha_{4}(\tau), \\
& \sigma_{2}=A^{(2)} \gamma_{1}(\tau)+\epsilon_{2} \gamma_{6}(\tau)+\zeta_{2}(\tau)-a_{2} \gamma_{3}(\tau) .
\end{aligned}
$$

In order that we may satisfy the initial conditions we must have $\rho_{2}(0)=0$, and this determines $\epsilon_{2}$ so that

$$
\epsilon_{2}=\frac{a_{2} \bar{\alpha}_{4}-\bar{\eta}_{2}-A^{(2)} \bar{\alpha}_{2}}{\bar{\alpha}_{6}} .
$$

$A^{(2)}$ is not yet determined, but it is obvious that it must be zero in order to satisfy the periodicity condition on the coefficient of $e^{3}$ in which the non-periodic parts arising from terms involving the first multiple of $\lambda \tau$ carry $A^{(2)}$ as a factor. Hence the only way to avoid non-periodic terms of this character is to choose $A^{(2)}=0$. Anticipating this step then we have

$$
\begin{aligned}
& \rho_{2}=\frac{a_{2} \bar{\alpha}_{4}-\bar{\eta}_{2}}{\bar{\alpha}_{6}} \alpha_{6}(\tau)+\eta_{2}(\tau)-a_{2} \alpha_{4}(\tau), \\
& \sigma_{2}=\frac{a_{2} \bar{\alpha}_{4}-\bar{\eta}_{2}}{\bar{\alpha}_{6}} \gamma_{6}(\tau)+\zeta_{2}(\tau)-a_{2} \gamma_{3}(\tau) .
\end{aligned}
$$

Hence $\rho_{2}$ contains only cosines of even multiples of $\tau$, and $\sigma_{2}$ contains only sines of odd multiples of $\tau$.

It remains only to show that this process of integration can be carried on indefinitely. Assuming that up to and including $\rho_{i-1}$ and $\sigma_{i-1}$ every $\rho_{j}$ and $\sigma_{j}$ is periodic with the period $2 \pi$ and that the $\rho_{j}$ contain only cosines of even multiples of $\tau$ and $\sigma_{j}$ only sines of odd multiples of $\tau$, except that $\rho_{i-1}$ contains the term $A^{(i-1)} \alpha_{2}(\tau)$ and $\sigma_{i-1}$ contains the term $A^{(i-1)} \gamma_{1}(\tau)$, it will be shown that the same conditions obtain for the next succeeding step. For $\rho_{i}$ and $\sigma_{i}$ we have from the differential equations (138)

$$
\begin{aligned}
& \rho_{i}^{\prime \prime}+\theta_{2} \rho_{i}+\theta_{3} \sigma_{i}=\theta_{001} \epsilon_{i}+A^{(i-1)} {\left[\theta_{101} \epsilon_{1} \alpha_{2}+2 \theta_{200} \rho_{1} \alpha_{2}\right.} \\
&\left.+\theta_{110}\left(\rho_{1} \gamma_{1}+\sigma_{1} \alpha_{2}\right)+2 \theta_{030} \sigma_{1} \gamma_{1}\right]+\Phi_{i}, \\
& \sigma_{i}^{\prime \prime}+\theta_{4} \sigma_{i}+\theta_{3} \rho_{i}=A^{(i-1)}\left[2 \bar{\theta}_{200} \rho_{1} \alpha_{2}+\bar{\theta}_{110}\left(\rho_{1} \gamma_{1}+\sigma_{1} \alpha_{2}\right)+2 \bar{\theta}_{020} \sigma_{1} \gamma_{1}\right]+\Psi_{i} .
\end{aligned}
$$

From the properties of the differential equations it is readily seen that $\Phi_{i}$ contains only known terms all of which are cosines of even multiples of $\tau$, and that $\Psi_{i}$ contains only known terms all of which are sines of odd multiples of $\tau$. The coefficients of $A^{(i-1)}$ are homogeneous of the first degree in $\alpha_{2}$ and $\gamma_{1}$, and consequently each term involves a first multiple of $\lambda \tau$. Their solution gives rise to non-periodic terms of the form $\tau \alpha_{1}(\tau)$ and $\tau \gamma_{2}(\tau)$. They carry $A^{(i-1)}$ as a 
factor, and since terms of this type arise nowhere else in the solution we can make them disappear only by putting $A^{(i-1)}=0$. The solution for (192) then has the form

$$
\begin{aligned}
& \rho_{i}=A^{(i)} \alpha_{2}(\tau)+D^{(i)}\left[\tau \alpha_{3}(\tau)+\alpha_{4}(\tau)\right]+\left[\eta_{i}(\tau)+a_{i} \tau \alpha_{3}(\tau)\right]+\epsilon_{i}\left[\alpha_{5}(\tau)+a \tau \alpha_{3}(\tau)\right], \\
& \sigma_{i}=A^{(i)} \gamma_{1}(\tau)+D^{(i)}\left[\tau \gamma_{4}(\tau)+\gamma_{3}(\tau)\right]+\left[\zeta_{i}(\tau)+a_{i} \tau \gamma_{4}(\tau)\right]+\epsilon_{i}\left[\gamma_{5}(\tau)+a \tau \gamma_{4}(\tau)\right],
\end{aligned}
$$

where $\eta_{i}(\tau)$ and $\zeta_{i}(\tau)$ are periodic with the period $2 \pi$, and by Theorem $V, \eta_{i}(\tau)$ contains only cosines of even multiples of $\tau$, and $\zeta_{i}(\tau)$ contains only sines of odd multiples of $\tau$.

For $\rho_{i}$ and $\sigma_{i}$ to be periodic it is necessary and sufficient that

which makes

$$
D^{(i)}=-a_{i} \cdot a \epsilon_{i}
$$

$$
\begin{aligned}
& \rho_{i}=A^{(i)} \alpha_{2}(\tau)+\eta_{i}(\tau)-a_{i} \alpha_{4}(\tau)+\epsilon_{i} \alpha_{6}(\tau), \\
& \sigma_{i}=A^{(i)} \gamma_{1}(\tau)+\zeta_{i}(\tau)-a_{i} \gamma_{3}(\tau)+\epsilon_{i} \gamma_{6}(\tau) .
\end{aligned}
$$

From the initial conditions we must have $\rho_{i}(0)=0$. This condition determines $\epsilon_{i}$ to be

$$
\epsilon_{i}=\frac{a_{i} \bar{\alpha}_{4}-\bar{\eta}_{i}-A^{(i)} \bar{\alpha}_{2}}{\bar{\alpha}_{6}}
$$

Thus the constants are uniquely determined. The $\rho_{i}$ and $\sigma_{i}$ have the properties assumed for those having lower subscripts, and the process of integration can be continued indefinitely. Every $A^{(j)}$ is zero. Since no terms involving the $\lambda \tau$ enter, the solution has the period $2 \pi$. But the orbits represented belong to the class of generating orbits from which we started. In other words, we set out with a generating orbit for which the initial distance was, let us say, $r_{0}$, and we have found another generating orbit for which the initial distance is $r_{0}+e$ ( $e$ arbitrary). There is nothing surprising in this, for $r_{0}$ is a function of an arbitrary constant $\beta$. Let us suppose we had started with a definite value of $\beta$, let us say $\beta_{0}$. This gives us a definite generating orbit with a definite initial distance $r_{0}$. Let us seek now the generating orbit for which the initial distance is $r_{0}+e$. If $e$ is sufficiently small we can evidently give an increment $\epsilon$ to $\beta$ which will increase $r_{0}$ by the amount $e$. We have

$$
\begin{gathered}
r_{0}=f\left(\beta_{0}\right), \\
r_{0}+e=f\left(\beta_{0}+\epsilon\right) .
\end{gathered}
$$

Expanding the right member of the last equation by Taylor's theorem we have

$$
e=\frac{\partial f}{\partial \beta_{0}} \epsilon+\frac{1}{2} \frac{\partial^{2} f}{\partial \beta_{0}^{2}} \epsilon^{2}+\cdots
$$


and by inversion, since

which we might write

$$
\begin{gathered}
\left.\frac{\partial f}{\partial \beta}\right|_{\beta=\beta_{0}} \neq 0, \\
\epsilon=\frac{1}{\frac{\partial f}{\partial \beta_{0}}} e+\cdots,
\end{gathered}
$$

$$
\epsilon=e_{1} e+e_{2} e^{2}+\cdots
$$

Then by substituting in the generating orbit

$$
\beta=\beta_{0}+e_{1} e+e_{2} e^{2}+\cdots
$$

and arranging as a power series in $e$, we would obtain the generating orbit which we sought. As these are the same conditions that are imposed when we seek new orbits through the equations of variation it is to be anticipated that one of the class of generating orbits satisfies the conditions.

\section{Second solution.}

We return now to equation (186) where the two methods of satisfying the periodicity condition presented themselves, and we shall continue with the second solution. We choose $\epsilon_{1}=0$, which we found determines $A^{(1)}$ to be $A^{(1)}=1 / \bar{\alpha}_{2}$. From (82) it is seen that $\bar{\alpha}_{2}=\alpha_{2}(0)=1$, and therefore $A^{(1)}=1$. Hence in the second solution

$$
\rho_{1}=\alpha_{2}(\tau), \quad \sigma_{1}=\gamma_{1}(\tau) .
$$

Using these values of $A^{(1)}$ and $\epsilon_{1}, R_{2}$ and $S_{2}$ of (186) become

$$
\begin{aligned}
& R_{2}=\left[\theta_{200} \alpha_{2}^{2}+\theta_{110} \alpha_{2} \gamma_{1}+\theta_{020} \gamma_{1}^{2}\right]+\epsilon_{2} \theta_{001}, \\
& S_{2}=\left[\bar{\theta}_{200} \alpha_{2}^{2}+\bar{\theta}_{101} \alpha_{2} \gamma_{1}+\bar{\theta}_{020} \gamma_{1}^{2}\right] .
\end{aligned}
$$

All of the terms in these expressions except $\epsilon_{2} \theta_{001}$ are of the second degree in $\alpha_{2}$ and $\gamma_{1}$. Therefore they involve terms carrying $2 \lambda \tau$ and terms independent of $\lambda \tau$. $\theta_{001}$ also is independent of $\lambda \tau$. In the solution the terms depending upon $2 \lambda \tau$ are periodic by Theorem II. As for the terms independent of $\lambda, R_{2}$ contains only cosines of even multiples of $\tau$ and $S_{2}$ contains only sines of odd multiples of $\tau$. These terms give rise to non-periodic terms in the solution which has the form

$$
\begin{array}{r}
\rho_{2}=A^{(2)} \alpha_{2}(\tau)+D^{(2)}\left[\tau \alpha_{3}(\tau)+\alpha_{4}(\tau)\right]+\phi_{2}(\lambda, \tau) \\
+\left[\eta_{2}(\tau)+a_{2} \tau \alpha_{3}(\tau)\right]+\epsilon_{2}\left[\alpha_{5}(\tau)+a \tau \alpha_{3}(\tau)\right] \\
\begin{aligned}
\sigma_{2}=A^{(2)} \gamma_{1}(\tau)+D^{(2)}\left[\tau \gamma_{4}(\tau)+\gamma_{3}(\tau)\right]+\psi_{2}(\lambda, \tau) \\
+\left[\zeta_{2}(\tau)+a_{2} \tau \gamma_{4}(\tau)\right]+\epsilon_{2}\left[\gamma_{5}(\tau)+a \tau \gamma_{4}(\tau)\right]
\end{aligned}
\end{array}
$$


In these equations $\phi_{2}(\lambda, \tau)$ and $\psi_{2}(\lambda, \tau)$ are the periodic terms involving $\lambda$, $\eta_{2}$ and $\zeta_{2}$ are the periodic terms with the period $2 \pi ; a_{2}$ the constant belonging to the non-periodic part, and the coefficients of $\epsilon_{2}$ are the solutions arising from the coefficient of $\epsilon_{2}$ in the differential equations. In order that this solution (197) shall be periodic it is necessary that

and this reduces $\rho_{2}$ and $\sigma_{2}$ to

$$
D^{(2)}=-a_{2}-a \epsilon_{2},
$$

$$
\begin{aligned}
& \rho_{2}=A^{(2)} \alpha_{2}(\tau)+\phi_{2}(\lambda, \tau)+\eta_{2}(\tau)-a_{2} \alpha_{4}(\tau)+\epsilon_{2} \alpha_{6}(\tau), \\
& \sigma_{2}=A^{(2)} \gamma_{1}(\tau)+\psi_{2}(\lambda, \tau)+\zeta_{2}(\tau)-a_{2} \gamma_{3}(\tau)+\epsilon_{2} \gamma_{6}(\tau) .
\end{aligned}
$$

In order that we may satisfy the initial conditions we must have $\rho_{2}(0)=0$. Hence, since $\alpha_{2}(0)=1$,

$$
A^{(2)}=-\bar{\phi}_{2}-\bar{\eta}_{2}+a_{2} \bar{\alpha}_{4}-\epsilon_{2} \bar{\alpha}_{6} .
$$

The constant $\epsilon_{2}$ is determined by the periodicity condition for the coefficient of $e^{3}$.

Coefficient of $e^{3}$.

$$
\rho_{3}^{\prime \prime}+\theta_{2} \rho_{3}+\theta_{3} \sigma_{3}=R_{3}, \quad \sigma_{3}^{\prime \prime}+\theta_{4} \sigma_{3}+\theta_{3} \rho_{3}=S_{3},
$$

where

$$
\begin{gathered}
R_{3}=\theta_{001} \epsilon_{3}+\theta_{101} \epsilon_{2} \rho_{1}+2 \theta_{200} \rho_{1} \rho_{2}+\theta_{110}\left[\sigma_{2} \rho_{1}+\sigma_{1} \rho_{2}\right]+2 \theta_{020} \sigma_{1} \sigma_{2}+\theta_{300} \rho_{1}^{3} \\
+\theta_{210} \rho_{1}^{2} \sigma_{1}+\theta_{120} \rho_{1} \sigma_{1}^{2}+\theta_{030} \sigma_{1}^{3}, \\
S_{3} \bar{\theta}_{200} \rho_{1} \rho_{2}+\bar{\theta}_{110}\left[\sigma_{2} \rho_{1}+\sigma_{1} \rho_{2}\right]+2 \bar{\theta}_{020} \sigma_{1} \sigma_{2}+\bar{\theta}_{300} \rho_{1}^{3} \\
+\bar{\theta}_{210} \rho_{1}^{2} \sigma_{1}+\bar{\theta}_{120} \rho_{1} \sigma_{1}^{2}+\bar{\theta}_{030} \sigma_{1}^{3} .
\end{gathered}
$$

In classifying the terms which belong to the expansion of $R_{3}$ and $S_{3}$ we bear in mind :

First. The $\theta_{i j k}$ in $R_{3}$ involve only cosines of even multiples of $\tau$, except those which are coefficients of odd powers of $\sigma$ (i. e., $j$ is odd). These involve only sines of odd multiples. The reverse is the case in the $\bar{\theta}_{i j k}$ of $S_{3}$. If $j$ is even, $\bar{\theta}_{i j k}$ involves only sines of odd multiples of $\tau$. If $j$ is odd, $\hat{\theta}_{i j k}$ involve only cosines of even multiples of $\tau$.

Second. $\rho_{1}$ and $\rho_{2}$ are cosine series. The terms independent of $\lambda$ involve only even multiples of $\tau$.

$\sigma_{1}$ and $\sigma_{2}$ are sine series. The terms independent of $\lambda$ involve only odd multiples of $\tau$.

It is seen then that among the terms in $R_{3}$ which are independent of $\lambda$ only cosines of even multiples of $\tau$ enter; and among the terms in $S_{3}$ which are independent of $\lambda$ only sines of odd multiples enter. In the process of integration 
therefore two types of non-periodic terms arise, first, those coming from the terms which involve the first multiple of $\lambda \tau$, and second, those coming from the terms independent of $\lambda$. It is important therefore to separate the various terms into three classes :

(a) terms independent of $\lambda$,

(b) terms involving first multiple of $\lambda \tau$ only,

(c) terms involving multiples of $\lambda \tau$ higher than the first.

The solution for these last terms is periodic. We rewrite then the differential equations (199) as follows:

$$
\begin{array}{ccc}
\rho_{3}^{\prime \prime}+\theta_{2} \rho_{3}+\theta_{3} \sigma_{3} & =\epsilon_{3} \theta_{0011}+\epsilon_{2} f_{1}(\lambda, \tau)+f_{2}(\lambda, \tau)+f_{3}(\tau)+f_{4}(\kappa \lambda, \tau), \\
\sigma_{33}^{\prime \prime}+\theta_{4} \sigma_{3}+\theta_{3} \rho_{3} & =\quad \epsilon_{2} g_{1}(\lambda, \tau)+g_{2}(\lambda, \tau)+g_{3}(\tau)+g_{4}(\kappa \lambda, \tau) .
\end{array}
$$

The coefficients of $\epsilon_{2}$ are, explicitly,

$$
\begin{array}{ll}
f_{1}(\lambda, \tau) & =\theta_{101} \alpha_{2}+2 \theta_{200} \alpha_{2} \alpha_{6}+\theta_{110}\left(\alpha_{2} \gamma_{6}+\gamma_{1} \alpha_{6}\right)+2 \theta_{020} \gamma_{1} \gamma_{6}, \\
g_{1}(\lambda, \tau) & =\quad 2 \bar{\theta}_{200} \alpha_{2} \alpha_{6}+\bar{\theta}_{110}\left(\alpha_{2} \gamma_{6}+\gamma_{1} \alpha_{6}\right)+2 \bar{\theta}_{020} \gamma_{1} \gamma_{6} .
\end{array}
$$

These terms are homogeneous in the first degree in $\alpha_{2}$ and $\gamma_{1}$, and consequently involve only terms which carry the first multiple of $\lambda \tau$. They are of importance since they carry the undetermined constant $\epsilon_{2}$ as a factor. The solution for these terms has, by Theorem II, the form

$$
\rho=F_{1}(\lambda, \tau)+b_{1} \tau \alpha_{1}(\tau), \quad \sigma=G_{1}(\lambda, \tau)+b_{1} \tau \gamma_{2}(\tau),
$$

where $F_{1}(\lambda, \tau)$ and $G_{1}(\lambda, \tau)$ are periodic and involve only terms carrying the first multiple of $\lambda \tau$. $\quad b_{1}$ is a constant depending upon $f_{1}(\lambda, \tau)$ and $g_{1}(\lambda, \tau)$. It is found, by a calculation not difficult, to have the value

$$
b_{1}=3+\mu^{2} \wp\left(\mu^{2}\right) .
$$

The functions $f_{2}(\lambda, \tau)$ and $g_{2}(\lambda, \tau)$ have the same characters as $f_{1}(\lambda, \tau)$ and $g_{1}(\lambda, \tau)$. They are considered separately since they are independent of $\epsilon_{2}$. Their solutions may be written

$$
\rho=F_{2}(\lambda, \tau)+b_{2} \tau \alpha_{1}(\tau), \quad \sigma=G_{2}(\lambda, \tau)+b_{2} \tau \gamma_{2}(\tau) .
$$

The terms $f_{3}(\tau)$ and $g_{3}(\tau)$ are independent of $\lambda$. $f_{3}(\tau)$ contains only cosines of even multiples of $\tau$, while $g_{3}(\tau)$ contains only sines of odd multiples of $\tau$. The solution for these terms has the form

$$
\rho=F_{3}(\tau)+b_{3} \tau \alpha_{3}(\tau), \quad \sigma=G_{3}(\tau)+b_{3} \tau \gamma_{4}(\tau) .
$$

Finally, $f_{4}(k \lambda, \tau)$ and $g_{4}(k \lambda, \tau)$ involve only terms which carry multiples of $\lambda \tau$ higher than the first. The solution for these terms is

$$
\rho=F_{4}(k \lambda, \tau), \quad \sigma=G_{4}(k \lambda, \tau) .
$$


The entire solution is then

(201)

$$
\begin{aligned}
& \rho_{3}=A^{(3)} \alpha_{2}(\tau)+D^{(3)}\left[\tau \alpha_{3}(\tau)+\alpha_{4}(\tau)\right]+\epsilon_{3}\left[\alpha_{5}(\tau)+a \tau \alpha_{3}(\tau)\right] \\
&+\epsilon_{2}\left[F_{1}(\lambda, \tau)+b_{1} \tau \alpha_{1}(\tau)\right]+\left[F_{2}(\lambda, \tau)+b_{2} \tau \alpha_{1}(\tau)\right] \\
&+\left[F_{3}(\tau)+b_{3} \tau \alpha_{3}(\tau)\right]+F_{4}(k \lambda, \tau), \\
& \sigma_{3}=A^{(3)} \gamma_{1}(\tau)+D^{(3)}\left[\tau \gamma_{4}(\tau)+\gamma_{3}(\tau)\right]+\epsilon_{3}\left[\gamma_{5}(\tau)+a \tau \gamma_{4}(\tau)\right] \\
&+\epsilon_{2}\left[G_{1}(\lambda, \tau)+b_{1} \tau \gamma_{2}(\tau)\right]+\left[G_{2}(\lambda, \tau)+b_{2} \tau \gamma_{2}(\tau)\right] \\
&+\left[G_{3}(\tau)+b_{3} \tau \gamma_{4}(\tau)\right]+G_{4}(k \lambda, \tau) .
\end{aligned}
$$

All of the functions $\alpha_{i}(\tau), \gamma_{i}(\tau), F_{i}(\tau)$, and $G_{i}(\tau)$ are periodic. In order that $\rho_{3}$ and $\sigma_{3}$ shall be periodic it is necessary and sufficient that the coefficient of $\tau \alpha_{3}(\tau)$ and $\tau \gamma_{3}(\tau)$, and the coefficient of $\tau \alpha_{1}(\tau)$ and $\tau \gamma_{2}(\tau)$ be zero. That is,

and

$$
D^{(3)}=-b_{3}-a \epsilon_{3},
$$

$$
\epsilon_{2}=-\frac{b_{2}}{b_{1}}
$$

by which condition the value of $\epsilon_{2}$ is determined. In order to satisfy the initial conditions we must have $\rho_{3}=0$ at $\tau=0$, and this determines $A^{(3)}$,

$$
A^{(3)}=b_{3} \alpha_{4}(0)-\epsilon_{3} \alpha_{6}(0)+\frac{b_{2}}{b_{1}} F_{1}(0)-F_{2}(0)-F_{3}(0)-F_{4}(0) \text {. }
$$

Thus all the constants are determined except $\epsilon_{3}$, and the solution is

$$
\begin{aligned}
& \rho_{3}=A^{(3)} \alpha_{2}(\tau)-b_{3} \alpha_{4}(\tau)+\epsilon_{3} \alpha_{6}(\tau)-\frac{b_{2}}{b_{1}} F_{1}(\lambda, \tau)+F_{2}(\lambda, \tau)+F_{3}(\tau)+F_{4}(k \lambda, \tau), \\
& \sigma_{3}=A^{(3)} \gamma_{1}(\tau)-b_{3} \gamma_{3}(\tau)+\epsilon_{3} \gamma_{6}(\tau)-\frac{b_{2}}{b_{1}} G_{1}(\lambda, \tau)+G_{2}(\lambda, \tau)+G_{3}(\tau)+G_{4}(k \lambda, \tau) .
\end{aligned}
$$

The constant $\epsilon_{3}$ will be determined in satisfying the periodicity condition for the coefficient of $e^{4}$. This process of integration can be continued indefinitely. $\rho_{3}$ and $\sigma_{3}$ have the same properties that have been stated for $\rho_{2}$ and $\sigma_{2}$. It is evident from the properties of the differential equations that these properties persist for $\rho_{4}$ and $\sigma_{4}$, and so on indefinitely. The coefficient for $\epsilon_{i-1}$ in so far as it carries the first multiples of $\lambda \tau$ is always the same as for $\epsilon_{2}$. Therefore the constant $\epsilon_{i-1}$ can always be determined so as to avoid non-periodic terms of the type $\tau \alpha_{1}(\tau)$ and $\tau \gamma_{2}(\tau)$. The constant $D^{(i)}$ of integration can always be determined so as to destroy non-periodic terms of the type $\tau \alpha_{3}(\tau)$ and $\tau \gamma_{4}(\tau)$. The constant $A^{(i)}$ can always be determined so as to satisfy the initial conditions. The analysis of the type of terms entering is the same as for the subscript 3. 
We have therefore a periodic solution with the period $2 K \pi$ which is different from the class of generating orbits from which we set out, for the particle makes many revolutions before its orbit reënters. After integrating the equation $d v / d \tau=c / r^{2}$ the solution will contain five arbitrary constants (subject to the condition that $\lambda$ must be rational), corresponding to mean distance, eccentricity, inclination, node, and epoch.

University of Chicago, April 20, 1909. 